\title{
Models of Function Type for Commutative Symmetric Operator Families in Krein Spaces
}

\author{
Vladimir Strauss \\ Departmento de Matemáticas Puras y Aplicadas, Universidad Simón Bolívar, Apartado 89.000,
} Caracas 1080-A, Venezuela

Correspondence should be addressed to Vladimir Strauss, str@usb.ve

Received 26 April 2007; Revised 18 July 2007; Accepted 20 August 2007

Recommended by Stephen Clark

Commutative symmetric operator families of the so-called $D_{\mathcal{K}}^{+}$-class are considered in Krein spaces. It is proved that the restriction of a family of this type on a special kind of invariant subspace is similar to a family of operators adjoint to multiplication operators by scalar functions acting on a suitable function space.

Copyright (C) 2008 Vladimir Strauss. This is an open access article distributed under the Creative Commons Attribution License, which permits unrestricted use, distribution, and reproduction in any medium, provided the original work is properly cited.

\section{Introduction}

In the well-known monograph of Colojoară and Foiaş [1], it is mentioned (in Chapter 5, Corollary 5.7) that $J$-unitary and $J$-self-adjoint operators in Pontrjagin spaces give examples of generalized spectral operators. Actually, it is clear that this observation can be applied also to many operator classes in Krein spaces, so Operator Theory in Krein spaces is a good laboratory where different methods of general Operator Theory can be tested. One of these methods is related with model spaces of function type.

The main goal of the paper is a problem of model representation for a commutative operator family $\mathfrak{Y}$ acting on a separable Krein space and possessing a maximal nonnegative invariant subspace, presented as a direct sum of a neutral subspace with a finite dimension and a uniformly positive subspace. As it is known (see [2]), this family generates a spectral function $E_{\lambda}$ with a peculiar spectral set $\Lambda$ that provides a resolution of the spectral type for the family. In particular, with every operator $A$, one can associate a scalar function $f_{A}(\lambda)$ such that

$$
A E(\Delta)=\int_{\Delta} f_{A}(\lambda) d E_{\lambda}
$$

where $\Delta$ runs through the set of all closed intervals of the real line disjoint with $\Lambda$. 
The key results (Theorems 6.5, 6.19, and 6.29) say that there is a suitable function space (so-called basic model space) where $f_{A}(\lambda)$ generates a multiplication operator similar to a compression of $A$ on a subspace calculated through $E_{\lambda}$.

Section 2 gives a draft of problems considered in the paper, analyzing a simple case of self-adjoint operator in Pontryagin space. Section 3 contains definitions and well-known results used throughout the paper. Section 4 deals with a model representation of a resolution of the identity that is simultaneously $J$-orthogonal and similar to an orthogonal resolution of the identity. In Section 5 a notion of unbounded elements conformed with a resolution of the identity is introduced and studied. Roughly speaking, a resolution sets a correspondence between a Hilbert space and a (vector-valued) function space $L_{\vec{\sigma}}^{2}$. From this point of view, the unbounded elements correspond to measurable vector-valued functions outside of $L_{\vec{\sigma}}^{2}$. The main results are presented in Section 6, where the notion of a basic model space is introduced. Also, a relation is established between multiplication operators by scalar functions acting in a basic model space, and operators of a commutative $J$-symmetric algebra of $D_{\kappa}^{+}$-class. Here, emphasis is made on the problem of uniqueness of a basic model space for such algebras. Section 6.2 corresponds to resolutions of the identity with properties like those in Pontryagin spaces. Section 6.3 deals with general $J$-orthogonal resolutions of the identity of $D_{\mathcal{K}}^{+}$-class, and Section 6.4 contains a theorem on a model representation of a commutative $J$-symmetric operator family of $D_{\kappa}^{+}$-class. Historical and bibliographical remarks are presented in the last section.

It is assumed that the reader is familiar with elements of Krein space geometry and Operator theory (see [3-7]). In this paper, the terminology given in [8] will be used.

\section{An elementary description of the problem}

Let $\mathscr{t}$ be a separable Hilbert space with a scalar product $(\cdot, \cdot)$. $\mathscr{t}$ is said to be an indefinite metric space if it is equipped by a sesquilinear continuous Hermitian form (indefinite inner product) $[\cdot, \cdot]$ such that the corresponding quadratic form has an indefinite sign (i.e., $[x, x]$ takes positive, negative, and zero values). The indefinite inner product can be represented in the form $[\cdot, \cdot]=(G \cdot, \cdot)$, where $G$ is a so-called Gram operator. The operator $G$ is bounded and self-adjoint. If the Gram operator for an indefinite metric space is boundedly invertible and its invariant subspace that corresponds to the negative spectrum (or, alternatively, to the positive spectrum) of $G$ is finite dimensional, the space is called a Pontryagin space. In this section, we consider Pontryagin spaces only.

Now, let $A$ : $\mathscr{t} \mapsto \mathscr{t}$ be a bounded linear operator. This operator is said to be $\pi$-selfadjoint ( $\pi$-s.a.) if $[A x, y]=[x, A y]$ for all $x, y \in \mathscr{t}$. The properties of $\pi$-s.a. operators differ from the properties of ordinary self-adjoint operators. For instance, a $\pi$-s.a. can have a nonreal spectrum or/and a nontrivial Jordan chain. If $A$ has only a real spectrum, then there is, on $\mathbb{R}$, the eigen spectral function (e.s.f.) $E_{\lambda}$ of $A$ with a finite set $\Lambda$ of critical points such that $(\Delta=(a ; b), a, b \notin \Lambda)$

(a) for every $\lambda \in \mathbb{R} \backslash \Lambda$ it holds that $A E_{\lambda}=E_{\lambda} A$;

(b) $\sigma\left(\left.A\right|_{E(\Delta) \mathscr{l}}\right) \subset \bar{\Delta}$;

(c) if $\Delta \cap \Lambda=\varnothing$ then the subspace $E(\Delta) \mathscr{H}$ is positive;

(d) if $\Delta \cap \Lambda \neq \varnothing$ then the subspace $E(\Delta) \mathscr{d}$ is negative or indefinite. 
Let $\lambda_{0} \in \Lambda$. Then for every point of $\Lambda$ there are three options.

(1) For an interval $\Delta=(a ; b)$ such that $\Delta \cap \Lambda=\left\{\lambda_{0}\right\}$ and $a, b \notin \Lambda$, the spectral function $\left.E_{\lambda}\right|_{E(\Delta) \mathscr{t}}$ is bounded and the representation $\int_{\Delta} \xi E(d \xi)$ takes place.

(2) For an interval $\Delta=(a ; b)$ such that $\Delta \cap \Lambda=\left\{\lambda_{0}\right\}$ and $a, b \notin \Lambda$, the spectral function $\left.E_{\lambda}\right|_{E(\Delta) \mathscr{l}}$ is bounded but the representation $\int_{\Delta} \xi E(d \xi)$ does not take place.

(3) For an interval $\Delta=(a ; b)$ such that $\Delta \cap \Lambda=\left\{\lambda_{0}\right\}$ and $a, b \notin \Lambda$, the spectral function $\left.E_{\lambda}\right|_{E(\Delta)} \mathscr{t}$ is unbounded.

The first item means that the operator $\left.A\right|_{E(\Delta) \mathfrak{d}}$ is similar to a self-adjoint operator, that is, it is a scalar spectral operator. The second item corresponds to a spectral operator with a nontrivial finite-dimensional nilpotent summand. Thus only the third item represents a situation that is out of the ordinary theory of model representation for spectral operators with real spectrum. Below, we consider this exceptional case. Let us give an example of the case in discussion.

Example 2.1. Let the system $h, g, e_{1}, e_{2}, e_{3}, \ldots$ be an orthonormalized basis in a Hilbert space $\mathscr{d}$ and let $[x, y]=(x, h)(g, y)+(x, g)(h, y)+\sum_{j=1}^{\infty}\left(x, e_{j}\right)\left(e_{j}, y\right)$. Then this space is a Pontryagin space with $\mathcal{\kappa}=1$. Let an operator $A$ be given by the conditions

(i) $A h=0$,

(ii) $A e_{j}=1 / j \cdot\left(e_{j}+h\right), j=1,2, \ldots$,

(iii) $A g=h+\sum_{j=1}^{\infty}(1 / j) \cdot e_{j}$.

These conditions define a bounded operator, so $A$ is naturally defined on $\mathscr{d}$ and, moreover, is a $\pi$-self-adjoint operator. The spectral function of $A$ can be described by the following conditions:

$$
E\left(\left\{\frac{1}{j}\right\}\right) x=\left[x,\left(e_{j}+h\right)\right] \cdot\left(e_{j}+h\right)
$$

so

$$
E\left(\left\{\frac{1}{j}\right\}_{j=1}^{n}\right) x=\sum_{j=1}^{n}\left[x,\left(e_{j}+h\right)\right] \cdot\left(e_{j}+h\right)
$$

Put $x_{n}=1 / n \cdot \sum_{j=1}^{n} e_{j}$. Then $E\left(\{1 / j\}_{j=1}^{n}\right) x_{n}=1 / n \cdot \sum_{j=1}^{n}\left(e_{j}+h\right)=h+x_{n}$. At the same time $\left\|x_{n}\right\|=1 / \sqrt{n} \rightarrow 0$ for $n \rightarrow \infty$. Thus, for $A$ and its spectral function $E_{\curlywedge}$, we have the following results:

(i) $E_{\curlywedge}$ is unbounded;

(ii) $h$ belongs to the closure of the linear span $\widetilde{\mathscr{H}}$ generated by the subspaces $E(\Delta) \mathscr{d}$, where $\Delta=(a ; b), 0<a<b$, so the kernel of the operator $\left.A\right|_{\widetilde{d}}$ is not trivial;

(iii) $\widetilde{\mathscr{A}}$ is degenerated, $\widetilde{\mathscr{H}} \cap \widetilde{\mathscr{l}}^{\perp]}=\{\mu h\}_{\mu \in \mathbb{C}}$.

Let us note that the coexistence of these properties reflects a general situation (in particular, the first item yields the other two). Indeed, it is shown by the following theorem. 
Theorem 2.2 (see [8, Theorem I.9.6]). Let a subspace $\mathcal{\perp}$ be positive. Then there is a constant $c>1$ such that $(x, x) \leq c[x, x]$ for every $x \in \mathcal{L}$.

For simplicity everywhere below in this section, we assume that

(a) $\Lambda=\{0\}$,

(b) $E_{\curlywedge}$ is unbounded,

(c) for every interval $\Delta=[a ; b]$, such that $0 \notin \Delta$ the operator $\left.A\right|_{E(\Delta) \mathfrak{l}}$ has simple spectrum.

Then by Theorem 2.2, the subspace $\widetilde{\mathscr{H}}$ generated as the closed linear span of the subspaces $E(\Delta) \mathscr{d}$, where $\Delta=[a ; b], 0 \notin \Delta$, is not a positive subspace, thus it has the nontrivial isotropic

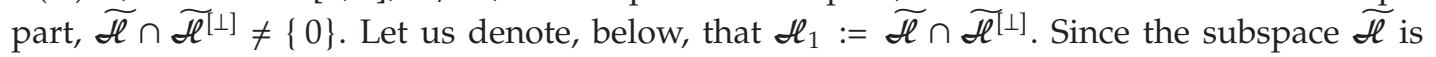
evidently nonnegative, it can be presented in the form

$$
\widetilde{d}=\mathscr{H}_{1}+\mathscr{H}_{2}
$$

where $\mathscr{t}_{2}$ is a positive subspace. Note that $\mathscr{L}_{2}$ is not uniquely defined. Note also that, due to the general theory, the subspace $\widetilde{\mathscr{d}}$ is a part of a maximal nonnegative subspace $\mathcal{L}_{+}$invariant with respect to $A$.

Theorem 2.3. Let $E_{\lambda}$ be the e.s.f. of a $\pi$-self-adjoint operator A. Then there are a scalar Lebesgue measure $\mu_{\sigma}$, the Hilbert space $L_{\sigma}^{2}$ associated with $\mu_{\sigma}$, and a collection of $\mu_{\sigma}$-measurable scalar functions $\left\{\tilde{g}_{j}(t)\right\}_{j=1}^{k}$ such that if $\Delta=[a, b], 0 \notin \Delta$, the operator $\left.E(\Delta)\right|_{\widetilde{d}}$ is similar to the operator acting by the formulae

$$
\begin{gathered}
f(t) \longmapsto \chi_{\Delta}(t) f(t)+\sum_{j=1}^{k} \int_{\Delta} f(t) \overline{\widetilde{g}_{j}(t)} d \sigma(t) \cdot e_{j}, \\
e_{j} \longmapsto 0 \quad j=1,2, \ldots, k
\end{gathered}
$$

on the formal linear span of $L_{\sigma}^{2}$ and $\left\{e_{j}\right\}_{j=1}^{k}$, where $\left\{e_{j}\right\}_{j=1}^{k}$ is a basis on $\mathscr{\ell}_{1}, X_{\Delta}(t)$ is the indicator of $\Delta$, $x_{\Delta}(t) \tilde{g}_{j}(t) \in L_{\sigma}^{2}$ for $j=1,2, \ldots, n$, and

$$
\sum_{j=1}^{k} \alpha_{j} \tilde{g}_{j}(t) \in L_{\sigma}^{2} \Longrightarrow \alpha_{1}=\alpha_{2}=\cdots=\alpha_{k}=0 .
$$

Proof. According to Equality (2.5), the restriction $\left.E(\Delta)\right|_{\widetilde{d}}$ has the following matrix representation:

$$
\left.E(\Delta)\right|_{\widetilde{\mathscr{l}}}=\left(\begin{array}{cc}
0 & E_{12}(\Delta) \\
0 & E_{22}(\Delta)
\end{array}\right) .
$$

With no loss of generality (see Proposition 3.3 below for details), one can assume that $(\cdot, \cdot)=$ $[\cdot, \cdot]$ on $\mathscr{l}_{2}$ and $\mathscr{l}_{1} \perp \mathscr{l}_{2}$. Then the operator-valued function $F_{\lambda}:=E_{22}((-\infty, \lambda))$ represents an (orthogonal) resolution of the identity acting in the Hilbert space $\mathscr{L}_{2}$. From the classical theory, 
we know that there is a Hilbert space $L_{\sigma}^{2}$ of scalar functions that is a model space for $F_{\lambda}$ with some corresponding operator $W: L_{\sigma}^{2} \mapsto \mathscr{\ell}_{2}$ of similarity, that is,

$$
W^{-1} E_{22}(\Delta) W f(t)=\chi_{\Delta}(t) f(t) .
$$

Let $\left\{e_{j}\right\}_{1}^{n}$ be an orthonormalized basis in $\mathcal{L}_{1}$. Then, for every $\Delta=[a, b], 0 \notin \Delta$, and $j=1,2, \ldots n$, the expression $v_{j}^{\Delta} W f(t):=\left(E_{12}(\Delta) W f(t), e_{j}\right)$ defines a continuous linear functional in $L_{\sigma}^{2}$. Thus, for every $j=1,2, \ldots n$, there exists the $F$-measurable function $\tilde{g}_{j}(t)$ such that, for an arbitrary $\Delta=[a, b], 0 \notin \Delta$, and $f(t) \in L_{\sigma}^{2}$, the representation

$$
v_{j}^{\Delta} W f(t)=\int_{\Delta} f(t) \overline{\widetilde{g}(t)} d \sigma(t)
$$

holds. Since the spectral function $E$ is unbounded, $\int_{-1}^{1}|\tilde{g}(t)|^{2} d \sigma(t)=\infty$ for at least one $j=$ $1,2, \ldots, n$. It means that at least one function (maybe all) of the collection

$$
\left\{\widetilde{g}_{j}(t)\right\}_{j=1}^{n}
$$

is not in $L_{\sigma}^{2}$. Then we just need to prove (2.7).

Let $\sum_{j=1}^{n} \alpha_{j} \cdot \tilde{g}_{j}(t) \in L_{\sigma}^{2}$. Then the linear functional $\left(f \in L_{\sigma}^{2}\right)$

$$
\phi f:=\int_{\mathbb{R}} f(t) \overline{\sum_{j=1}^{n} \alpha_{j} \cdot \tilde{g}_{j}(t)} d \sigma(t)
$$

is bounded.

Next, by definition, the space $\widetilde{\mathscr{l}}$ is the closure of all vectors having the form

$$
W f(t)+\sum_{j=1}^{k} \int_{\mathbb{R}} f(t) \overline{\tilde{g}_{j}(t)} d \sigma(t) \cdot e_{j}
$$

with $f \in L_{\sigma}^{2}$ vanishing near zero; so, for every $x \in \mathscr{L}_{1}$, there is a sequence $\left\{f_{m}\right\}_{m=1}^{\infty}$ such that

$$
\lim _{m \rightarrow \infty}\left\|f_{m}\right\|_{L_{\sigma}^{2}}=0, \quad \lim _{m \rightarrow \infty} \sum_{j=1}^{k} \int_{\mathbb{R}} f_{m}(t) \overline{\widetilde{g}_{j}(t)} d \sigma(t) \cdot e_{j}=x
$$

The latter yields

$$
\left(x, \sum_{j=1}^{n} \alpha_{j} \cdot e_{j}\right)=\lim _{m \rightarrow \infty} \sum_{j=1}^{k} \int_{\mathbb{R}} f_{m}(t) \overline{\alpha_{j} \tilde{g}_{j}(t)} d \sigma(t)=\lim _{m \rightarrow \infty} \phi f_{m}=0 .
$$

Thus $\sum_{j=1}^{n} \alpha_{j} \cdot e_{j}=0$, therefore $\alpha_{1}=\alpha_{2}=\cdots=\alpha_{n}=0$.

Theorem 2.3 gives a possibility to construct a partial model representation for $A$ in a sense exposed below. With no loss of generality, one can assume that the Hilbert scalar product $(\cdot, \cdot)$ on $\mathscr{H}$ is such that the corresponding Gram operator re-denoted as $J$ (i.e., $[\cdot, \cdot]=(J \cdot, \cdot))$ has the properties $J=J^{-1}=J^{*}$. Let (see (2.5))

$$
\mathscr{t}_{0}=J \mathscr{d}_{1} .
$$


Then $\mathscr{l}_{0} \perp \widetilde{\mathscr{l}}$. Additionally, one can assume that the basis $\left\{e_{j}\right\}_{j=1}^{k}$ from Theorem 2.3 is orthonormalized, $[\cdot, \cdot]=(\cdot, \cdot)$ on $\mathscr{t}_{2}$ and $\mathscr{l}_{1} \perp \mathfrak{l}_{2}$. Let $P_{0}$ and $P_{2}$ be orthoprojections onto subspaces $\mathscr{L}_{0}$ and $\mathscr{d}_{2}$, respectively.

Theorem 2.4. Let a space $L_{\sigma}^{2}$ and functions $\left\{\widetilde{g}_{j}\right\}_{j=1}^{k}$ be related with the e.s.f $E_{\lambda}$ of a $\pi$-s.a. operator $A$ as in Theorem 2.3, and let $\tilde{L}_{\sigma}^{2}$ be a Hilbert space formed as the linear span of $L_{\sigma}^{2}$ and $\left\{\tilde{g}_{j}\right\}_{j=1}^{k}$, where the functions in $\left\{\tilde{g}_{j}\right\}_{j=1}^{k}$ are mutually orthogonal, have unit norm, and are orthogonal to the space $L_{\sigma}^{2}$ which conserves its Hilbert structure. Then $t \widetilde{g}_{j}$ belongs to the linear span of $\widetilde{L}_{\sigma}^{2}$ for every $j=1,2, \ldots, k$, and the compression $\left.\left(P_{0}+P_{2}\right) A\right|_{\mathscr{d}_{0} \oplus \mathscr{d}_{2}}$ of $A$ is similar to the multiplication operator by $t$ acting on $\widetilde{L}_{\sigma}^{2}$.

Theorem 2.4 is not new (see, e.g., a more general result in [9] and its applications in $[10,11])$ but it opens some ways to generalization and generates some open questions and problems. For instance, $(2.4)(\mathrm{c})$ is restrictive and must be dropped. This, in turn, implies a replacement of the space $\widetilde{L}_{\sigma}^{2}$ of scalar functions by a suitable space of vector-valued functions. Next, the passing from a single self-adjoint operator to a family of self-adjoint operators in the case of indefinite metric spaces has some difficulties which must be taken into account. Let us consider the following example.

Example 2.5. Let the union of systems $\left\{h_{1}, h_{2}, h_{3}\right\},\left\{g_{1}, g_{2}, g_{3}\right\}$, and $\left\{e_{k}\right\}_{k=1}^{\infty}$ give an orthonormalized basis in a Hilbert space $\mathcal{H}$. We define a fundamental symmetry $J$ on $\mathscr{d}$ by formulae

$$
J g_{j}=h_{j}, \quad J h_{j}=g_{j}, \quad j=1,2,3, \quad J e_{k}=e_{k}, \quad k=1,2, \ldots
$$

Thus $\mathscr{t}$ becomes a Pontryagin space. Next, we define operators $A$ and $B$ by formulae

$$
\begin{gathered}
A h_{1}=h_{2}, \quad A h_{2}=\sum_{k=1}^{\infty} \frac{1}{k} \cdot e_{k}, \quad A h_{3}=\sum_{k=1}^{\infty} \frac{1}{k^{4 / 5}} \cdot e_{k}, \quad A g_{1}=A g_{3}=0, \quad A g_{2}=g_{1}, \\
A e_{k}=\frac{1}{k} \cdot e_{k}+\frac{1}{k} \cdot g_{2}+\frac{1}{k^{4 / 5}} \cdot g_{3}, \quad k=1,2, \ldots, \\
B h_{1}=h_{3}, \quad B h_{2}=\sum_{k=1}^{\infty} \frac{1}{k^{4 / 5}} \cdot e_{k}, \quad B h_{3}=\sum_{k=1}^{\infty} \frac{1}{k^{3 / 5}} \cdot e_{k}, \quad B g_{1}=B g_{2}=0, \\
B g_{3}=g_{1}, \quad B e_{k}=\frac{1}{k^{4 / 5}} \cdot e_{k}+\frac{1}{k^{4 / 5}} \cdot g_{2}+\frac{1}{k^{3 / 5}} \cdot g_{3}, \quad k=1,2, \ldots
\end{gathered}
$$

It is easy to check that the operators $A$ and $B$ are $\pi$-self-adjoint and commute. Next, the eigen spectral function (e.s.f.) of $A$ has the form

$$
E_{\lambda}= \begin{cases}0, & \text { if } \lambda \leq 0 \\ I-\sum_{k:(1 / k) \geq \lambda}\left[\cdot, u_{k}\right] \cdot u_{k}, & \text { if } \lambda \in(0 ; 1] \\ I, & \text { if } \lambda>1\end{cases}
$$

where $u_{k}=e_{k}+k g_{1}+g_{2}+(k)^{1 / 5} e_{3}, k=1,2, \ldots$. Moreover, for every $\beta>\alpha>0$, we have the representations

$$
A E([\alpha, \beta))=\int_{\alpha}^{\beta} \lambda E(d \lambda), \quad B E([\alpha, \beta))=\int_{\alpha}^{\beta}(\lambda)^{4 / 5} E(d \lambda) .
$$


Therefore, $E_{\lambda}$ can be considered as an "e.s.f." of the family $\{A, B\}$; however,

$$
A \neq \int_{0}^{1+0} \lambda E(d \lambda), \quad B \neq \int_{0}^{1+0}(\lambda)^{4 / 5} E(d \lambda)
$$

because both integrals are nonconvergent. $\operatorname{Next}, \operatorname{dimKer}(A)=\operatorname{dimKer}(B)=2, \operatorname{but} \operatorname{Ker}(A) \neq$ $\operatorname{Ker}(B)$. Thus $B \notin \operatorname{Alg}(A)$ and $A \notin \operatorname{Alg}(B)$, where $\operatorname{Alg}(\cdot)$ means the weakly closed algebra generated by the identity operator and the corresponding $\pi$-self-adjoint operator. The same reasoning shows that there is no $\pi$-self-adjoint operator $C$ for which, simultaneously, $A \in$ $\operatorname{Alg}(C)$ and $B \in \operatorname{Alg}(C)$. Therefore, the description of the operator family in discussion cannot be reduced to a representation of one operator. At the same time the existence of the spectral function with (2.20) is not occasional (see Theorem 3.11 below) and gives a possibility to construct a partial model for the whole family.

So one of the problems is to generalize Theorem 2.4 to commutative families of selfadjoint operators in Pontryagin and Krein spaces. Next, it is clear that the space $\widetilde{L}_{\sigma}^{2}$ depends on the choice of (2.5), but the latter is not uniquely determined. Thus, another problem is a description of the ambiguity of $\widetilde{L}_{\sigma}^{2}$ or its analog with vector-valued functions. The present paper is devoted mainly to these two problems.

Of course, the direction marked by Theorem 2.4 is not unique in relation to the goal to construct a model representation of function type for operators in Pontryagin spaces. In particular, in [12], it was shown that a bounded cyclic self-adjoint operator $A$ in a Pontryagin space is unitary equivalent to the operator $A_{\Phi}$ of multiplication by the independent variable in some space $\Pi(\Phi)$ generated by a "distribution" $\Phi$. This distribution, in turn, is associated with a quasiintegral representation for the moment sequence $\left\{\left[A^{n} h, h\right]\right\}_{n=0}^{\infty}$, where $h$ is a cyclic vector. The model in consideration describes the behavior of $A$ on the whole Pontryagin space, and in this sense, it is complete. On the contrary, the model from Theorem 2.4 is a partial one; it describes only the part of $A$ that can be restored via its e.s.f.. It gives, indeed, an advantage to the model [12] but this advantage concerns mainly cyclic operators because in the case of a non-cyclic operator the corresponding space $\Pi(\Phi)$ contains matrix-valued functions, and this space has a more or less complicated structure. The latter, however, is not the main obstacle. The experience with a canonic representation of normal operators in finite-dimensional spaces with an indefinite metric (see, e.g., $[13,14]$ ) shows that any attempt to give an observable and, simultaneously, complete description for a commutative family of self-adjoint operators in spaces with indefinite metric with the rank of indefiniteness more than 2 has little chances to prosper. On the contrary, a partial model representation in the style of Theorem 2.4 can be generalized easily to commutative families of $\pi$-self-adjoint operators and to commutative families of $J$-self-adjoint operators of the so-called $D_{\kappa}^{+}$-class. A previous experience shows that, in spite of some incompleteness, this model was useful in some applications concerning a single $\pi$-self-adjoint operator, see Section 7 for more details.

\section{Preliminaries}

\subsection{Krein spaces}

Let $\mathscr{H}$ be a Krein space with an indefinite sesquilinear form $[\cdot, \cdot]$, let $\mathscr{H}=\mathscr{L}_{+}[\dot{+}] \mathscr{L}_{-}$be its canonical decomposition, let $P_{+}$and $P_{-}$be canonical projections $\mathscr{l}_{+}=P_{+} \mathscr{\ell}$ and $\mathscr{L}_{-}=P_{-} \mathscr{\ell}_{\ell}$, let 
$J=P_{+}-P_{-}$be a canonical symmetry, and let $(\cdot, \cdot)=[J \cdot, \cdot]$ be a canonical scalar product. Note that one of these canonical objects uniquely determines the others. Everywhere below, we fix, on $\mathscr{H}$, a unique form $[x, y]=(J x, y)$. At the same time, let us note that, in the question we consider, a concrete choice of Hilbert scalar product is not really essential. One needs only to fix the topology (defined by the above-mentioned scalar product) and the structure of $J$. Let us mention the following result (see [15]) concerning some redefinitions of a Hilbert structure in Krein spaces.

Proposition 3.1. Let $\mathscr{d}=\mathscr{L}_{+} \oplus \mathscr{H}_{-}$be a canonical decomposition of the Krein space $\mathscr{d}$ and let $\mathscr{d}=$ $\mathcal{L}_{+}[+] \mathcal{L}_{-}$be another canonical decomposition of the same space. Let the first decomposition define the canonical scalar product $(\cdot, \cdot)$ and let the second decomposition define the canonical scalar product $(\cdot, \cdot)_{1}$, that is, $[\cdot, \cdot]=(J \cdot, \cdot)=\left(J_{1} \cdot, \cdot\right)_{1}$. Then spectrum of the operator $Q=J_{1} J$ is strictly positive, and the operator $D: D^{2}=Q, \sigma(D) \subset(0,+\infty)$, has the properties

$$
\begin{aligned}
& \text { (a) }[D x, D y]=[x, y] \\
& \text { (b) }(D x, D y)_{1}=(x, y) \\
& \text { (c) } D \mathscr{L}_{+}=\mathcal{L}_{+}, \quad D \mathscr{L}_{-}=\mathcal{L}_{-} .
\end{aligned}
$$

Definition 3.2. The operator $D$ that we introduced in Proposition 3.1 is called the canonical isometry that maps the Krein space $\mathfrak{H}$ with the scalar product $(\cdot, \cdot)$ on the same Krein space $\mathfrak{H}$, but with the scalar product $(\cdot, \cdot)_{1}$. If it is necessary to exactly indicate the corresponding scalar products or the canonical decompositions of the space $\mathfrak{H}$, we replace $D$ with

$$
D_{\mathfrak{H}_{1},(\cdot,),(\cdot,)_{1}} \quad \text { or } \quad D_{\left\{\mathfrak{H}_{+}, \mathfrak{H}_{-}\right\}\left\{\mathfrak{L}_{+}, \mathfrak{L}-\right\}} \cdot
$$

Below nonnegative (especially maximal nonnegative) subspaces will play an important role. The set of all maximal nonnegative subspaces of the Krein space $\mathfrak{H}$ is denoted $\mathfrak{M}^{+}(\mathscr{\ell})$.

A subspace $\mathcal{L}$ is called pseudoregular [16] if it can be presented in the form

$$
\mathcal{L}=\widehat{L}+\mathcal{L}_{1}
$$

where $\widehat{\mathcal{L}}$ is a regular subspace and $\mathcal{L}_{1}$ is an isotropic part of $\mathcal{L}$ (i.e., $\mathcal{L}_{1}=\mathcal{L} \cap \mathcal{L}^{[\perp]}$ ).

Proposition 3.3. Let

(i) $\mathfrak{L}_{+} \in \mathfrak{M}^{+}(\mathfrak{H})$ and a pseudoregular subspace,

(ii) $\mathfrak{L}_{0}$ the isotropic subspace of $\mathfrak{L}_{+}$,

(iii) $(\cdot, \cdot)^{\prime}$ a scalar product on $\mathfrak{L}_{0}$ such that the norm $\sqrt{(x, x)^{\prime}}$ is equivalent to the original one,

(iv) $\mathfrak{L}_{-}=\mathfrak{L}_{+}^{[\perp]}$,

and

$$
\mathfrak{L}_{+}=\widehat{\mathfrak{L}}_{+}+\mathfrak{L}_{0}, \quad \mathfrak{L}_{-}=\widehat{\mathfrak{L}}_{-}+\mathfrak{L}_{0},
$$

where $\widehat{\mathfrak{L}}_{+}$and $\widehat{\mathfrak{L}}_{-}$are uniformly definite subspaces. Then one can define, on $\mathfrak{H}$, a canonical scalar product $(\cdot, \cdot)$ such that
(a) on $\mathfrak{L}_{0}: \quad(\cdot, \cdot) \equiv(\cdot, \cdot)^{\prime}$
(b) $\mathfrak{L}_{0} \perp \widehat{\mathfrak{L}}_{+}, \quad \mathfrak{L}_{0} \perp \widehat{\mathfrak{L}}_{-}$
(c) on $\widehat{\mathfrak{L}}_{+}: \quad(\cdot, \cdot)=[\cdot, \cdot]$
(d) on $\widehat{\mathfrak{L}}_{-}: \quad(\cdot, \cdot)=-[\cdot, \cdot]$. 
Definition 3.4. If a canonical scalar product of Krein space $\mathfrak{H}$ has (3.5), it is said to be compatible with (3.4) and the choice of the scalar product $(\cdot, \cdot)^{\prime}$ on $\mathfrak{L}_{1}$.

Definition 3.5. Let $\mathfrak{L}$ be a pseudoregular subspace and let

$$
\mathfrak{L}=\mathfrak{L}^{(1)}+\mathfrak{L}_{1}, \quad \mathfrak{L}=\mathfrak{L}^{(2)}+\mathfrak{L}_{1}
$$

be two different decompositions of $\mathfrak{L}$ as a direct sum of a regular subspace and the isotropic subspace. We will call the map $\mathcal{M}_{\mathfrak{L}, \mathfrak{L}^{(1)}, \mathfrak{L}^{(2)}}: \mathfrak{L}^{(1)} \mapsto \mathfrak{L}^{(2)}$, defined by the condition

$$
\left[\mathcal{M}_{\mathfrak{L}, \mathfrak{L}^{(1)}, \mathfrak{L}^{(2)}} x, y\right]=[x, y] \quad(\forall y \in \mathfrak{L}),
$$

the standard map associated with (3.6).

Remark 3.6. The map $\boldsymbol{M}_{\mathfrak{L}, \mathfrak{L}^{(1)}, \mathfrak{L}^{(2)}}$ is uniquely determined by the condition (3.7) since the subspaces $\mathfrak{L}^{(1)}$ and $\mathfrak{L}^{(2)}$ are projectionally complete and the subspace $\mathfrak{L}_{1}$ is neutral. This map preserves the sesquilinear form [., ·] (i.e., is $J$-isometric). Furthermore, the subspaces $\mathfrak{L}^{(1)}$ and $\mathfrak{L}^{(2)}$ can be presented in the form

$$
\mathfrak{L}^{(1)}=\mathfrak{L}_{+}^{(1)}[\dot{+}] \mathfrak{L}_{-}^{(1)}, \quad \mathfrak{L}^{(2)}=\mathfrak{L}_{+}^{(2)}[\dot{+}] \mathfrak{L}_{-}^{(2)},
$$

where $\mathfrak{L}_{+}^{(1)}$ is uniformly positive, $\mathfrak{L}_{-}^{(1)}$ is uniformly negative and

$$
\mathfrak{L}_{ \pm}^{(2)}=\mathcal{M}_{\mathfrak{L}, \mathfrak{L}^{(1)}, \mathfrak{L}^{(2)}} \mathfrak{L}_{ \pm}^{(1)}
$$

Then the union of (3.6) and (3.8) generates two decompositions of the type (3.4).

Define a special case of pseudoregular subspaces: a nonnegative (non-positive) subspace $\mathcal{L}$ is called a subspace of the class $h^{+}\left(h^{-}\right)$if it is pseudoregular and $\operatorname{dim} \mathcal{L}_{1}<\infty$ for $\mathcal{L}_{1}$ as in (3.3). In Pontryagin spaces, every subspace is pseudoregular, and every semidefinite subspace belongs to class $h^{+}$or $h^{-}$.

Here, the term "operator" means "bounded linear operator." By the symbol $B^{\#}$, we denote the operator $J$-adjoint $\left(J\right.$-a.) to an operator $B$. Thus, if $A=A^{\#}$, then $A$ is a $J$-s.a. operator. For an operator $A$, the symbol $\sigma(A)$ denotes its spectrum treated in the same way as in [17] or [8].

If an operator family $\mathfrak{Y}$ is such that the condition $A \in \mathfrak{Y}$ implies $A^{\#} \in \mathfrak{Y}$, then this family is said to be $J$-symmetric. An operator algebra $\mathfrak{A}$ is said to be a $W J^{*}$-algebra if it is closed in the weak operator topology, $J$-symmetric, and contains the identity $I$. The symbol Alg $\mathfrak{Y}$ means the minimal $W J^{*}$-algebra which contains $\mathfrak{Y}$.

One of the most important directions in the development of the operator theory is connected to the existence of invariant maximal semidefinite subspaces for certain operator sets (see [18] for references) and the study of the properties of the operators in such sets. A subspace $\mathcal{L}$ is said to be $A$-invariant (Y)-invariant) if it is invariant with respect to the operator $A$ (operator family $\mathfrak{Y}$ ). 
Proposition 3.7. Let $A$ be an operator that acts in $\mathfrak{H}$ and has an invariant pseudoregular subspace $\mathfrak{L}$, and let $\mathfrak{L}_{1}=\mathfrak{L} \cap \mathfrak{L}^{[\perp]}$ be also an A-invariant. Consider two different decompositions of the space $\mathfrak{L}$,

$$
\mathfrak{L}=\mathfrak{L}^{(1)}+\mathfrak{L}_{1}, \quad \mathfrak{L}=\mathfrak{L}^{(2)}+\mathfrak{L}_{1},
$$

as a direct sum of the isotropic part and a projectionally complete subspace. Let

$$
\left.A\right|_{\mathfrak{L}}=\left(\begin{array}{cc}
A^{(1)} & 0 \\
A_{1}^{(1)} & A_{1}
\end{array}\right),\left.\quad A\right|_{\mathfrak{L}}=\left(\begin{array}{cc}
A^{(2)} & 0 \\
A_{1}^{(2)} & A_{1}
\end{array}\right)
$$

be two matrix representations of $\left.A\right|_{\mathfrak{L}}$ corresponding to these decompositions. Then

$$
A^{(1)}=\left(\boldsymbol{M}_{\mathfrak{L}, \mathfrak{L}^{(1)}, \mathfrak{L}^{(2)}}\right) A^{(2)}\left(\boldsymbol{M}_{\left.\mathfrak{L}, \mathfrak{L}^{(1)}, \mathfrak{L}^{(2)}\right)^{-1},}\right.
$$

where $\boldsymbol{M}_{\mathfrak{L}, \mathfrak{L}^{(1)}, \mathfrak{L}^{(2)}}$ is the standard map associated with (3.10).

Proposition 3.8. Let

$$
\mathfrak{H}=\mathfrak{H}_{+}[+] \mathfrak{H}_{-}, \quad \mathfrak{H}=\mathfrak{L}_{+}[+] \mathfrak{L}_{-}
$$

be two different canonical decompositions of the Krein space $\mathfrak{H}$, and let $\mathfrak{H}_{+}$and $\mathfrak{L}_{+}$be invariant subspaces of a J-s.a. operator A. Then

$$
A \cdot D_{\left\{\mathfrak{H}_{+}, \mathfrak{H}_{-}\right\},\left\{\mathfrak{L}_{+}, \mathfrak{L}_{-}\right\}}=D_{\left\{\mathfrak{H}_{+}, \mathfrak{H}_{-}\right\},\left\{\mathfrak{L}_{+}, \mathfrak{L}_{-}\right\}} \cdot A,
$$

where $D_{\left\{\mathfrak{H}_{+}, \mathfrak{H}_{-}\right\},\left\{\mathcal{L}_{+}, \mathcal{L}_{-}\right\}}$are associated with Definition 3.2.

Definition 3.9. A $J$-symmetric operator family $\mathfrak{Y}$ belongs to the class $D_{\kappa}^{+}$if there is a subspace $\mathcal{L}_{+}$in $\mathscr{t}$ such that

(i) $\mathcal{L}_{+}$is $\mathfrak{Y}$-invariant,

(ii) $\mathcal{L}_{+} \in \mathfrak{M}^{+}(\mathscr{\ell}) \cap h^{+}$,

(iii) $\operatorname{dim}\left(\mathcal{L}_{+} \cap \mathcal{L}_{+}^{[\perp]}\right)=\kappa$.

Remark 3.10. If a $J$-symmetric family $\mathfrak{Y} \in D_{\kappa}^{+}$and $\mathcal{L}_{+}$is a $\mathfrak{Y}$-invariant subspace corresponding to Definition 3.9, then the pseudoregular subspace $\mathcal{L}_{+}^{[\perp]}$ is $\mathfrak{Y}$-invariant too.

\subsection{Spectral functions with peculiarities}

The spectral resolution for different operator classes is one of the important problems in the operator theory. Let us start with the following definition.

Let $\Lambda=\left\{\lambda_{k}\right\}_{1}^{n}$ be a finite set of real numbers and let $\Re_{\Lambda}$ be the family $\{X\}$ of all Borel subsets of $\mathbb{R}$ such that $\partial X \cap \Lambda=\varnothing$, where $\partial X$ is the boundary of $X$ in $\mathbb{R}$. Let $E: X \mapsto E(X)$ be a countably additive (in the weak topology) function, that maps $\Re_{\Lambda}$ to a commutative algebra of projections in a Hilbert space $\mathscr{t}$, where $E([\lambda-\epsilon, \lambda+\epsilon]) \neq 0$ for every $\lambda \in \Lambda$, and $\epsilon>0$, and, moreover, $E(\mathbb{R})=I$. $E(X)$ is called a spectral function on $\mathbb{R}$ with the peculiar spectral set $\Lambda$; the mention of $\Lambda$ can be omitted. The symbol Supp $(E)$ means the minimal closed subset of $\mathbb{R}$ such 
that $E(X)=0$ for every $X: X \subset \mathbb{R} \backslash S$ and $X \in \mathfrak{R}_{\Lambda}$. Besides the symbol $E$, we will use also, as a notation of a spectral function, the symbol $E_{\lambda}, \lambda \in \mathbb{R}$, where $E_{\lambda}=E((-\infty, \lambda))$.

A spectral function $E$, that acts in a Krein space, is said to be $J$-orthogonal or $J$-s.a. if $E(X)$ is a $J$-orthoprojection for every $X \in \mathfrak{R}_{\Lambda}$.

Let us recall the definition of scalar spectral operator with real spectrum [19]. An operator $A$ acting in a Hilbert space is said to be a scalar spectral operator if there exists a spectral function $E$ with empty peculiar spectral set $\Lambda$ such that, for every $X \in \mathfrak{R}_{\Lambda}: E(X) A=A E(X)$, $\sigma\left(\left.A\right|_{E(X)}\right) \subset \bar{X}$ and $A E(X)=\int_{X} \xi E(d \xi)$ in the weak sense.

Now, let $E$ be a spectral function with peculiar spectral set $\Lambda$. A scalar function $f(\xi)$ is said to be defined almost everywhere (with respect to $E$ ), to have a finite value almost everywhere, and so on, if the corresponding property holds almost everywhere in the weak sense on an arbitrary set $X \in \mathfrak{R}_{\Lambda}, X \cap \Lambda=\varnothing$. We will assume that the function $f(\xi)$ is not defined at $\Lambda$.

The following theorem was announced in [2] and proved in [20].

Theorem 3.11. Let $\mathfrak{Y} \in D_{\kappa}^{+}$be a commutative family of $J$-s.a. operators with real spectra. Then there exists a J-orthogonal spectral function $E_{\lambda}$ with a finite number of spectral peculiarities $\Lambda$ ( $\Lambda$ may be the empty set) such that the following conditions hold

(a) $E_{\lambda} \in A \lg \mathfrak{Y} \quad \forall \lambda \in \mathbb{R} \backslash \Lambda$;

(b) there is a nonnegative subspace $\mathcal{L}_{+}$, corresponding to Definition 3.9, for which the descomposition $E(\Delta) \mathscr{d}=E(\Delta) \mathcal{L}_{+}[+] E(\Delta) \mathcal{L}_{-}$holds, $\Delta$ being any closed segment $\Delta \subset \mathbb{R}$ satisfying $\Delta \in \mathfrak{R}_{\Lambda}$ and $\Delta \cap \Lambda=\varnothing$;

(c) for every operator $A \in \mathfrak{Y}$, there exists a defined almost everywhere and (uniformly) bounded function $\phi(\lambda)$ such that for every interval $\Delta \subset$ $\mathbb{R}, \Delta \in \mathfrak{R}_{\Lambda}, \Delta \cap \Lambda=\varnothing$, the decomposition $A E(\Delta)=\int_{\Delta} \phi(\lambda) E(d \lambda)$ is valid;

(d) the subspace $\widetilde{\mathscr{H}}=\underset{\Delta \in \mathfrak{R}_{\Lambda}, \Delta \cap \Lambda=\varnothing}{C \operatorname{Lin}}\{E(\Delta) \mathscr{d}\}$ is pseudoregular and its isotropic part has finite dimension;

(e) if $\lambda_{0} \in \Lambda$ and $\mathscr{H}_{\lambda_{0}}:=\bigcap_{\lambda_{0} \in \Delta} E(\Delta) \mathscr{H}$, then for every operator $A \in \mathfrak{Y}$ the set $\sigma\left(\left.A\right|_{\mathfrak{e}_{\lambda_{0}}}\right)$ is as ingletone $\left\{\mu_{A}\right\}$ and there is a positive integer $m_{A}$ such that $\left.\left(A-\mu_{A} I\right)^{m_{A}}\right|_{\mathscr{H}_{\lambda_{0}}}=0$;

(f) if $\lambda_{0} \in \Lambda$, then $\limsup _{\lambda \rightarrow \lambda_{0}}\left\|E_{\lambda}\right\|=\infty$ or at least for one $A \in \mathfrak{Y}$ the operator $\left.A\right|_{\mathscr{d}_{\Lambda_{0}}}$ is not a spectral operator of scalar type.

A spectral function $E$ with a peculiar spectral set $\Lambda$, satisfying (3.15), is called an eigen spectral function (e.s.f.) of the operator family $\mathfrak{Y}$.

Definition 3.12. Let $E_{\mathcal{\lambda}}$ be an e.s.f. of an operator family $\mathfrak{Y}$ and let an operator $A \in \mathfrak{Y}$ and a function $\phi(\lambda)$ be connected by the system of equalities from (3.15) (c). Then the function $\phi(\lambda)$ is said to be the portrait of the operator $A$, and the operator $A$ is said to be the original of $\phi(\lambda)$ in $\mathfrak{Y}$ (with respect to $E_{\curlywedge}$ ).

Let a spectral function $E$ with a peculiar spectral set $\Lambda$ be an e.s.f. of $\mathfrak{Y}$. If $\lambda \in \Lambda$, then $\lambda$ will be called a peculiarity of $\mathfrak{Y}$. Let $\lambda$ be a peculiarity. Fix a set $X \in \mathfrak{R}_{\Lambda}: X \cap \Lambda=\{\lambda\}$. The 
peculiarity $\lambda$ is called regular if the operator family $\{E(X \cap Y)\}_{Y \in \mathfrak{R}_{\Lambda}}$ is bounded; otherwise, it is called singular. Note that the notion of regular and singular peculiarities is correctly defined since the boundedness of the family $\{E(X \cap Y)\}_{Y \in \mathfrak{R}_{\Lambda}}$ does not depend on $X$.

\subsection{Some function spaces}

Assume that $\sigma(t)$ is a nondecreasing function defined on the segment $[-1 ; 1]$, continuous in the points $-1,0$, and 1, continuous (at least) from the left in all other points of the segment, and having an infinite number of growth points, where zero is one of these points. This function $\sigma(t)$ generates, on $[-1 ; 1]$, a Lebesgue-Stieltjes measure $\mu_{\sigma}$ and spaces $\left(L_{\sigma}^{2}, L_{\sigma}^{\infty}\right.$, etc.) of complex-valued functions. At the same time we will consider also some spaces of vectorvalued functions; so, from time to time, we will note, after a symbol of a space a symbol of a range for the functions forming this space, for instance, $L_{\sigma}^{2}(\mathbb{C})$. Next, let us consider a slightly different construction. Let $G(t)$ be a $\mu_{\sigma}$-measurable function defined a.e. on $[-1 ; 1]$ and such that a.e. $G(t) \geq 1$ for every $\tau \in(0 ; 1]$, it is true that $\int_{-1}^{-\tau} G(t) d \sigma(t)<\infty$ and $\int_{\tau}^{1} G(t) d \sigma(t)<\infty$, but $\int_{-1}^{1} G(t) d \sigma(t)=\infty$. Set

$$
v(\tau)= \begin{cases}\int_{-1}^{\tau} G(t) d \sigma(t), & \text { if } \tau \in[-1 ; 0] \\ -\int_{\tau}^{1} G(t) d \sigma(t), & \text { if } \tau \in[0 ; 1]\end{cases}
$$

The function $v(t)$ is nondecreasing in both segments $[-1 ; 0)$ and $(0 ; 1]$, but it is unbounded in neighborhoods of zero. Define, for it, a corresponding function space. Let $f(t)$ and $g(t)$ be arbitrary functions continuous in $[-1 ; 1]$ and vanishing in some neighborhoods (different in the general case for $f(t)$ and $g(t))$ of zero. Then the integral $\int_{-1}^{1} f(t) \overline{g(t)} d v(t)$ is well defined and generates a structure of pre-Hilbert space on the set of all such functions. The completion of the space will be denoted $L_{v}^{2}\left(\right.$ or $L_{v}^{2}(\mathbb{C})$ ). Note that, due to (3.16), the spaces $L_{\sigma}^{\infty}$ and $L_{v}^{2}$ form a Banach pair, so the space $L_{\sigma}^{\infty} \cap L_{v}^{2}$ is well defined (for details, see [21]).

Let us pass to some notation relating to direct integrals of Hilbert spaces and corresponding model descriptions of self-adjoint operators (see [22, Section 41]; [23, Chapter 7]; [24, Chapter 4.4]; [25, Chapter VII]). We will use definitions close to the "coordinate notation" given in [22]. A difference between [22] and the definitions that follow is related to the fact that direct integrals, here, will be used not only for a resolution of Hilbert spaces but also for a resolution of Krein spaces. Let $\mathcal{\varepsilon}$ be some separable Hilbert space ( $\mathcal{\varepsilon}$ can be finite dimensional), let $\left\{d_{j}\right\}_{1}^{\alpha}$ be an orthonormalized basis of this space, let $\sigma(t)$ be be the same as above. Let $\left\{\rho_{j}(t)\right\}_{1}^{\alpha}$ be a system of nonnegative $\mu_{\sigma}$-measurable functions defined almost everywhere (a.e) on the segment $[-1 ; 1]$ and such that every function of the system is the indicator of some set of nonzero measure and $\mu_{\sigma}\left\{t: \rho_{j}(t)=0, j=1,2, \ldots, \alpha\right\}=0$. Denote

$$
d \vec{\sigma}(t)=\sum_{j=1}^{\alpha} d_{j} \rho_{j}(t) d \sigma(t)
$$

In this case the sum in the right part of the formula is considered as a formal expression without any suggestion of its convergence or divergence. 
Here the space $M_{\vec{\sigma}}(\varepsilon)$ means the space of vector-valued functions $\{f(t)\}$ defined a.e. (with respect to $\mu_{\sigma}$ ) on the segment $[-1 ; 1]$ and taking values in $\varepsilon$ under the conditions

$$
f(t)=\sum_{j=1}^{\alpha} \beta_{j}(t) d_{j}
$$

where $\beta_{j}(t)$ runs the set of all $\mu_{\sigma}$-measurable a.e. finite scalar functions such that

$$
\begin{aligned}
& \text { (a) } \beta_{j}(t)=\rho_{j}(t) \beta_{j}(t), \quad j=1,2, \ldots, \alpha ; \\
& \text { (b) a.e. }\|f(t)\|_{\varepsilon}^{2}=\sum_{j=1}^{\alpha}\left|\beta_{j}(t)\right|^{2}<\infty .
\end{aligned}
$$

The topology on $M_{\vec{\sigma}}(\varepsilon)$ is introduced by a base for neighborhoods of zero, where any neighborhood of the base is defined by a couple of positive numbers $\epsilon$ and $\delta$ (the couples are different for the different neighborhoods) and contains all functions satisfying the condition $\mu_{\sigma}\left\{t:\|f(t)\|_{\mathcal{E}}^{2} \geq \delta\right\}<\epsilon$. Next, the symbol $L_{\vec{\sigma}}^{2}(\mathcal{E})$ means here a Hilbert space of functions $f(t) \in M_{\vec{\sigma}}(\varepsilon)$ such that $\int_{-1}^{1}\|f(t)\|_{\varepsilon}^{2} d \sigma(t)<\infty$.

The spaces $M_{\vec{\sigma}}(\mathcal{E})$ and $L_{\vec{\sigma}}^{2}(\mathcal{E})$ are said to be a standard space of measurable functions and a standard Hilbert space, respectively.

The choice of $\left\{d_{j}\right\}_{1}^{\alpha}$ in the construction of spaces $M_{\vec{\sigma}}(\mathcal{E})$ and $L_{\vec{\sigma}}^{2}(\mathcal{E})$ is not essential if

$$
\rho_{j}(t)=\rho_{l}(t) \quad \text { for } l, j=1,2, \ldots, \alpha .
$$

Spaces $L_{\vec{\sigma}}^{2}(\varepsilon)$ of this type correspond, in particular, to model representations of self-adjoint operators with uniform multiplicity (see [25]). So $M_{\vec{\sigma}}(\mathcal{E})$ and $L_{\vec{\sigma}}^{2}(\mathcal{E})$ are said to be spaces of uniform multiplicity $\alpha$ if conditions (3.20) are fulfilled.

If $L_{\vec{\sigma}}^{2}(\varepsilon)$ is not a space of uniform multiplicity, it can be represented as an orthogonal sum of spaces of uniform multiplicity. Note a special case of such representation.

Definition 3.13. A space $L_{\vec{\sigma}}^{2}(\mathcal{E})$ is said to be orderly decomposable on uniform components if (see (3.17))

$$
L_{\vec{\sigma}}^{2}(\mathcal{\varepsilon})=L_{\vec{\sigma}_{1}}^{2}\left(\varepsilon_{1}\right) \oplus L_{\vec{\sigma}_{2}}^{2}\left(\varepsilon_{2}\right) \oplus \ldots,
$$

where $\varepsilon=\oplus \sum_{j=1}^{\beta} \varepsilon_{j}$ (both $\beta=\infty$ and $\beta<\infty$ can occur), the above decomposition of $\varepsilon$ is concordant with the choice of the basis $\left\{d_{j}\right\}_{1}^{\alpha}$ in the sense that $\varepsilon_{j}=\operatorname{CLin}\left\{d_{j}\right\}_{d_{j} \in \varepsilon_{j}} L_{\vec{\sigma}_{j}}^{2}\left(\varepsilon_{j}\right)$ is a space of uniform multiplicity $\operatorname{dim} \varepsilon_{j}, \varepsilon_{j} \subset \varepsilon_{j+1}, \operatorname{dim} \varepsilon_{j}<\operatorname{dim} \varepsilon_{j+1}, \sigma_{j}(t)=\int_{-1}^{t} X_{j}(\tau) d \sigma(\tau), \chi_{j}(\tau)$ is the indicator of some $\mu_{\sigma}$-measurable set $X_{j}, \mu X_{j} \neq 0, d \vec{\sigma}_{j}(t)=\left(\sum_{l: d_{l} \in \varepsilon_{j}} d_{l}\right) d \vec{\sigma}_{j}(t), X_{j} \cap X_{l}=\varnothing$, $j, l=1,2, \ldots, \beta, j \neq l$.

Spaces orderly decomposable on uniform components play the key role in the theory of model representation for self-adjoint operators (for details, see, [25, Theorem VII.6]). Practically the same definition can be given for spaces $M_{\vec{\sigma}}(\mathcal{E})$.

Definition 3.14. $M_{\vec{\sigma}}(\mathcal{E})$ is said to be orderly decomposable on uniform components if

$$
M_{\vec{\sigma}}(\varepsilon)=M_{\vec{\sigma}_{1}}\left(\varepsilon_{1}\right)+M_{\vec{\sigma}_{2}}\left(\varepsilon_{2}\right)+\ldots,
$$

where $M_{\vec{\sigma}_{j}}\left(\mathcal{E}_{j}\right)$ is a space of uniform multiplicity, the rest of the elements in (3.22) are the same as in (3.21). 
We introduce some notation related to multiplication operators by scalar functions. Everywhere below we assume a scalar function $\varphi(t)$ to be defined a.e. on $[-1 ; 1], \mu_{\sigma^{-}}$ measurable, and a.e. bounded. For $f(t) \in M_{\vec{\sigma}}(\mathcal{E})$, set

$$
(\Phi f)(t)=\varphi(t) f(t) .
$$

It is clear that $(\Phi f)(t) \in M_{\vec{\sigma}}(\varepsilon)$, so (3.23) defines on $M_{\vec{\sigma}}(\varepsilon)$ the continuous operator $\Phi$ (= the multiplication operator by the function $\varphi(t))$. If $\varphi(t)$ satisfies some additional conditions, one can consider the operator $\Phi$ as acting simultaneously on different spaces. If, for instance, $\varphi(t)$ is continuous, then the operator $\Phi$ is well defined on every space $M_{\sigma}(\mathcal{E})$ independently of $\vec{\sigma}(t)$ and $\mathcal{\varepsilon}$. If $\varphi(t) \in L_{\sigma}^{\infty}(\mathbb{C})$, then $L_{\vec{\sigma}}^{2}(\mathcal{E})$ can also be taken as a domain of $\Phi$. So, if it is necessary, we will mention, simultaneously, the operator $\Phi$ and its domain using the notation $\{\Phi, \mathfrak{D}(\Phi)\}$, say, $\left\{\Phi, L_{\vec{\sigma}}^{2}(\varepsilon)\right\}$.

Let $X_{\tau}$ be the multiplication operator by the indicator $X_{[-1 ; \tau)}(t)$ of the set $[-1 ; \tau), X_{\tau}=$ $\left\{X_{\tau}, L_{\vec{\sigma}}^{2}(\varepsilon)\right\}$. Pass to the description of automorphisms acting on $L_{\vec{\sigma}}^{2}(\varepsilon)$ and commuting with operators $\mathrm{X}_{\tau}, \tau \in[-1 ; 1]$. Denote, by $\mathcal{G}_{t}$, the subspace of the space $\mathcal{\varepsilon}$ spanned by all vectors of the set $\left\{d_{j}\right\}_{j=1}^{\alpha}$ such that $\rho_{j}(t) \neq 0$ (see (3.17)). The following result is well known (e.g., Birman, Solomjak [23, Chapter 7]; [24, Theorem 4.4.6]; [22, Proposition 1, Subsection 2; Section 41]).

Proposition 3.15. Let $\mathcal{U}$ be a unitary operator commuting with $\left\{X_{\tau}, L_{\vec{\sigma}}^{2}(\varepsilon)\right\}$ for a.a. $\tau \in[-1 ; 1]$. Then there is an operator-valued weakly $\mu_{\sigma}$-measurable function $U_{t}$ defined a.e. on $[-1 ; 1]$ such that, for a.e. $t \in[-1 ; 1]$, the operator $U_{t}$ is unitary on $\mathcal{\varepsilon}$ and

$$
\begin{aligned}
& \text { (a) }(\mathcal{U} f)(t)=U_{t} f(t) ; \\
& \text { (b) } U_{t} \mathcal{G}_{t}=\mathcal{G}_{t} ; \\
& \text { (c) }\left.U_{t}\right|_{\mathcal{G}_{t}^{\perp}}=\left.I\right|_{\mathcal{G}_{t}^{\perp}} .
\end{aligned}
$$

Remark 3.16. The space $L_{\vec{\sigma}}^{2}(\mathcal{\varepsilon})$ is a complete linear set in $M_{\vec{\sigma}}(\mathcal{\varepsilon})$. So if initially an operator $U$ acts on $L_{\vec{\sigma}}^{2}(\mathcal{E})$ and satisfies conditions (3.24), its domain can be extended to $M_{\vec{\sigma}}(\mathcal{E})$ via the passage to the limit. Thus the operator $\left\{\mathfrak{U}, M_{\vec{\sigma}}(\mathcal{E})\right\}$ is a continuous bijective mapping.

\section{On a model representation for $J$-orthogonal spectral functions without peculiarities}

We define, on $L_{\vec{\sigma}}^{2}(\mathcal{E})$, an additional structure of a Krein space. Let $\partial$ be an operator acting on $L_{\vec{\sigma}}^{2}(\varepsilon)$, being at the same time self-adjoint, unitary and commuting with $X_{\tau}$ for a.e. $\tau \in[-1 ; 1]$. By Proposition 3.15, the operator 2 has the representation

$$
(2 f)(t)=J_{t} f(t), \quad t \in[-1 ; 1],
$$

where $J_{t}$ is self-adjoint a.e. on $[-1 ; 1]$. Let

$$
\left[f_{1}(t), f_{2}(t)\right]_{\varepsilon}=\left(J_{t} f_{1}(t), f_{2}(t)\right)_{\varepsilon} .
$$

Then the inner product

$$
\left[f_{1}(t), f_{2}(t)\right]_{L_{\vec{\sigma}}^{2}(\varepsilon)}=\int_{-1}^{1}\left[f_{1}(t), f_{2}(t)\right]_{\varepsilon} d \sigma(t) .
$$

converts the space $L_{\vec{\sigma}}^{2}(\mathcal{\varepsilon})$ to a Krein space. This Krein space is denoted $\partial-L_{\vec{\sigma}}^{2}(\varepsilon)$ and it is said to be a standard Krein space. 
Remark 4.1. The product $\left[f_{1}(t), f_{2}(t)\right]_{\varepsilon}$ is a.e. well defined both for $f_{1}(t), f_{2}(t) \in L_{\vec{\sigma}}^{2}(\varepsilon)$ and $f_{1}(t), f_{2}(t) \in M_{\vec{\sigma}}(\varepsilon)$.

A standard Krein space can be used for a model representation of a $J$-spectral function (=J-orth.sp.f.) $E_{\curlywedge}$ without peculiarities. For simplicity everywhere below, we will assume that

$$
E_{-1}=0, \quad E_{+1}=I, \quad E_{-1}=E_{-1+0} .
$$

Definition 4.2. Let $E_{\lambda}$ be a $J$-orth.sp.f. with the empty set of peculiarities. A space $\partial-L_{\vec{\sigma}}^{2}(\mathcal{E})$ is said to be a model space for $E_{\curlywedge}$ if, for some canonical scalar product on $\mathfrak{H}$, there is an isometric and $J$-isometric operator $W: \mathcal{2}-L_{\vec{\sigma}}^{2}(\mathcal{E}) \mapsto \mathfrak{H}$ such that, for every $\lambda \in[-1 ; 1]$,

$$
E_{\curlywedge}=W X_{\curlywedge} W^{-1},
$$

where, as above, $X_{\curlywedge}$ is the multiplication operator by the indicator $X_{[-1 ; \lambda)}(t)$ of the set $[-1 ; \lambda)$, $X_{\curlywedge}=\left\{X_{\curlywedge}, \partial-L_{\vec{\sigma}}^{2}(\varepsilon)\right\}$. The operator $W$ is said to be an operator of similarity.

Proposition 4.3. Every J-orth.sp.f. $E_{\curlywedge}$ with an empty set of peculiarities has a model space $\partial-L_{\vec{\sigma}}^{2}(\mathcal{E})$.

Proof. By [19, Lemma XV.6.2], one can define, on $\mathscr{t}$, a new scalar product (noncanonical in the general case) $(\cdot, \cdot)_{1}$, topologically equivalent to the initial scalar product $(\cdot, \cdot)$ and such that the spectral function $E_{\lambda}$, is orthogonal with respect to $(\cdot, \cdot)_{1}$. Then $[\cdot, \cdot]=(G \cdot, \cdot)_{1}$, where the operators $E_{\lambda}$ commute with $G$ for a.a. $\lambda \in[-1 ; 1]$. Now one can introduce, on $\mathscr{d}$, a new canonical scalar product $(\cdot, \cdot)_{2}=(|G| \cdot, \cdot)_{1}$, where $|G|=\left(G^{2}\right)^{1 / 2}$ is the module of $G$. The new scalar product generates the canonical decomposition $\mathscr{H}=\mathscr{H}_{+}^{(2)} \oplus_{2} \mathscr{\ell}_{-}^{(2)}$. It easy to show that the subspaces $\mathscr{H}_{+}^{(2)}$ and $\mathscr{H}_{-}^{(2)}$ are $E_{\lambda}$-invariant for a.a. $\lambda \in[-1 ; 1]$. Thus there are model spaces $2-L_{\vec{\sigma}_{+}}^{2}\left(\varepsilon_{+}\right)$and $2-L_{\vec{\sigma}_{-}}^{2}\left(\varepsilon_{-}\right)$for orth.sp.f. $\left.E_{\lambda}\right|_{\mathscr{L} \ell_{+}^{(2)}}$ and $\left.E_{\lambda}\right|_{\mathscr{\ell}_{-}^{(2)}}$, respectively. Without loss of generality one can assume that the scalar functions (see (3.17)) $\sigma_{+}(t)$ and $\sigma_{-}(t)$ are such that

$$
\begin{gathered}
\sigma_{+}(t)=\int_{-1}^{t} \rho_{+}(\lambda) d \sigma(\lambda), \quad \sigma_{-}(t)=\int_{-1}^{t} \rho_{-}(\lambda) d \sigma(\lambda), \quad \rho_{+}^{2}(\lambda)=\rho_{+}(\lambda), \\
\rho_{-}^{2}(\lambda)=\rho_{-}(\lambda), \quad \sigma(t)=\int_{-1}^{t}\left(\rho_{+}(\lambda)+\rho_{-}(\lambda)-\rho_{+}(\lambda) \rho_{-}(\lambda)\right) d \sigma(\lambda) .
\end{gathered}
$$

Put $\mathcal{\partial}-L_{\vec{\sigma}}^{2}(\varepsilon):=2-L_{\vec{\sigma}_{+}}^{2}\left(\varepsilon_{+}\right) \oplus \mathcal{\partial}-L_{\vec{\sigma}_{-}}^{2}\left(\mathcal{\varepsilon}_{-}\right)$. The rest is straightforward.

Remark 4.4. As is well known, a model representation for an orth.sp.f. is not uniquely determined. At the same time, all scalar measures for such representations in the case of the same orth.sp.f. are equivalent among themselves and the function (see (3.24)) $r(t)=$ $\operatorname{dim} \mathcal{G}_{t}$ does not depend on the choice of the model representation. This is the reason why a model representation for orth.sp.f., usually is taken in the class of Lebesgue spaces orderly decomposable on uniform components. In the case of $J$-orth.sp.f., the situation is slightly more complicated. Specifically, for all model representations for a fixed $J$-orth.sp.f., all admissible scalar measures are equivalent among themselves and two functions $r_{+}(t)=\operatorname{dimKer}\left(\left.\left(J_{t}-I\right)\right|_{\mathcal{C}_{t}}\right)$ and $r_{-}(t)=\operatorname{dimKer}\left(\left.\left(J_{t}+I\right)\right|_{\mathcal{C}_{t}}\right)$ do not depend among themselves and of the choice on the model representation. Conversely, if data include a class of equivalent measures, functions $r_{+}(t)$ and $r_{-}(t)$, then a $J$-orth.sp.f. with empty set of peculiarities can be restored up to 
$J$-isometric equivalence. In particular, one can choose a suitable function $\sigma(t)$, construct a space $2-L_{\vec{\sigma}_{+}}^{2}\left(\varepsilon_{+}\right)$using $\sigma(t)$ and $r_{+}(t)$, construct a space $2-L_{\vec{\sigma}_{-}}^{2}\left(\varepsilon_{-}\right)$using $\sigma(t)$ and $r_{-}(t)$, and put $\partial-L_{\vec{\sigma}}^{2}(\varepsilon):=\partial-L_{\vec{\sigma}_{+}}^{2}\left(\varepsilon_{+}\right) \oplus \partial-L_{\vec{\sigma}_{-}}^{2}\left(\mathcal{E}_{-}\right)$. Note that, even if the spaces $\partial-L_{\vec{\sigma}_{+}}^{2}\left(\varepsilon_{+}\right)$and $\partial-L_{\vec{\sigma}_{-}}^{2}\left(\varepsilon_{-}\right)$ are orderly decomposable on uniform components, the spaces $\partial-L_{\vec{\sigma}}^{2}(\varepsilon)$ in the general case do not have this property.

Proposition 4.5. Assume that $E_{\mathcal{\lambda}}$ is a J-orth.sp.f. with empty set of peculiarities, $\partial-L_{\vec{\sigma}}^{2}(\varepsilon)$ and $\partial^{\prime}-$ $L_{\vec{\sigma}}^{2}(\mathcal{\varepsilon})$ are two different standard Krein spaces such that each of them is a model space for $E_{\lambda}$ and is derived from the same space $L_{\vec{\sigma}}^{2}(\varepsilon)$ by canonical symmetries $\partial$ and $\partial^{\prime}$, respectively. Then, in $L_{\vec{\sigma}}^{2}(\varepsilon)$, there is a unitary operator $\mathfrak{U}$ satisfying (3.24) and such that $\partial^{\prime}=\mathfrak{U}^{-1} \partial \boldsymbol{U}$.

Proof. In the general case the operators of similarity $W$ and $W^{\prime}$ correspond to different canonical scalar products on the same space $\mathscr{H}$, say, $W: \mathcal{\partial}-L_{\vec{\sigma}}^{2}(\mathcal{\varepsilon}) \mapsto\{\mathscr{\ell},(\cdot, \cdot)\}$ and $W^{\prime}: \partial^{\prime}-L_{\vec{\sigma}}^{2}(\mathcal{\varepsilon}) \mapsto\left\{\mathcal{L},(\cdot, \cdot)_{1}\right\}$, where $(\cdot, \cdot):=[J \cdot, \cdot]$ and $(\cdot, \cdot)_{1}:=\left[J_{1} \cdot, \cdot\right]$. On the other hand thanks to Definition 4.2, $E_{\lambda}$ is self-adjoint simultaneously with respect to the scalar products $(\cdot, \cdot)$ and $(\cdot, \cdot)_{1}$, hence it commutes with $J$ and $J_{1}$. Thus, taking into account Proposition 3.1, we can assume that $(\cdot, \cdot)=(\cdot, \cdot)_{1}$. Then the required operator $\mathcal{U}$ can be defined by the formula $u:=W^{-1} W^{\prime}$. Indeed, first, this $\mathcal{U}$ is unitary because the canonical scalar product on $\mathscr{H}$ is the same for $W$ and $W^{\prime}$; second, for every function $f(t) \in L_{\vec{\sigma}}^{2}(\mathcal{E})$ is true,

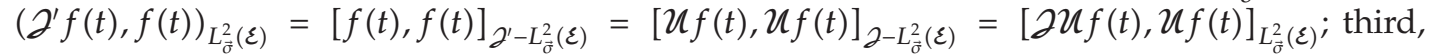
$\mathcal{u} \mathrm{X}_{\tau} f(t)=W^{-1} E_{\tau} W^{\prime} f(t)=\mathrm{X}_{\tau} \mathcal{U} f(t)$. The rest follows from Proposition 3.15.

The proof of the next proposition will be omitted because it contains the same wellknown ideas as the proof of Proposition 3.15.

Proposition 4.6. Let $\mathcal{M}$ be a J-unitary operator in a standard Krein space $2-L_{\vec{\sigma}}^{2}(\varepsilon)$ commuting with $\left\{X_{\tau}, \partial-L_{\vec{\sigma}}^{2}(\varepsilon)\right\}$ for a.a. $\tau \in[-1 ; 1]$. Then there is an operator-valued weakly measurable function $M_{t}$ defined a.e. on $[-1 ; 1]$ and taking values in the set of bounded operators acting in $\varepsilon$ such that, for a.a. $t \in[-1 ; 1]$,
(a) $\left(\mathcal{M f}_{f}(t)=M_{t} f(t)\right.$;
(b) $M_{t} \mathcal{G}_{t}=\mathcal{G}_{t}, M_{t} \mathcal{G}_{t}^{\perp}=\mathcal{G}_{t}^{\perp},\left.M_{t}\right|_{\mathcal{G}_{t}^{\perp}}=\left.I\right|_{\mathcal{G}_{t}^{\perp}}$;
(c) $\left[M_{t} x, M_{t} x\right]=[x, x] \quad \forall x \in \mathcal{E}$;
(d) $\operatorname{EssSup}_{t \in[-1 ; 1]}\left\|M_{t}\right\|_{\varepsilon}=\|\mathcal{M}\|_{L_{\vec{\sigma}}^{2}(\varepsilon)}$.

\section{Unbounded elements in Hilbert spaces}

First, let us consider the notion of unbounded elements.

Assume that $\mathscr{H}$ is a Hilbert space, $P_{t}$ is a resolution of the identity (= an orthogonal spectral function with the empty set of peculiar points) defined on the segment $[-1 ; 1]$, continuous in zero (with respect to the $w$-topology), and

(a) $P_{-1}=0, P_{1}=I$;

(b) for every $t \in[-1 ; 1]$ the unilateral limits $\mathrm{w}-\lim _{\mu \rightarrow t-0} P_{\mu}$ and $\underset{\mu \rightarrow t+0}{\mathrm{w}}-\lim _{\mu} P_{\mu}$ exist, where for definiteness $P_{t-0}=P_{t}$.

Set $P_{\lambda, \mu}=I+P_{\curlywedge}-P_{\mu+0}$, where $\lambda \in[-1 ; 0), \mu \in(0 ; 1]$. 
Next, let $x_{\lambda, \mu}$ be a mapping of the numerical set $[-1 ; 0) \times(0 ; 1]$ into $\mathscr{d}(\lambda \in[-1 ; 0), \mu \in$ $(0 ; 1])$. The function $x_{\lambda, \mu}$ is said to be conformed with $P_{t}$ if the following condition is fulfilled; for every $\lambda, \alpha \in[-1 ; 0), \mu, \beta \in(0 ; 1]$; the equality $P_{\lambda, \mu} x_{\alpha, \beta}=x_{\gamma, \delta}$ holds, where $\gamma=\min \{\lambda, \alpha\}$, $\delta=\max \{\mu, \beta\}$.

Note that $x_{\lambda, \mu}$ has the following property:

$$
\begin{aligned}
& \text { if } \sup _{\substack{\lambda \in[-1 ; 0) \\
\mu \in(0 ; 1]}}\left\{\left\|x_{\lambda, \mu}\right\|\right\}<\infty \text { then there is an element } x \in \mathscr{H} \text { such that for every } \\
& \lambda \in[-1 ; 0), \mu \in(0 ; 1] \text { the equality } x_{\lambda, \mu}=P_{\lambda, \mu} x \text { holds. }
\end{aligned}
$$

It is clear that the element $x$ from (5.2) is uniquely defined by $x_{\lambda, \mu}$ and can be found by the formula $x=\mathrm{w}-\lim _{\substack{\lambda \rightarrow-0 \\ \mu \rightarrow+0}} x_{\lambda, \mu}$.

Definition 5.1. A function $x_{\lambda, \mu}$, which is conformed with $P_{\lambda}$, is said to be an unbounded element conformed with $P_{\lambda}$ (or, if it cannot produce a misunderstanding or an unbounded element) if $\sup _{\lambda \in[-1 ; 0)}\left\{\left\|x_{\lambda, \mu}\right\|\right\}=\infty$.

$\mu \in(0 ; 1]$

Note that unbounded elements conformed with $P_{t}$ exist if and only if zero is a point of growth for $P_{t}$, that is, for every $\epsilon>0$ it is true that $P_{+\epsilon}-P_{-\epsilon} \neq 0$. Everywhere below in this section, this condition for $P_{t}$ is assumed to be fulfilled.

For brevity everywhere below unbounded elements will be denoted by symbols $\tilde{x}, \tilde{y}$, and so on. For $\lambda \in[-1 ; 0), \mu \in(0 ; 1]$, we set $x_{\lambda, \mu}:=P_{\lambda, \mu} \tilde{x}$.

Definition 5.2. Unbounded elements $\tilde{x}_{1}, \tilde{x}_{2}, \ldots, \tilde{x}_{k}$, conformed with a (common) resolution of the identity $P_{\lambda}$, are said to be linearly independent modulo $\mathscr{t}$ if every nontrivial linear combination of them is an unbounded element from $\mathcal{H}$.

Next, for every bounded $P_{t}$-measurable function $\phi(t)$, one can define, on $\mathscr{t}$, the following operator $\Phi$ :

$$
\Phi x:=\int_{-1}^{1} \phi(t) d P_{t} x
$$

Using the previous notation, rewrite the last formula as

$$
(\Phi \tilde{x})_{\lambda, \mu}=\int_{-1}^{\lambda} \phi(t) d P_{t} \tilde{x}+\int_{\mu}^{1} \phi(t) d P_{t} \tilde{x} .
$$

Representation (5.4) allows a possibility to treat the operator $\Phi$ in a more general sense; unbounded elements conformed with $P_{t}$ can be naturally included to the domain of $\Phi$. Note that the portrait of an unbounded element can be both a bounded element and an unbounded element. Moreover, $\phi(t)$ in (5.4) could also be taken unbounded. In this case the portrait of a bounded element, optionally, is an unbounded element.

Proposition 5.3. If a function $\phi(t)$ is unbounded on every subset of complete $E_{t}$-measure, then the vector space $\Phi$ de contains an infinite number of unbounded elements conformed with $P_{t}$ and a linearly independent modulo de. 
The last proposition shows (see also below, Proposition 5.4) that if we need to operate with a finite number of unbounded elements, then only bounded functions $\phi(t)$ are admissible in (5.4).

Until the end of the present section, the orthogonal resolution of the identity $P_{t}$ and a nondecreasing function $\sigma(t)$, such that $\mu_{\sigma}$-measurability on $[-1 ; 1]$ coincides with the $P_{t^{-}}$ measurability, will be fixed. Note that the existence of $\sigma(t)$ follows from the separability of $\mathscr{d}$ (see [26, Section 76, Theorem 1]).

We give an additional notation. Let $\left\{\tilde{x}_{j}\right\}_{1}^{k}$ be a fixed family of unbounded elements conformed with $P_{t}$ and a linearly independent modulo $\mathscr{H}$. Both the unbounded elements and the ordinary vectors from $\mathscr{H}$ can be considered as functions defined on $[-1 ; 0) \times(0 ; 1]$ and taking values in $\mathscr{t}$ (see (5.2)). The linear span of vectors from $\mathscr{t}$ and unbounded elements from $\left\{\tilde{x}_{j}\right\}_{1}^{k}$, consistently taken as functions on $[-1 ; 0) \times(; 1]$, is denoted $\widetilde{\mathscr{l}}$. Additionally $\widetilde{\mathscr{H}}$ will be considered as a Hilbert space, where $\mathscr{H}$ is a subspace with the the same scalar product that was given on $\mathscr{H}$ from the beginning, and unbounded elements from $\left\{\tilde{x}_{j}\right\}_{1}^{k}$ are mutually orthogonal and orthogonal to $\mathscr{H}$. The space $\widetilde{\mathscr{H}}$ is said to be an expansion of $\mathscr{H}$ (generated by $\left.\left\{\tilde{x}_{j}\right\}_{1}^{k}\right)$.

Next, using the system $\left\{\tilde{x}_{j}\right\}_{1}^{k}$, introduce the function $v(t)$;

$$
v(t)= \begin{cases}\sigma(t)+\sum_{j=1}^{k}\left\|P_{t} \tilde{x}_{j}\right\|^{2}, & \text { if } t \in[-1,0) \\ -\sigma(1)+\sigma(t)-\sum_{j=1}^{k}\left\|\left(P_{t}-I\right) \tilde{x}_{j}\right\|^{2}, & \text { if } t \in[0,1) .\end{cases}
$$

The connection between $P_{t}$ and $\sigma(t)$ implies that the function $v(t)$ introduced in (5.5) has (3.16). In this case the function $G(t)$ from (3.16) can be calculated directly through $v(t)$ and $\sigma(t)$.

Proposition 5.4. Let $\left\{\tilde{x}_{j}\right\}_{1}^{k}$ be a system of unbounded elements, forming together with $d$ the space $\widetilde{\mathscr{d}}$, let $\phi(t)$ be a $\mu_{\sigma}$-measurable function, let $\Phi$ be the operator defined by (5.4). Then $\Phi \widetilde{\mathscr{A}} \subset \mathscr{d}$ if and only if $\phi(t) \in L_{\sigma}^{\infty} \cap L_{v}^{2}$.

Proof. Sufficiency of the formulated condition is clear, so we will consider its necessity only. Note that, by virtue of Proposition 5.3, condition $\Phi \widetilde{\mathscr{H}} \subset \mathscr{H}$ implies the boundedness of the function $\phi(t)$. Next, since $\Phi \tilde{x}_{j} \in \mathscr{H}$ for $j=1,2, \ldots, k$, then, by (5.4) it is true that $\left\|\Phi \tilde{x}_{j}\right\|^{2}=$ $\int_{-1}^{1}|\phi(t)|^{2} d\left\|P_{t} \tilde{x}_{j}\right\|^{2}<\infty$ and, moreover, $\int_{-1}^{1}|\phi(t)|^{2} d \sigma(t)<\infty$. Thus (5.5) implies $\phi(t) \in L_{\sigma}^{\infty} \cap L_{v}^{2}$.

For future applications, both the cases $\Phi \widetilde{\mathscr{L}} \subset \mathscr{H}$ and

$$
\Phi \widetilde{\mathscr{A}} \subset \widetilde{\mathscr{U}}
$$

are important.

Proposition 5.5. Let $\left\{\tilde{x}_{j}\right\}_{1}^{k}$ be a system of unbounded elements, generating together with the space $\widetilde{d}$. Then there are no more than $k^{2} \mu_{\sigma}$-measurable functions $\phi(t)$ linearly independent modulo $L_{\sigma}^{\infty} \cap L_{v}^{2}$ such that (5.6) is fulfilled. 
Proof. Due to (5.6), every element from the system $\left\{\Phi \tilde{x}_{j}\right\}_{1}^{k}$ can be uniquely represented (modulo $\mathscr{\ell}$ ) as a linear combination of elements from $\left\{\tilde{x}_{j}\right\}_{1}^{k}$, that is,

$$
\Phi \tilde{x}_{j}=\sum_{m=1}^{k} c_{j m} \tilde{x}_{m}(\bmod \mathscr{\ell}), \quad j=1,2, \ldots, k .
$$

Thus the operator $\Phi$ generates the matrix

$$
C_{\Phi}=\left(c_{j m}\right)_{j, m=1}^{k}
$$

in addition, $\Phi \widetilde{\mathscr{A}} \subset \mathscr{H}$ if and only if $C_{\Phi}=0$. Hence functions comparable modulo $L_{\sigma}^{\infty} \cap L_{v}^{2}$ have the same matrix (5.8).

Remark 5.6. For the function $\phi(t) \equiv 1,(5.7)$ is always fulfilled. At the same time the example of the unbounded elements $\left\{(1)^{m}\right\}_{m=1}^{\infty}$ and $\left\{(i)^{m}\right\}_{m=1}^{\infty}$ from the space $\mathbf{l}_{2}$ shows that the case, where there are no functions different from a constant modulo $L_{\sigma}^{\infty} \cap L_{v}^{2}$ and simultaneously satisfying (5.6), is possible. Moreover, the estimation mentioned in Proposition 5.5 is excessive for every $k>1$ because two arbitrary matrices of the form (3.19) generated by (5.7) commute. For a discursion of the linear dimension of a group of commutative matrices, see, for instance, [27, Part 2, Section 10].

\section{A function model for a $J$-symmetric family of the class $D_{\kappa}^{+}$}

\subsection{Some remarks}

In this section a function model of $J$-symmetric family $\mathfrak{Y} \in D_{\mathcal{K}}^{+}$with real spectrum will be discussed. This model is defined with the help of an e.s.f. $E_{\lambda}$ of $\mathfrak{Y}$ (see Section 3). It is incomplete because the model describes the operator family restricted on some important subspaces (in particular, on the subspace $\widetilde{\mathscr{H}}($ see $(3.15)(\mathrm{d}))$ ) and not this family itself. By virtue of Theorem 3.11, it is clear that the general situation can be reduced to the case of $J$-orth.sp.f. $E_{\lambda}$ with a unique spectral peculiarity in zero. Furthermore, the case of a regular peculiarity is trivial because, under this condition, all operators from $\mathfrak{Y}$ are spectral in the sense of Dunford and have a finite-dimensional nilpotent part. Thus $E_{\lambda}$ is such that

$$
\begin{aligned}
& \text { (a) } E_{-1}=E_{-1+0}=0, \quad E_{+1}=I ; \\
& \text { (b) } \Lambda=\{0\} ; \\
& \text { (c) } \sup _{\lambda \in[-1 ; 1] \backslash\{0\}}\left\{\left\|E_{\lambda}\right\|\right\}=\infty .
\end{aligned}
$$

During the first stage, assume additionally that, for every closed interval $\Delta \subset[-1 ; 0) \cup(0 ; 1]$, the following condition holds:

$$
\text { if } E(\Delta) \neq 0 \text {, then the subspace } E(\Delta) \mathscr{l} \text { is positive. }
$$

Note that (6.2) does not necessarily mean that $\mathscr{H}$ is a Pontryagin space because the subspace $\widetilde{\mathscr{L}}^{[\perp]}$ can contain both positive and negative subspaces of infinite dimension. We introduce some notation. Let

$$
\mathscr{H}_{1}=\widetilde{\mathscr{H}} \cap \mathscr{\mathscr { H }}^{\perp]}, \quad \mathscr{H}_{2}=\mathscr{H}_{1}^{\perp} \cap \widetilde{\mathscr{H}}, \quad \mathscr{H}_{0}=J \mathscr{H}_{1},
$$

$P_{j}$ be an orthoprojection (in the sense of Hilbert spaces) onto $\mathscr{l}_{j}$,

$$
j=0,1,2, \quad \widetilde{E}_{\mathcal{\lambda}}:=\left.E_{\curlywedge}\right|_{\widetilde{\mathscr{l}}} .
$$


Note that, by virtue of $(6.1)(c)$, the equality $\mathscr{L}_{1} \neq\{0\}$ holds, and due to (6.2), the subspace $\mathscr{L}_{2}$ is uniformly positive (recall that the subspace $\mathscr{L}_{1}$ is finite dimensional). Without loss of generality one can assume that, on $\mathscr{\ell}_{2}$,

$$
(\cdot, \cdot)=[\cdot, \cdot] .
$$

Now, we pass to a detailed analysis of the structure of $E_{\lambda}$. First, let $X \in \mathfrak{R}_{\Lambda}$ and

$$
X \subset[-1 ; 1] \backslash\{0\} .
$$

Then

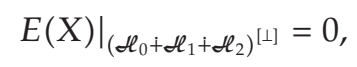

thus it is enough to study the behavior of $E(X)$ on the subspace

$$
\mathscr{L}_{0}+\mathscr{\ell}_{1}+\mathscr{\ell}_{2} \text {. }
$$

Assume that a canonical scalar product on $\mathscr{H}$ is such that $\left(\mathscr{\ell}_{0}+\mathscr{H}_{1}+\mathscr{H}_{2}\right)^{[\perp]} \perp\left(\mathscr{\ell}_{0}+\mathscr{H}_{1}+\mathscr{H}_{2}\right)$. In this case, (6.7) is an invariant subspace for $J$.

A direct verification shows that, if (6.5) holds, the operator $E(X)$ has the following matrix realization:

$$
\left.E(X)\right|_{\mathscr{H}_{0}+\mathscr{\ell}_{1}+\mathscr{\ell}_{2}}=\left(\begin{array}{ccc}
0 & 0 & 0 \\
E_{10}(X) & 0 & E_{12}(X) \\
E_{20}(X) & 0 & E_{22}(X)
\end{array}\right),
$$

with respect to (6.7), where

$$
\begin{gathered}
E_{10}(X)=E_{12}(X) E_{12}^{*}(X) V ; \quad E_{20}(X)=E_{12}^{*}(X) V ; \\
E_{22}^{2}(X)=E_{22}^{*}(X)=E_{22}(X) ; \quad E_{12}(X) E_{22}(X)=E_{12}(X),
\end{gathered}
$$

and the operator $V$ corresponds to the representation

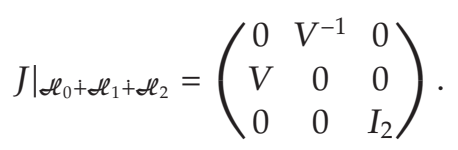

Let $X_{0}$ be a family of all sets under Condition (6.5). If $X, Y \in X_{0}$, then, for the operators $E(X)$ and $E(Y)$, the condition of the form (6.8) holds simultaneously. Since $E(X) E(Y)=E(Y) E(X)=$ $E(X \cap Y)$, then

$$
\begin{gathered}
E_{22}(X) E_{22}(Y)=E_{22}(Y) E_{22}(X)=E_{22}(X \cap Y) \\
E_{12}(X) E_{22}(Y)=E_{12}(Y) E_{22}(X)=E_{12}(X \cap Y) E_{22}(X \cap Y) .
\end{gathered}
$$

Now, we discuss the spectrum multiplicity of the family $\widetilde{E}_{\lambda}$. Recall that a subspace $\mathfrak{L}$ is said to be cyclic with respect to $\widetilde{E}_{\mathcal{\lambda}}$ if $\operatorname{CLin}_{\mathcal{L} \in[-1 ; 1] \backslash\{0\}}\left\{E_{\mathcal{\lambda}} \mathfrak{L}\right\}=\widetilde{\mathscr{L}}$. 
Definition 6.1. In what follows, a nonsingular multiplicity of $J$-orth.sp.f. $E_{\lambda}$ means the minimal dimension of all cyclic subspaces with respect to $\widetilde{E}_{\lambda}$.

Remark 6.2. Due to the choice of $\widetilde{\mathscr{H}}$ one can assume that a cyclic subspace $\mathfrak{L}$ is taken such that $\mathfrak{L} \subset \mathfrak{H}_{2}$, so the nonsingular multiplicity of $E_{\mathcal{\lambda}}$ coincides with the multiplicity (in the ordinary sense) of the (orthogonal) spectral function $\left.P_{2} E_{\lambda}\right|_{\mathscr{\ell}_{2}}$.

Proposition 6.3. If (6.1) and (6.2) are fulfilled and the nonsingular multiplicity of $E_{\lambda}$ is greater than $\operatorname{dim} \mathscr{H}_{1}$, then there is a decomposition $\mathscr{\mathscr { H }}=\mathscr{H}^{(1)}+\mathscr{H}^{(2)}$ such that $\mathscr{H}^{(1)}[\perp] \mathscr{H}^{(2)}, E_{\lambda} \mathscr{H}^{(1)} \subset \mathscr{H}^{(1)}$, $E_{\lambda} \mathscr{H}^{(2)} \subset \mathscr{H}^{(2)}$ for every $\lambda \in[-1 ; 1] \backslash\{0\}$, the subspace $\mathscr{H}^{(1)}$ is uniformly positive and the J-orth.sp.f. $\left.E_{\lambda}\right|_{\left(\mathscr{H}^{(1)}\right)^{[\perp]}}$ has nonsingular multiplicity less than or equal to $\operatorname{dim} \mathfrak{h}_{1}$.

Proof. Since $\mathscr{H}_{1} \subset \widetilde{\mathscr{H}}$ and (6.2) holds, there is a sequence of disjoint subsets $\left\{X_{k}\right\}_{1}^{\infty}$ such that a condition of the type (6.5) holds for all $k=1,2, \ldots, \cup_{k=1}^{\infty} X_{k}=[-1 ; 1] \backslash\{0\}$, and for every $k$, the equality $E_{12}\left(X_{k}\right) \mathscr{L}_{2}=\mathscr{l}_{1}$ is true. It is clear that $\operatorname{dim}\left(E_{12}^{*}\left(X_{k}\right) \mathscr{\ell}_{1}\right)=\operatorname{dim} \mathfrak{l}_{1}$. Let $\left\{u_{j}^{(k)}\right\}_{1}^{n}$ be some orthonormalized basis in $E_{12}^{*}\left(X_{k}\right) \mathscr{d}_{1}, k=1,2, \ldots$ Set

$$
u_{j}=\sum_{k=1}^{\infty}(1 / \sqrt{2})^{k} u_{j}^{(k)}, \quad j=1,2, \ldots, n ; \quad \mathscr{H}^{(3)}=\underset{Y \in \mathcal{X}_{0}}{\operatorname{CLin}}\left\{E_{22}(Y) u_{j}\right\}_{j=1}^{n}
$$

$\mathscr{H}^{(1)}=\left(\mathscr{H}^{(3)}\right)^{\perp} \cap \mathscr{H}_{2}, \mathscr{H}^{(2)}=\mathrm{CLin}_{Y \in \mathcal{X}_{0}}\left\{E(Y) \mathscr{H}^{(3)}\right\}$. We show that the subspaces $\mathscr{H}^{(1)}$ and $\mathscr{H}^{(2)}$ are as desired. First, we prove that, for every set $X \in \mathcal{X}_{0}$, the equality

$$
E_{12}(X) \mathscr{\ell}^{(1)}=\{0\} .
$$

holds. Indeed (see (6.11)), if $y \in \mathscr{H}^{(1)}$ and $z \in \mathscr{H}_{1}$, then

$$
\begin{aligned}
\left(E_{12}(X) y, z\right) & =\left(y, E_{12}^{*}(X) z\right) \\
& =\left(y, \sum_{k=1}^{\infty} E_{22}\left(X_{k}\right) E_{21}^{*}(X) z\right)=\sum_{k=1}^{\infty}\left(y, E_{22}\left(X \cap X_{k}\right) E_{21}^{*}\left(X_{k}\right) z\right) \\
& =\sum_{k=1}^{\infty}\left(y, E_{22}\left(X \cap X_{k}\right) \sum_{j=1}^{n} \alpha_{j}^{(k)} u_{j}^{(k)}\right)=0 .
\end{aligned}
$$

Next, it is clear that $\mathscr{H}^{(3)}$ is an invariant subspace with respect to the operators $E_{22}(X)$, therefore, the subspace $\mathscr{H}^{(1)}$ has the same property. Thus, taking into account (6.13), one can conclude that $\mathscr{\ell}^{(1)}$ is invariant with respect to $E_{\lambda}$.

Remark 6.4. Proposition 6.3 shows that, in some problems one can assume that the nonsingular multiplicity of $E_{\mathcal{\lambda}}$ is finite. At the same time, this hypothesis is not convenient in many cases because the decomposition $\widetilde{\mathscr{H}}=\mathscr{H}^{(1)}+\mathscr{H}^{(2)}$ is not uniquely defined and, moreover, the subspace $\mathscr{H}^{(1)}$ can be always extended saving all properties enumerated in Proposition 6.3. Basically, Proposition 6.3 gives a possibility to illustrate peculiarities of $J$-orth.sp.f. in question using $J$-orth.sp.f. with finite nonsingular multiplicity.

In addition to (6.3), set

$$
\widetilde{\mathscr{R}^{\uparrow}}=\mathscr{L}_{0} \oplus \mathscr{H}_{2}, \quad \widetilde{E}_{\lambda}=\left.E_{\lambda}\right|_{\widetilde{\mathscr{R}}^{\prime}} \quad \widetilde{E}_{\lambda}^{\uparrow}=\left.\left(P_{0}+P_{2}\right) E_{\lambda}\right|_{\widetilde{\mathbb{\ell}^{\top}}} .
$$


Now, let $L_{\vec{\sigma}}^{2}(\mathcal{E})$ and $M_{\vec{\sigma}}^{2}(\mathcal{E})$ be some standard Hilbert space and some standard space of measurable vector-functions, respectively, and let $\widetilde{g}_{1}(t), \widetilde{g}_{2}(t), \ldots, \widetilde{g}_{k}(t)$ be a finite collection of vector functions such that

(a) $\tilde{g}_{j}(t) \in M_{\vec{\sigma}}(\varepsilon), \quad j=1,2, \ldots, k$;

(b) the set $\left\{\tilde{g}_{j}(t)\right\}_{j=1}^{k}$ is a set of unbounded elements from $L_{\vec{\sigma}}^{2}(\mathcal{E})$ linearly independent modulo $L_{\vec{\sigma}}^{2}(\mathcal{\varepsilon})$ and conformed with $\mathrm{X}_{\tau}$.

Below $\widetilde{L}_{\vec{\sigma}}^{2}(\mathcal{\varepsilon}) \subset M_{\vec{\sigma}}(\mathcal{\varepsilon})$ is the linear span formed by $L_{\vec{\sigma}}^{2}(\varepsilon)$ and the collection $\left\{\widetilde{g}_{j}(t)\right\}_{j=1}^{k}$, where (see Section 5) the functions from $\left\{\tilde{g}_{j}(t)\right\}_{j=1}^{k}$ are assumed to be normalized, pairwise orthogonal, and orthogonal to $L_{\vec{\sigma}}^{2}(\varepsilon)$, that is, on $\widetilde{L}_{\vec{\sigma}}^{2}(\varepsilon)$, a structure of Hilbert space is defined. Note that, sometimes, $\widetilde{L}_{\vec{\sigma}}^{2}(\varepsilon)$ will be considered without its Hilbert structure as a vector subspace from $M_{\vec{\sigma}}(\varepsilon)$ but this case will be noted explicitly.

\subsection{Functional model of $E_{\lambda}$ (a special case)}

Theorem 6.5. If, for J-orth.sp.f. $E_{\lambda},(6.1)$ and (6.2) hold, and for a canonical scalar product $(\cdot, \cdot),(6.4)$ holds, then there exist a standard Hilbert space $L_{\vec{\sigma}}^{2}(\varepsilon)$, a collection $\left\{\tilde{g}_{j}(t)\right\}_{j=1}^{k}$ satisfying (6.16) which generates the expansion $\widetilde{L}_{\vec{\sigma}}^{2}(\mathcal{\varepsilon})$ of $L_{\vec{\sigma}}^{2}(\mathcal{\varepsilon})$, and an isometric operator $W: \widetilde{L}_{\vec{\sigma}}^{2}(\mathcal{\varepsilon}) \mapsto \widetilde{\mathscr{l}}, W L_{\vec{\sigma}}^{2}(\mathcal{E})=\mathscr{H}_{2}$ such that, for every $\lambda \in[-1 ; 1] \backslash\{0\}$, the following representations take place (see (6.10)):

$$
\widetilde{E}_{\lambda}=W \cdot X_{\lambda}^{*} \cdot(W)^{-1}, \quad W^{\uparrow}=\left(I_{2} \oplus V\right) W, \quad \tilde{E}_{\lambda}^{\uparrow}=W^{\uparrow} \cdot X_{\lambda} \cdot\left(W^{\uparrow}\right)^{-1},
$$

where $X_{\curlywedge}=\left\{X_{\curlywedge}, \tilde{L}_{\vec{\sigma}}^{2}(\mathcal{E})\right\}, k=\operatorname{dim} \mathscr{\ell}_{0}=\operatorname{dim} \mathfrak{\ell}_{1}$.

Proof. First, $\left.P_{2} E_{\lambda}\right|_{\mathscr{\ell}_{2}}$ is an orthogonal (in the ordinary Hilbert sense) spectral function, so there is a space $L_{\vec{\sigma}}^{2}(\mathcal{E})$ such that the operator $P_{2} E_{\lambda}$ is similar to the operator $X_{\lambda}=\left\{X_{\lambda}, L_{\vec{\sigma}}^{2}(\mathcal{E})\right\}$. Let a space $L_{\vec{\sigma}}^{2}(\mathcal{E})$ be already chosen and let an isometric operator $W_{2}: L_{\vec{\sigma}}^{2}(\mathcal{E}) \mapsto \mathscr{\ell}_{2}$ be such that $\left.P_{2} E_{\lambda}\right|_{\mathscr{\ell}_{2}}=W_{2} X_{\curlywedge} W_{2}^{-1}$. Now, it is necessary to find a collection of unbounded elements $\left\{\widetilde{g}_{j}(t)\right\}_{j=1^{\prime}}^{k}$ so that the corresponding space $\widetilde{L}_{\vec{\sigma}}^{2}(\mathcal{E})$ and the operator $W$ would be as desired. Choose, in $\mathscr{\ell}_{1}$, some orthonormalized basis $\left\{e_{j}\right\}_{j=1}^{k}$. Let $\Delta \subset[-1 ; 1] \backslash\{0\}$ be a closed interval. Consider the expression $\left(E(\Delta) x, e_{j}\right)$, where $x \in \mathfrak{l}_{2}$. It can be considered as a continuous linear functional acting in $\mathscr{\ell}_{2}$ or, equivalently, in $L_{\vec{\sigma}}^{2}(\mathcal{\varepsilon})$. By the well-known theorem of Riesz on the general representation of continuous linear functional, there is a function $g_{j, \Delta}(t) \in L_{\vec{\sigma}}^{2}(\mathcal{E})$ such that

$$
\left(E(\Delta) x, e_{j}\right)=\int_{-1}^{1}\left(f(t), g_{j, \Delta}(t)\right)_{\varepsilon} d \sigma(t)
$$

where $f(t)=W_{2}^{-1} x$ or (thanks to properties of the spectral function)

$$
\begin{gathered}
\left(E(\Delta) x, e_{j}\right)=\int_{\Delta}\left(f(t), g_{j, \Delta}(t)\right)_{\varepsilon} d \sigma(t), \\
g_{j, \Delta}(t)=0, \quad \text { if } t \notin \Delta .
\end{gathered}
$$


Condition (6.20) means that there is a function $\tilde{g}_{j}(t) \in M_{\vec{\sigma}}(\mathcal{\varepsilon})$ such that, for every interval $\Delta$ as defined above,

$$
g_{j, \Delta}(t)=\chi_{\Delta}(t) \tilde{g}_{j}(t),
$$

where $x_{\Delta}(t)$ is the indicator of the interval $\Delta$. The collection $\left\{\widetilde{g}_{j}(t)\right\}_{j=1}^{k}$ is as desired. We show this. First, we prove that the set $\left\{\tilde{g}_{j}(t)\right\}_{j=1}^{k}$ is linearly independent modulo $L_{\vec{\sigma}}^{2}(\varepsilon)$. Suppose the contrary, that is, suppose the existence of a collection of coefficients $\left\{\gamma_{j}\right\}_{j=1}^{k}$ such that

$$
0<\sum_{j=1}^{k}\left|r_{j}\right|, \quad \sum_{j=1}^{k} \gamma_{j} \tilde{g}_{j}(t) \in L_{\vec{\sigma}}^{2}(\varepsilon)
$$

Let $y=\sum_{j=1}^{k} \gamma_{j} e_{j}$. By the definition of the space $\widetilde{\mathscr{l}}$, there is a sequence $\left\{x_{m}\right\}_{1}^{\infty} \subset \operatorname{Lin}\{E(\Delta) \mathscr{H}\}$ such that $\left\|x_{m}-y\right\| \rightarrow 0$ for $m \rightarrow \infty$. Set $W_{2}^{-1}\left(P_{2} x_{m}\right)=f_{m}(t)$. Note that $f_{m}(t) \equiv 0$ in some neighborhood of zero, therefore, the representation

$$
\left\|x_{m}-y\right\|^{2}=\left(\left\|f_{m}(t)\right\|_{L_{\tilde{\sigma}}^{2}(\varepsilon)}\right)^{2}+\sum_{j=1}^{k}\left|\gamma_{j}-\int_{-1}^{1}\left(f_{m}(t), \tilde{g}_{j}(t)\right)_{\varepsilon} d \sigma(t)\right|^{2}
$$

is valid, hence $\lim _{m \rightarrow \infty}\left\|f_{m}(t)\right\|=0$,

$$
\lim _{m \rightarrow \infty} \int_{-1}^{1}\left(f_{m}(t), \widetilde{g}_{j}(t)\right)_{\varepsilon} d \sigma(t)=\gamma_{j}, \quad j=1,2, \ldots, k .
$$

These equalities and (6.22) imply

$$
\sum_{j=1}^{k}\left|\gamma_{j}\right|^{2}=\lim _{m \rightarrow \infty} \sum_{j=1}^{k} \bar{\gamma}_{j} \int_{-1}^{1}\left(f_{m}(t), \tilde{g}_{j}(t)\right)_{\varepsilon} d \sigma(t)=\lim _{m \rightarrow \infty} \int_{-1}^{1}\left(f_{m}(t), \sum_{j=1}^{k} \gamma_{j} \tilde{g}_{j}(t)\right)_{\varepsilon} d \sigma(t)=0,
$$

which is a contradiction, that is, (6.22) is impossible.

Second, form $\widetilde{L}_{\vec{\sigma}}^{2}(\mathcal{\varepsilon})$ according to the above procedure using the space $L_{\vec{\sigma}}^{2}(\mathcal{\varepsilon})$ and the collection of unbounded elements $\left\{\tilde{g}_{j}(t)\right\}_{j=1}^{k}$. Next, set

$$
\begin{gathered}
W f(t)=W_{2} f(t), \quad \text { if } f(t) \in L_{\vec{\sigma}}^{2}(\varepsilon) ; \\
W \tilde{g}_{j}(t)=e_{j}, \quad j=1,2, \ldots, k .
\end{gathered}
$$

In this case the dual operator $W^{\uparrow}$ is defined by the equalities

$$
\begin{gathered}
W^{\uparrow} f(t)=W_{2} f(t), \quad \text { if } f(t) \in L_{\vec{\sigma}}^{2}(\varepsilon) ; \\
W^{\uparrow} \widetilde{g}_{j}(t)=h_{j}, \quad \text { where } h_{j}=V^{-1} e_{j}, j=1,2, \ldots, k .
\end{gathered}
$$

Now, pass to (6.17). Assume (as above) that for a closed interval $\Delta$ the condition $\Delta \subset[-1 ; 1] \backslash$ $\{0\}$ holds. Then, in concordance with (6.8) and (6.19), one has $E_{12}(\Delta) x=\sum_{j=1}^{k}\left(x, z_{j}^{(\Delta)}\right) e_{j}$, where $z_{j}^{(\Delta)}=W g_{j, \Delta}(t), j=1,2, \ldots, k$, so $E_{20}(\Delta) h_{j}=z_{j}^{(\Delta)}, j=1,2, \ldots, k$. The last equalities, (3.21), and (3.23) imply (see the notation introduced in (6.15)) $W^{-1} \widetilde{E}^{\uparrow}(\Delta) W \widetilde{g}_{j}(t)=\chi_{\Delta}(t) \widetilde{g}_{j}(t), j=$ $1,2, \ldots, k$. Due to the choice of $L_{\vec{\sigma}}^{2}(\mathcal{E})$, the rest follows from (6.9) and (6.27). 
Definition 6.6. If $J$-orth.sp.f. $E_{\lambda}$ satisfies (6.1) and (6.2), and the space $\widetilde{L}_{\vec{\sigma}}^{2}(\varepsilon)$ has (6.17), then $\widetilde{L}_{\vec{\sigma}}^{2}(\mathcal{E})$ is said to be a basic model space for $E_{\lambda}$ (compatible with (6.3) and (6.15)), and the operator $W$ is said to be an operator of similarity (generated by $\widetilde{L}_{\vec{\sigma}}^{2}(\mathcal{\varepsilon})$ ).

Theorem 6.7. Let a space $\widetilde{L}_{\vec{\sigma}}^{2}(\mathcal{\varepsilon})$ be the expansion of a standard Hilbert space $L_{\vec{\sigma}}^{2}(\mathcal{\varepsilon})$ generated by a collection $\left\{\widetilde{g}_{j}(t)\right\}_{j=1}^{k}$ satisfying (6.16). Then there exist a Pontryagin space $\mathcal{L}$ and a J-orth.sp.f. $E_{\lambda}$ on He such that $\tilde{L}_{\vec{\sigma}}^{2}(\varepsilon)$ is a basic model space for $E_{\lambda}$.

Proof. Let $\mathscr{H}$ be the set of triples $(u, v, f(t))$ with $u, v \in \mathbb{C}^{k}$ and $f(t) \in L_{\vec{\sigma}}^{2}(\mathcal{\varepsilon})$. For $x_{1}=\left(u_{1}, v_{1}\right.$, $\left.f_{1}(t)\right)$ and $x_{2}=\left(u_{2}, v_{2}, f_{2}(t)\right)$, put

$$
\begin{aligned}
& \left(x_{1}, x_{2}\right)=\left(u_{1}, u_{2}\right)_{\mathbb{C}^{k}}+\left(v_{1}, v_{2}\right)_{\mathbb{C}^{k}}+\left(f_{1}(t), f_{2}(t)\right)_{L_{\bar{\sigma}}^{2}(\varepsilon)^{\prime}} \\
& {\left[x_{1}, x_{2}\right]=\left(u_{1}, v_{2}\right)_{\mathbb{C}^{k}}+\left(v_{1}, u_{2}\right)_{\mathbb{C}^{k}}+\left(f_{1}(t), f_{2}(t)\right)_{L_{\bar{\sigma}}^{2}(\varepsilon)} .}
\end{aligned}
$$

Next, let $\left\{e_{j}\right\}_{j=1}^{k}$ be the canonical basis in $\mathbb{C}^{k}$. Then, for intervals $\Delta=[\alpha, \beta)$, where $0 \notin \Delta$ and $\alpha \neq 0$, we put

$$
\begin{gathered}
E(\Delta)\left(e_{j}, 0,0\right)=\left(0, \sum_{m=1}^{k}\left(x_{\Delta}(t) \tilde{g}_{j}(t), \chi_{\Delta}(t) \tilde{g}_{m}(t)\right)_{L_{\tilde{\sigma}}^{2}(\varepsilon)} \cdot e_{m}, \chi_{\Delta}(t) \tilde{g}_{j}(t)\right) \\
\text { for } j=1,2, \ldots, k, \quad E(\Delta)(0, v, 0)=(0,0,0) \text { for every } v \in \mathbb{C}^{k} \\
E(\Delta)(0,0, f(t))=\left(0, \sum_{m=1}^{k}\left(\chi_{\Delta}(t) f(t), \chi_{\Delta}(t) \tilde{g}_{m}(t)\right)_{L_{\tilde{\sigma}}^{2}(\varepsilon)} \cdot e_{m}, \chi_{\Delta}(t) f(t)\right) .
\end{gathered}
$$

Direct verification shows that $E(\Delta)$ generates a $J$-orth.sp.f. with (6.1) and (6.2). Let us check that

$$
\mathscr{H}_{1}=\{(0, v, 0)\}_{v \in \mathbb{C}^{k}}, \quad \mathscr{H}_{2}=\{(0,0, f(t))\}_{f(t) \in L_{\bar{\sigma}}^{2}(\varepsilon)} .
$$

We denote, by the symbol $\left(L_{\vec{\sigma}}^{2}(\varepsilon)\right)_{0}$, the set of all functions from $L_{\vec{\sigma}}^{2}(\mathcal{\varepsilon})$ vanishing near zero, and for every $f(t) \in\left(L_{\vec{\sigma}}^{2}(\varepsilon)\right)_{0}$, we set $T_{1} f:=\sum_{m=1}^{k}\left(f(t), \widetilde{g}_{m}(t)\right)_{L_{\tilde{\sigma}}^{2}(\varepsilon)} \cdot e_{m}$. Then the space $\widetilde{\mathscr{l}}$ is the closure of the set

$$
\left\{\left(0, T_{1} f, f(t)\right)\right\}_{f(t) \in\left(L_{\tilde{\sigma}}^{2}(\varepsilon)\right)_{0}} .
$$

Let a system $\left\{v_{j}\right\}_{j=1}^{k}$ be an orthonormalized basis in $\mathbb{C}^{k}$. Then

$$
v_{j}=\sum_{m=1}^{k} \alpha_{m j} e_{m}
$$

and (6.31) can be rewritten as

$$
\left\{\left(0, \sum_{m=1}^{k}\left(f(t), \widehat{g}_{m}(t)\right)_{L_{\bar{\sigma}}^{2}(\varepsilon)} \cdot v_{m}, f(t)\right)\right\}_{f(t) \in\left(L_{\bar{\sigma}}^{2}(\varepsilon)\right)_{0}},
$$

where, by (6.32), $\widehat{g}_{m}(t)=\sum_{j=1}^{k} \bar{\alpha}_{j m} \widetilde{g}_{j}(t)$. Note that system $\left\{\widehat{g}_{m}(t)\right\}_{m=1}^{k}$ also has (6.16). 
Initially, the basis $\left\{v_{m}\right\}_{m=1}^{k}$ was arbitrary but now we pass to choose it in a special way. Since the elements from $\left\{\widetilde{g}_{m}(t)\right\}_{m=1}^{k}$ are unbounded, there is a sequence $\left\{f_{l}(t)\right\}_{l=1}^{\infty} \subset\left(L_{\vec{\sigma}}^{2}(\varepsilon)\right)_{0}$ such that $\lim _{l \rightarrow \infty}\left\|f_{l}(t)\right\|_{L_{\bar{\sigma}}^{2}(\varepsilon)}=0$ and $\left\|T_{1} f_{l}\right\|_{\mathbb{C}^{k}}=1$ for every $l$. Let $w_{l}=T_{1} f_{l}$. Since $w_{l} \in \mathbb{C}^{k}$, one can assume that there exists $\lim _{l \rightarrow \infty} w_{l}$, and put $v_{1}:=\lim _{l \rightarrow \infty} w_{l} \in \widetilde{\mathscr{A}}$. Now, we apply the same scheme for defining $v_{2}$. Let $T_{2} f:=\sum_{m=2}^{k}\left(f(t), \widehat{g}_{m}(t)\right)_{L_{\tilde{\sigma}}^{2}(\varepsilon)} \cdot v_{m}=T_{1} f-v_{1} \cdot\left(T_{1} f, v_{1}\right)_{\mathbb{C}^{k}}$. Then $\left(0, T_{2} f, f(t)\right) \in \widetilde{\mathscr{d}}$ for every $f(t) \in\left(L_{\vec{\sigma}}^{2}(\mathcal{\varepsilon})\right)_{0}$. Since the elements from $\left\{\hat{g}_{m}(t)\right\}_{m=2}^{k}$ are unbounded for every choice of $\left\{v_{j}\right\}_{j=1}^{k}$, there exists a (new) sequence $\left\{f_{l}(t)\right\}_{l=1}^{\infty} \subset\left(L_{\vec{\sigma}}^{2}(\varepsilon)\right)_{0}$ such that $\lim _{l \rightarrow \infty}\left\|f_{l}(t)\right\|_{L_{\bar{\sigma}}^{2}(\varepsilon)}=0$ and $\left\|T_{2} f_{l}\right\|_{\mathbb{C}^{k}}=1$ for every $l$. Let $w_{l}=T_{2} f_{l}$. Since $w_{l} \in \mathbb{C}^{k}$, one can assume that there exists $\lim _{l \rightarrow \infty} w_{l}$, and put $v_{2}:=\lim _{l \rightarrow \infty} w_{l} \in \widetilde{\mathscr{l}}$. Then $v_{1} \perp v_{2}$. The rest of the proof of (6.30) is now evident. Next, we put $W f(t)=(0,0, f(t))$ for every $f(t) \in \partial-L_{\vec{\sigma}}^{2}(\varepsilon)$, and $W \widetilde{g}_{j}(t)=\left(0, e_{j}, 0\right)$ for $j=1,2, \ldots, k$. The rest is straightforward.

We note that a basic model space for a given $J$-orth.sp.f. is not uniquely defined and discusses the arbitrariness for the choice of a concrete model. The construction of the basic model space was started from (6.3) and these depend on the choice of a canonical scalar product. So the first question is the following: does the basic model space depend on a choice of canonical scalar product? In order to answer this question, note, first, that $\mathfrak{d}_{1}$ does not depend on any canonical scalar product and is calculated using only the Hermitian sesquilinear form $[\cdot, \cdot]$ and $J$-orth.sp.f. $E_{\lambda}$. Second, one can consider the factor space $\widetilde{\mathscr{\ell}} / \mathscr{H}_{1}$. Then, by virtue of (6.2), the sesquilinear form $[\cdot, \cdot]$ generates in it the structure of Hilbert spaces, and the spectral function induced on $\widetilde{\mathscr{l}} / \mathscr{H}_{1}$ by the operator function $\widetilde{E}_{\mathcal{l}}$ is orthogonal. Third, by Definition 4.2 and Proposition 4.3, this induced function is similar to the spectral function $\left.P_{2} E_{\lambda}\right|_{\mathscr{\ell}_{2}}$. Summarizing all mentioned arguments, we obtain the following proposition.

Proposition 6.8. If $\widetilde{L}_{\vec{\sigma}}^{2}(\mathcal{E})$ is a basic model space for J-orth.sp.f. $E_{\lambda}$ satisfying (6.1) and (6.2), and $W$ is the corresponding operator of similarity, then the orth.sp.f. ${\stackrel{*}{E_{\lambda}}}$ induced on the Hilbert factor space ${ }^{*}:=$ $\widetilde{d} / \mathscr{L}_{1}$ by the J-orth.sp.f. $E_{\lambda}$, is similar to the operator family $X_{\lambda}$ acting in $L_{\vec{\sigma}}^{2}(\mathcal{E})$, and the corresponding operator of similarity $\stackrel{*}{W}: L_{\vec{\sigma}}^{2}(\mathcal{E}) \mapsto \stackrel{*}{\mathscr{\ell}}$ is induced by the operator $W$.

Thus the choice of $L_{\vec{\sigma}}^{2}(\varepsilon)$ as a source for constructing a basic model space $\widetilde{L}_{\vec{\sigma}}^{2}(\mathcal{\varepsilon})$ is realized in the same class of spaces as for the orth.sp.f. $\stackrel{*}{E}_{\mathcal{\lambda}}$ and does not depend on a canonical scalar product on $\widetilde{\mathscr{A}}$.

Proposition 6.9. Let spaces $\widetilde{L}_{\vec{\sigma}}^{2}(\varepsilon)$ and $\breve{L}_{\vec{\sigma}}^{2}(\mathcal{\varepsilon})$ be expansions of a space $L_{\vec{\sigma}}^{2}(\mathcal{\varepsilon})$ generating, respectively, by collections $\left\{\tilde{g}_{j}(t)\right\}_{j=1}^{k}$ and $\left\{\breve{g}_{j}(t)\right\}_{j=1}^{k}$ with $(6.16)$, let both $\widetilde{L}_{\vec{\sigma}}^{2}(\varepsilon)$ and $\breve{L}_{\vec{\sigma}}^{2}(\varepsilon)$ be a basic model space for the same J-orth.sp.f. $E_{\curlywedge}$ with (6.1) and (6.2), and let $W^{(1)}$ and $W^{(2)}$ be the corresponding operators of similarity. If for every function $f(t) \in L_{\vec{\sigma}}^{2}(\varepsilon)$, the relation

$$
\left(W^{(1)}-W^{(2)}\right) f(t) \in \mathscr{H}_{1},
$$

holds, then the spaces $\widetilde{L}_{\vec{\sigma}}^{2}(\mathcal{E})$ and $\breve{L}_{\vec{\sigma}}^{2}(\mathcal{E})$ coincide as vector subspaces in $M_{\vec{\sigma}}(\mathcal{E})$.

Proof. The complete proof will be divided in several stages. First, consider the case

$$
W^{(1)} L_{\vec{\sigma}}^{2}(\varepsilon)=W^{(2)} L_{\vec{\sigma}}^{2}(\varepsilon) .
$$


This equality means that, in particular, the basic model spaces $\widetilde{L}_{\vec{\sigma}}^{2}(\varepsilon)$ and $\breve{L}_{\vec{\sigma}}^{2}(\mathcal{\varepsilon})$ were constructed according to the procedure mentioned during the proof of Theorem 6.5 using the same decomposition $\widetilde{\mathscr{H}}=\mathscr{L}_{1}+\mathscr{\ell}_{2}$, but, generally speaking, a different choice of scalar products $(\cdot, \cdot)^{(1)},(\cdot, \cdot)^{(2)}$, and (or) basis in $\mathscr{H}_{1}$. Put

$$
e_{j}^{(1)}:=W^{(1)} \widetilde{g}_{j}(t), \quad e_{j}^{(2)}:=W^{(2)} \breve{g}_{j}(t), \quad j=1,2, \ldots, k
$$

Since the Hilbert structure of the spaces $\widetilde{L}_{\vec{\sigma}}^{2}(\mathcal{\varepsilon})$ and $\breve{L}_{\vec{\sigma}}^{2}(\mathcal{\varepsilon})$ is not in question, only the choice of the bases $\left\{e_{j}^{(1)}\right\}_{1}^{k}$ and $\left\{e_{j}^{(2)}\right\}_{1}^{k}$ in $\mathscr{L}_{1}$ is important (if scalar products are different then corresponding bases are necessarily different). Let

$$
\begin{gathered}
a_{j}^{(1)}(\Delta)=\left(E(\Delta) x, e_{j}^{(1)}\right)^{(1)}, \quad a_{j}^{(2)}(\Delta)=\left(E(\Delta) x, e_{j}^{(2)}\right)^{(2)}, \\
\Delta \subset[-1 ; 1] \backslash\{0\}, \quad x \in \mathscr{H}_{2}, \quad e_{j}^{(2)}=\sum_{l=1}^{k} \gamma_{j l} e_{l}^{(1)}, \quad j=1,2, \ldots, k .
\end{gathered}
$$

Then, by (6.19) and (6.21)

$$
a_{j}^{(1)}(\Delta)=\int_{\Delta}\left(f(t), \tilde{g}_{j}(t)\right)_{\varepsilon} d \sigma(t), a_{j}^{(2)}(\Delta)=\int_{\Delta}\left(f(t), \breve{g}_{j}(t)\right)_{\varepsilon} d \sigma(t),
$$

where $f(t)=\left(W^{(1)}\right)^{-1} x=\left(W^{(2)}\right)^{-1} x$. But $\left\{a_{j}^{(1)}(\Delta)\right\}_{1}^{k}$ and $\left\{a_{j}^{(2)}(\Delta)\right\}_{1}^{k}$ are the coefficients of decomposition for the same vector with respect to bases $\left\{e_{j}^{(1)}\right\}_{1}^{k}$ and $\left\{e_{j}^{(2)}\right\}_{1}^{k}$, respectively. Thus (6.37) gives the collection of equalities $a_{l}^{(2)}(\Delta)=\sum_{j=1}^{k} \gamma_{j l} a_{j}^{(1)}(\Delta), j=1,2, \ldots, k$, hence

$$
\int_{\Delta}\left(f(t), \breve{g}_{l}(t)\right)_{\varepsilon} d \sigma(t)=\int_{\Delta}\left(f(t), \sum_{j=1}^{k} \gamma_{j l} \widetilde{g}_{j}(t)\right)_{\varepsilon} d \sigma(t) .
$$

Since the segment $\Delta$ is arbitrary and $f(t)$ runs through the set of all functions from $L_{\vec{\sigma}}^{2}(\varepsilon)$, this equality gives

$$
\breve{g}_{l}(t)=\sum_{j=1}^{k} \gamma_{j l} \widetilde{\sigma}_{j}(t), \quad j=1,2, \ldots, k .
$$

Thus $\widetilde{L}_{\vec{\sigma}}^{2}(\varepsilon) \supset \breve{L}_{\vec{\sigma}}^{2}(\mathcal{\varepsilon})$. Since the spaces $\widetilde{L}_{\vec{\sigma}}^{2}(\varepsilon)$ and $\breve{L}_{\vec{\sigma}}^{2}(\mathcal{\varepsilon})$ have an equal status, the inverse inclusion is also true. So $\widetilde{L}_{\vec{\sigma}}^{2}(\varepsilon)$ and $\breve{L}_{\vec{\sigma}}^{2}(\varepsilon)$ coincide as subsets of $M_{\vec{\sigma}}(\varepsilon)$. The proposition is proved under (6.35).

For the second stage assume that the model spaces $\widetilde{L}_{\vec{\sigma}}^{2}(\mathcal{E})$ and $\breve{L}_{\vec{\sigma}}^{2}(\mathcal{E})$ are constructed using different decompositions of the space $\widetilde{\mathscr{l}}$, that is, $\widetilde{\mathscr{H}}=\mathscr{l}_{1}+\mathscr{l}_{2}^{(1)}, \widetilde{\mathscr{H}}=\mathscr{l}_{1}+\mathscr{l}_{2}^{(2)}$, but the basis in $\mathscr{\ell}_{1}$ and, moreover, the scalar product in $\mathscr{\ell}_{1}$ for both spaces are the same. Thus

$$
e_{j}=W^{(1)} \widetilde{g}_{j}(t)=W^{(2)} \breve{g}_{j}(t), \quad j=1,2, \ldots, k .
$$

Next, the space $\mathscr{H}_{2}^{(2)}$ can be represented in the form

$$
\mathscr{H}_{2}^{(2)}=\{x+Q x\}_{x \in \mathscr{H}_{2}^{(1)}}
$$


where $Q: \mathscr{H}_{2}^{(2)} \mapsto \mathscr{H}_{1}$ is a linear operator. It is clear that $Q x=\sum_{j=1}^{k} e_{j}\left(x, z_{j}\right)$, where $\left\{z_{j}\right\}_{1}^{k}$ is a vector set from $\mathscr{H}_{2}^{(1)}$. Note that the equality

$$
\left(W^{(1)}\right)^{-1} x=\left(W^{(2)}\right)^{-1}(x+Q x)
$$

holds for all $x \in \mathscr{H}_{2}^{(1)}$. Indeed, if $f(t):=\left(W^{(1)}\right)^{-1} x$, then by (6.42), $W^{(2)} f(t)=y+Q y$, where $y \in \mathscr{H}_{2}^{(1)}$. This implies $\left(W^{(1)}-W^{(2)}\right) f(t)=x-y-Q y$, and thanks to (6.34), one has $x=y$. Since $W^{(2)}$ is a one-to-one mapping, (6.43) is proved.

Now, let $x \in \mathscr{H}_{2}^{(1)} \cap\left(E(\Delta) \mathscr{H}+\mathscr{H}_{1}\right)$. Then by (6.15), the representation $\widetilde{\mathscr{H}}=\mathscr{H}_{1}+\mathscr{H}_{2}^{(1)}$ implies $E(\Delta) x=x+\sum_{j=1}^{k} e_{j}\left(x, s_{j}^{(1)}\right)$, where (cf., (6.19))

$$
s_{j}^{(1)}=W^{(1)} X_{\Delta}(t) \widetilde{g}_{j}(t), \quad j=1,2, \ldots, k,
$$

so if $\left(W^{(1)}\right)^{-1} x=f(t)$, then

$$
E(\Delta) x=x+\sum_{j=1}^{k} e_{j} \int_{\Delta}\left(f(t), \widetilde{g}_{j}(t)\right)_{\varepsilon} d \sigma(t) .
$$

The last equality can be rewritten as

$$
\begin{aligned}
E(\Delta) x & =(x+Q x)+\sum_{j=1}^{k} e_{j}\left(x, s_{j}^{(1)}-z_{j}\right) \\
& =(x+Q x)+\sum_{j=1}^{k} e_{j} \int_{\Delta}\left(f(t), \tilde{g}_{j}(t)-\zeta_{j}(t)\right)_{\varepsilon} d \sigma(t),
\end{aligned}
$$

where $\zeta_{j}(t)=\left(W^{(1)}\right)^{-1} z_{j} \in L_{\vec{\sigma}}^{2}(\varepsilon), j=1,2, \ldots, k$. On the other hand, by (6.15) and (6.43), the representation $\widetilde{\mathscr{l}}=\mathscr{H}_{1}+\mathscr{H}_{2}^{(2)}$ and the equality $E(\Delta) x=E(\Delta)(x+Q x)$ imply

$$
E(\Delta) x=(x+Q x)+\sum_{j=1}^{k} e_{j} \int_{\Delta}\left(f(t), \breve{g}_{l}(t)\right)_{\varepsilon} d \sigma(t) .
$$

The comparison between (6.46) and (6.47) gives

$$
\sum_{j=1}^{k} e_{j} \int_{\Delta}\left(f(t), \breve{g}_{l}(t)\right)_{\varepsilon} d \sigma(t)=\sum_{j=1}^{k} e_{j} \int_{\Delta}\left(f(t), \tilde{g}_{j}(t)-\zeta_{j}(t)\right)_{\varepsilon} d \sigma(t) .
$$

Since the function $f(t)$ is subjected only to the condition $f(t)=x_{\Delta}(t) f(t) \in L_{\vec{\sigma}}^{2}(\varepsilon)$ and the closed segment $\Delta$ is arbitrary, then $\breve{g}_{j}(t)=\tilde{g}_{j}(t)-\zeta_{j}(t), j=1,2, \ldots, k$. The proposition is proved under Hypothesis (6.41). The general case can be reduced to the two particular cases considered above, so the rest is straightforward.

The following proposition is a partial inverse of Proposition 6.9. 
Proposition 6.10. Let spaces $\widetilde{L}_{\vec{\sigma}}^{2}(\mathcal{E})$ and $\breve{L}_{\vec{\sigma}}^{2}(\mathcal{E})$ be the expansions of a space $L_{\vec{\sigma}}^{2}(\mathcal{E})$ generated, respectively, by collections $\left\{\tilde{g}_{j}(t)\right\}_{j=1}^{k}$ and $\left\{\breve{g}_{j}(t)\right\}_{j=1}^{k}$ satisfying (6.16). If $\widetilde{L}_{\vec{\sigma}}^{2}(\varepsilon)$ and $\breve{L}_{\vec{\sigma}}^{2}(\varepsilon)$ coincide as vector subspaces in $M_{\vec{\sigma}}(\varepsilon)$ and the space $\widetilde{L}_{\vec{\sigma}}^{2}(\mathcal{\varepsilon})$ is a basic model space for some J-orth.sp.f. $E_{\curlywedge}$ with (6.1) and (6.2), then the space $\breve{L}_{\vec{\sigma}}^{2}(\varepsilon)$ is also a basic model space for $E_{\lambda}$.

Proof. Let $W$ be the operator of similarity for $E_{\lambda}$ that corresponds to the basic model space $\widetilde{L}_{\vec{\sigma}}^{2}(\mathcal{E})$. The scheme of the proof will be the same as for Proposition 6.9; two particular cases will be considered and the superposition of these will give the general case.

So let a system $\left\{\breve{g}_{j}(t)\right\}_{j=1}^{k}$ be such that

$$
\tilde{g}_{j}(t)=\sum_{l=1}^{k} \gamma_{j l} \breve{g}_{j}(t), \quad j=1,2, \ldots, k
$$

Set $\mathscr{\ell}_{2}:=W L_{\vec{\sigma}}^{2}(\mathcal{E})$. The corresponding decomposition $\widetilde{\mathscr{H}}=\mathscr{\ell}_{1}+\mathscr{\ell}_{2}$ (generated by $W$ ) will be taken also for the construction of an operator of similarity $W^{\prime}$ corresponding to $\breve{L}_{\vec{\sigma}}^{2}(\varepsilon)$. First, set $W^{\prime} f(t):=W f(t)$ for $f(t) \in L_{\vec{\sigma}}^{2}(\varepsilon)$.

Next, for $x \in \mathscr{H}_{2}, x=W f(t)$, one has

$$
P_{1} E(\Delta) x=\sum_{j=1}^{k} e_{j} \cdot \int_{\Delta}\left(f(t), \tilde{g}_{j}(t)\right)_{\varepsilon} d \sigma(t)
$$

this, jointly with (6.49) implies

$$
P_{1} E(\Delta) x=\sum_{j=1}^{k} e_{j}^{\prime} \cdot \int_{\Delta}\left(f(t), \breve{g}_{j}(t)\right)_{\varepsilon} d \sigma(t),
$$

where $e_{j}^{\prime}=\sum_{l=1}^{k} \bar{r}_{l j} e_{l}, j=1,2, \ldots, k$. The vector system $\left\{e_{j}^{\prime}\right\}_{j=1}^{k}$ is taken as the new orthonormalized basis in $\mathscr{l}_{1}$ (so, generally speaking, the Hilbert structure on $\mathfrak{l}_{1}$ is redefined). Set $W^{\prime} \breve{g}_{j}(t):=e_{j}^{\prime}, j=1,2, \ldots, k$. Case (6.49) has been finished.

Now let $\tilde{g}_{j}(t)=\breve{g}_{j}(t)+\zeta_{j}(t), \zeta_{j}(t) \in L_{\vec{\sigma}}^{2}(\varepsilon), j=1,2, \ldots, k$. Put

$$
W^{\prime} f(t)=W f(t)+\sum_{j=1}^{k} e_{j} \cdot \int_{-1}^{1}\left(f(t), \zeta_{j}(t)\right)_{\varepsilon} d \sigma(t),
$$

where $f(t) \in L_{\vec{\sigma}}^{2}(\mathcal{\varepsilon})$ and $W^{\prime} \breve{g}_{j}(t)=e_{j}, j=1,2, \ldots, k$, that is, $\mathscr{\ell}_{2}^{\prime}=W^{\prime} L_{\vec{\sigma}}^{2}(\mathcal{\varepsilon})$. Note also that the Hilbert structure of $\mathscr{L}_{1}$ does not change. It is easy to check that the operator $W^{\prime}$ and the decomposition $\widetilde{\mathscr{H}}=\mathscr{H}_{1}+\mathscr{H}_{2}^{\prime}$ are as required.

Remark 6.11. The proof of Proposition 6.10 shows that, if $\widetilde{L}_{\vec{\sigma}}^{2}(\varepsilon)$ is a basic model space for $J$ orth.sp.f. $E_{\mathcal{\lambda}}$ with (6.1) and (6.2), $W$ is a corresponding operator of similarity, and $\widetilde{L}_{\vec{\sigma}}^{2}(\varepsilon)$ and $W$ are conformed with the decomposition $\widetilde{\mathscr{l}}=\mathscr{\ell}_{1}+\mathfrak{l}_{2}$, where $\mathscr{\ell}_{2}=W \widetilde{L}_{\vec{\sigma}}^{2}(\mathcal{\varepsilon})$, then for every different decomposition $\widetilde{\mathscr{A}}=\mathscr{L}_{1}+\mathscr{L}_{2}^{\prime}$ it is possible to find a new Hilbert structure of the vector set $\tilde{L}_{\vec{\sigma}}^{2}(\mathcal{E})$ (evidently this change does not touch $L_{\vec{\sigma}}^{2}(\mathcal{\varepsilon})$ ) and a new isometric map (an operator of similarity) $W^{\prime}: \widetilde{L}_{\vec{\sigma}}^{2}(\mathcal{\varepsilon}) \mapsto \widetilde{\mathscr{\ell}}$ such that $W^{\prime} L_{\vec{\sigma}}^{2}(\mathcal{\varepsilon})=\mathscr{H}_{2}^{\prime}$ and (e.g., $\left.(6.34)\right)\left(W-W^{\prime}\right) L_{\vec{\sigma}}^{2}(\mathcal{\varepsilon}) \subset \mathscr{H}_{1}$. An analogous proposition can be formulated for a change of the given Hilbert structure on $\mathfrak{l}_{1}$. 
Remark 6.12. Propositions 6.9 and 6.10 show that the Hilbert structure of the basic model space $\widetilde{L}_{\vec{\sigma}}^{2}(\varepsilon)$ introduced during an expansion of $L_{\vec{\sigma}}^{2}(\mathcal{\varepsilon})$ is not, in some sense, really important. On the other hand, an example mentioned below demonstrates that spaces $\widetilde{L}_{\vec{\sigma}}^{2}(\varepsilon)$ and $\breve{L}_{\vec{\sigma}}^{2}(\mathcal{\varepsilon})$ obtained as different expansions of a common space $L_{\vec{\sigma}}^{2}(\varepsilon)$ can be basic model spaces for the same $J$-orth.sp.f. $E_{\lambda}$ and be different vector subspaces in $M_{\vec{\sigma}}^{2}(\mathcal{\varepsilon})$. Thus (6.34) in Proposition 6.9 cannot be omitted.

Example 6.13. Let $\mathscr{H}$ be the totality of triples $(\gamma h, \xi e, f(t))$, where $h$ and $e$ are some fixed abstract vectors $\gamma, \xi \in \mathbb{C}, f(t) \in L_{t}^{2}(\mathbb{C})$, so the measure defining the space $L_{t}^{2}(\mathbb{C})$ is the standard Lebesgue measure. For $x_{1}=\left(\gamma_{1} h, \xi_{1} e, f_{1}(t)\right)$ and $x_{2}=\left(\gamma_{2} h, \xi_{2} e, f_{2}(t)\right)$, put

$$
\begin{aligned}
& \left(x_{1}, x_{2}\right)=\gamma_{1} \bar{\gamma}_{2}+\xi_{1} \bar{\xi}_{2}+\int_{-1}^{1} f_{1}(t) \bar{f}_{2}(t) d t \\
& {\left[x_{1}, x_{2}\right]=\gamma_{1} \bar{\xi}_{2}+\xi_{1} \bar{\gamma}_{2}+\int_{-1}^{1} f_{1}(t) \bar{f}_{2}(t) d t .}
\end{aligned}
$$

Next, let

$$
E_{\tau} x=\left(0,\left(\gamma \int_{-1}^{\tau}\left(1 / t^{2}\right) d t+\int_{-1}^{\tau}(1 / t) d t\right) e, X_{[-1 ; \tau)}(t)((\gamma / t)+f(t))\right),
$$

for $\tau \in[-1 ; 0)$, and let

$$
E_{\tau} x=\left(r h,\left(\xi-\int_{\tau}^{1} \frac{(\gamma+t f(t))}{t^{2}} d t\right) e, x_{[-1 ; \tau)}(t) f(t)+\frac{\gamma\left(X_{[-1 ; \tau)}(t)-1\right)}{t}\right),
$$

for $\tau \in(0 ; 1]$.

Direct verification shows that $E_{\tau}$ is a $J$-orth.sp.f. with (6.1), (6.2). In the capacity of a basic model space for $E_{\tau}$, one can take, first, the space $\widetilde{L}_{t}^{2}(\mathbb{C})$ generated by $L_{t}^{2}(\mathbb{C})$ and $\tilde{g}(t)=1 / t$, or, second, the space $\breve{L}_{t}^{2}(\mathbb{C})$ generated by $L_{t}^{2}(\mathbb{C})$ and $\breve{g}(t)=(1 / t) e^{i / t}$. In the first case, $W(\xi \tilde{g}(t)+f(t))=(0, \xi e, f(t))$ and in the second case, $W^{\prime}(\xi \breve{g}(t)+f(t))=\left(0, \xi e, e^{i / t} f(t)\right)(W$ and $W^{\prime}$ are the corresponding operators of similarity). At the same time, the spaces $\widetilde{L}_{t}^{2}(\mathbb{C})$ and $\breve{L}_{t}^{2}(\mathbb{C})$ form different vector subsets in $M_{t}(\mathbb{C})$ because $\left|\lim _{\alpha \rightarrow+0} \int_{\alpha}^{1} \tilde{g}(t) e^{-i / t} d t\right|<\infty$ and $\lim _{\alpha \rightarrow+0} \int_{\alpha}^{1} \breve{g}(t) e^{-i / t} d t=\infty$, that is, unbounded elements $\widetilde{g}(t)$ and $\breve{g}(t)$ are linearly independent modulo $L_{t}^{2}(\mathbb{C})$.

Theorem 6.14. Let spaces $\widetilde{L}_{\vec{\sigma}}^{2}(\varepsilon)$ and $\breve{L}_{\vec{\sigma}}^{2}(\varepsilon)$ be the expansions of a space $L_{\vec{\sigma}}^{2}(\varepsilon)$ generated, respectively, by collections $\left\{\widetilde{g}_{j}(t)\right\}_{j=1}^{k}$ and $\left\{\breve{g}_{j}(t)\right\}_{j=1}^{k}$ with (6.16). Then $\widetilde{L}_{\vec{\sigma}}^{2}(\varepsilon)$ and $\breve{L}_{\vec{\sigma}}^{2}(\varepsilon)$ are basic model spaces for a common J-orth.sp.f. $E_{\lambda}$ with (6.1) and (6.2) if and only if there is a unitary-valued function $U_{t}$ of the type (3.23) such that the sets $\widetilde{L}_{\vec{\sigma}}^{2}(\varepsilon)$ and $\left\{U_{t} \breve{f}(t)\right\}_{\breve{f}(t) \in L_{\vec{\sigma}}^{2}(\varepsilon)}$ coincide as vector spaces in $M_{\vec{\sigma}}(\varepsilon)$.

Proof

Necessity

By virtue of Remark 6.11, one can assume that, for model spaces $\widetilde{L}_{\vec{\sigma}}^{2}(\varepsilon), L_{\vec{\sigma}}^{2}(\varepsilon)$, and corresponding operators of similarity $W^{(1)}$ and $W^{(2)}$, there exist the same representation $\widetilde{\mathscr{d}}=$ $\mathscr{L}_{1} \oplus \mathscr{L}_{2}$ and the same Hilbert structure on $\mathscr{l}_{1}$. In this case, the operator $\mathcal{U}: \widetilde{L}_{\vec{\sigma}}^{2}(\mathcal{\varepsilon}) \mapsto \widetilde{L}_{\vec{\sigma}}^{2}(\mathcal{\varepsilon})$, $\mathcal{U}:=\left(W^{(2)}\right)^{-1} W^{(1)}$ is well defined. Direct verification gives $\mathcal{U}\left\{\mathrm{X}_{\tau}, \widetilde{L}_{\vec{\sigma}}^{2}(\mathcal{\varepsilon})\right\}^{*}=\left\{\mathrm{X}_{\tau}, \breve{L}_{\vec{\sigma}}^{2}(\mathcal{\varepsilon})\right\}^{*} \mathcal{U}$, 
which implies $\mathcal{U}\left\{\mathrm{X}_{\tau}, \widetilde{L}_{\vec{\sigma}}^{2}(\mathcal{\varepsilon})\right\}=\left\{\mathrm{X}_{\tau}, \breve{L}_{\vec{\sigma}}^{2}(\mathcal{\varepsilon})\right\} \mathcal{U}$. Since the subspace $L_{\vec{\sigma}}^{2}(\mathcal{E})$ is invariant both for $\mathrm{X}_{\tau}$ and $\mathcal{U}$, these two operators commute on $L_{\vec{\sigma}}^{2}(\varepsilon)$. Moreover, for every closed interval $\Delta \subset$ $[-1 ; 1] \backslash\{0\}$, the condition $X(\Delta) \tilde{g}_{j}(t) \in L_{\vec{\sigma}}^{2}(\mathcal{\varepsilon})$ holds for all $\tilde{g}_{j}(t), j=1,2, \ldots k$. The rest follows from Proposition 3.15.

\section{Sufficiency}

By Theorem 6.7, one can construct a Pontryagin space $\mathscr{H}$ and a $J$-orth.sp.f. $E_{\lambda}$ such that $\widetilde{L}_{\vec{\sigma}}^{2}(\varepsilon)$ is a basic model space for $E_{\lambda}$. The rest follows from (3.23) and Proposition 6.10.

The results obtained above show that the choice of a basic model space $\widetilde{L}_{\vec{\sigma}}^{2}(\varepsilon)$ for $J$ orth.sp.f. $E_{\curlywedge}$ with (6.1) and (6.2) is reduced (up to a finite number of functions) to the choice of a standard Hilbert space $L_{\vec{\sigma}}^{2}(\varepsilon)$. The last choice is not uniquely defined. The arbitrariness of the choice of $L_{\vec{\sigma}}^{2}(\mathcal{E})$ can be diminished if one takes in consideration only the standard Hilbert spaces orderly decomposable on uniform components. In the latter case, the choice is reduced (see [25, Theorem VII.6]) to the choice of scalar measure in the corresponding class of equivalent measures. Note that, if $L_{\vec{\sigma}}^{2}(\varepsilon)$ is orderly decomposable on uniform components, then it is naturally embedded to the space $M_{\vec{\sigma}}(\mathcal{E})$ and, therefore, this space is also orderly decomposable on uniform components. Moreover, the space $M_{\vec{\sigma}}(\mathcal{E})$ does not depend on $\sigma(t)$ but on the corresponding class of equivalent measures. This reasoning leads to the following theorem.

Theorem 6.15. Let $\widetilde{L}_{\vec{\sigma}}^{2}(\mathcal{\varepsilon})$ be a basic model space for J-orth.sp.f. $E_{\lambda}$ with (6.1) and (6.2), and let the space $L_{\vec{\sigma}}^{2}(\varepsilon)$ be orderly decomposable on uniform components. Then every basic model space for $E_{\lambda}$ constructed as an expansion of a standard Hilbert space orderly decomposable on uniform components has the following form;

$$
\left\{\frac{1}{\varphi(t)} U_{t} \tilde{f}(t)\right\}_{\tilde{f}(t) \tilde{L}_{\tilde{\sigma}}^{2}(\varepsilon)}
$$

where $\varphi(t)$ is a fixed scalar function satisfying the conditions $\varphi(t) \geq 0$ a.e. on $[-1 ; 1]$, and $\int_{-1}^{1} \varphi^{2}(t) d \sigma(t)<\infty$, and the operator-valued function $U_{t}$ is subject to (3.24). Conversely, every functional vector space of the form (6.56) is a basic model space for $E_{\lambda}$.

Remark 6.16. Let us consider an interpretation of Theorem 6.15 for the case of J-orth.sp.f. $E_{\lambda}$ with simple nonsingular multiplicity (in particular, $E_{\lambda}$ can be cyclic although this condition is not necessary). In this case, $L_{\vec{\sigma}}^{2}(\mathcal{E})$ converts to the space $L_{\sigma}^{2}(\mathbb{C})$ of scalar functions, and $U_{t}$ converts to the multiplication operator by a function $e^{i \vartheta(t)}$, where $\vartheta(t):[-1 ; 1] \mapsto[0 ; 2 \pi)$ is a $\mu_{\sigma}$-measurable function. Next, let $\left\{\tilde{g}_{j}(t)\right\}_{j=1}^{k}$ be a collection of unbounded elements generating the expansion $\widetilde{L}_{\sigma}^{2}(\mathbb{C})$ that is a basic model space for $E_{\lambda}$. Then every basic model space for $E_{\lambda}$ constructed as an expansion of a standard Hilbert space orderly decomposable on uniform components has, as a subset of $M_{\sigma}(\mathbb{C})$, the following form:

$$
\left\{\frac{1}{\varphi(t)}\left(f(t)+e^{i \vartheta(t)} \sum_{j=1}^{k} \gamma_{j} \widetilde{g}_{j}(t)\right)\right\}_{f(t) \in L_{\sigma}^{2}(\mathbb{C}), \gamma_{1}, \ldots, \gamma_{k} \in \mathbb{C}}
$$


Concluding this part, let us consider an estimation of the norm of a $J$-orthogonal projection $E(X)$, where the set $X$ satisfies (6.5) and the conventions given above on the choice of the canonical scalar product on $\mathscr{t}$ are preserved.

Proposition 6.17. If, under the mentioned above conditions, $E(X) \neq 0$, then $\|E(X)\|=1+\left\|E_{12}(X)\right\|^{2}$.

Proof. If $E_{12}(X)=0$, when the desired formula directly follows from (6.8), (6.9), so we assume that $E_{12}(X) \neq 0$. Let

$$
V^{-1} x \in \mathscr{H}_{0}, \quad y \in \mathscr{H}_{2}, \quad\|x\|^{2}+\|y\|^{2}=1
$$

Then $\|E(X)\|^{2}=\sup _{x, y}\left\{\left\|E(X)\left(V^{-1} x+y\right)\right\|^{2}\right\}$. On the other hand taking into account (6.9), (6.11), we have $\left\|E(X)\left(V^{-1} x+y\right)\right\|^{2}=\left\|E_{12}(X) E_{12}^{*}(X) x+E_{12}(X) y\right\|^{2}+\left\|E_{12}^{*}(X) x+E_{22}(X) y\right\|^{2} \leq(1+$ $\left.\left\|E_{12}(X)\right\|^{2}\right) \cdot\left\|E_{12}^{*}(X) x+E_{12}(X) y\right\|^{2}$. A first step is an estimation of $\left\|E_{12}^{*}(X) x+E_{22}(X) y\right\|^{2}$. Since $E_{22}(X)$ is an orthogonal projection, it is clear that it is enough to consider the case $E_{22}(X) y=y$. Next, $\left\|E_{12}^{*}(X) x+y\right\| \leq\left\|E_{12}^{*}(X)\right\|\|x\|+\|y\| \leq \sqrt{\left\|E^{*}(X)\right\|^{2}+1} \cdot \sqrt{\|x\|^{2}+\|y\|^{2}}=\sqrt{\left\|E^{*}(X)\right\|^{2}+1}$. Thus

$$
\left\|E(X)\left(V^{-1} x+y\right)\right\|^{2} \leq\left(1+\left\|E_{12}(X)\right\|^{2}\right)^{2} .
$$

The proof will be finished if vectors $x$ and $y$, satisfying (6.58) and converting (6.59) to an equality, can be found.

Since the operator $E_{12}^{*}(X)$ is finite dimensional, there is a vector $z \neq 0, z \in \mathscr{\ell}_{1}$ such that

$$
\left\|E_{12}^{*}(X) z\right\|=\left\|E_{12}^{*}(X)\right\|\|z\| .
$$

Let

$$
x=\frac{\left\|E_{12}(X)\right\| z}{\|z\| \sqrt{\left\|E^{*}(X)\right\|^{2}+1}}, \quad y=\frac{E_{12}^{*}(X) z}{\left\|E_{12}^{*}(X) z\right\| \sqrt{\left\|E^{*}(X)\right\|^{2}+1}} .
$$

Since $E_{22}(X) E_{12}^{*}(X)=E_{12}^{*}(X)($ see $(6.9)), y$ is well defined. Then

$$
\begin{aligned}
E_{12}^{*}(X) x+y & =\left(\left\|E_{12}(X)\right\|+\frac{1}{\left\|E_{12}^{*}(X)\right\|}\right) \cdot \frac{E_{12}(X) z}{\|z\| \sqrt{\left\|E^{*}(X)\right\|^{2}+1}} \\
& =\sqrt{\left\|E^{*}(X)\right\|^{2}+1} \cdot \frac{E_{12}(X) z}{\|z\| \cdot\left\|E_{12}^{*}(X)\right\|^{2}} .
\end{aligned}
$$

Finally (see (6.60)), $\left\|E_{12}(X) E_{12}^{*}(X) z\right\|=\left\|E_{12}(X)\right\|\left\|E_{12}^{*}(X) z\right\|$.

Proposition 6.18. Let $\widetilde{L}_{\vec{\sigma}}^{2}(\mathcal{E})$ be a basic model space for J-orth.sp.f. $E_{\curlywedge}$ with (6.1) and (6.2), and let $\left\{\tilde{g}_{j}(t)\right\}_{j=1}^{k}$ be the corresponding system with (6.16). Then

$$
\frac{1}{k} \int_{X} \sum_{j=1}^{k}\left\|\tilde{g}_{j}(t)\right\|_{\varepsilon}^{2} d \sigma(t) \leq\left\|E_{12}(X)\right\|^{2} \leq \int_{X} \sum_{j=1}^{k}\left\|\tilde{g}_{j}(t)\right\|_{\varepsilon}^{2} d \sigma(t) .
$$

Proof. Let $\left\{e_{j}\right\}_{j=1}^{k}$ be the orthonormalized basis in $\mathscr{H}_{1}$ corresponding to the system $\left\{\widetilde{g}_{j}(t)\right\}_{j=1}^{k}$. Then the operator $E_{12}^{*}(X) E_{12}(X)$ has, with respect to this basis the matrix representation $\left(\gamma_{m j}\right)_{k \times k}$ with the elements $\gamma_{m j}=\int_{X}\left(\tilde{g}_{m}(t), \tilde{g}_{j}(t)\right)_{\varepsilon} d \sigma(t)$. The rest is a usual estimation for the norm of a positive matrix. 


\subsection{A functional model for $E_{\curlywedge}$ (the general case)}

In the previous subsection, we studied $J$-orth.sp.f. $E_{\curlywedge}$ with (6.1), (6.2). Now, we turn to a more general case dropping (6.2). It implies that (6.9) and (6.11) must be substituted by some new ones. Let us conserve (6.3), (6.15). However, it is necessary to take into account that, now, generally speaking, the subspace $\mathscr{t}_{2}$ is indefinite. Recall that $J$-orth.sp.f. $E_{\lambda}$ belongs to the class $D_{\kappa}^{+}$, so there is an $E_{\lambda}$-invariant pair of $J$-orthogonal maximal semi-definite pseudoregular subspaces $\mathfrak{L}_{+}$and $\mathfrak{L}_{-}$with finite-dimensional isotropic part; moreover, due to (3.15)(b), we can assume that, for every closed interval $\Delta \subset[-1 ; 1] \backslash\{0\}$, the subspace $(E(\Delta) \mathscr{L}) \cap \mathfrak{L}_{+}$is positive and the subspace $(E(\Delta) \mathscr{L}) \cap \mathfrak{L}_{-}$is negative. Thanks to the hypothesis, the following subspaces are well defined:

$$
\widetilde{\mathscr{H}}_{+}=\underset{\Delta C[-1 ; 1] \backslash\{0\}}{\operatorname{Lin}}\left\{E(\Delta) \mathfrak{L}_{+}\right\}, \quad \widetilde{\mathscr{H}}_{-}=\underset{\Delta C[-1 ; 1] \backslash\{0\}}{\operatorname{CLin}}\left\{E(\Delta) \mathfrak{L}_{-}\right\} .
$$

Set

$$
\mathscr{H}_{2}^{+}=\mathscr{H}_{2} \cap \widetilde{\mathscr{H}}_{+}, \quad \mathscr{H}_{2}^{-}=\mathscr{H}_{2} \cap \widetilde{\mathscr{H}}_{-},
$$

and assume that a fundamental scalar product on $\mathscr{H}$ is simultaneously canonical for the subspace $\widetilde{\mathscr{H}} \oplus \mathscr{H}_{0}$ and, on the last space, compatible (see Definition 3.4) with the given decomposition of the subspaces

$$
\mathscr{H}_{1}+\mathscr{H}_{2}^{+}, \quad \mathscr{H}_{1}+\mathscr{H}_{2}^{-} \text {. }
$$

Thus

$$
\left.J\right|_{\mathscr{H}_{0}}+\mathscr{H}_{1}+\mathscr{H}_{2}=\left(\begin{array}{ccc}
0 & V^{-1} & 0 \\
V & 0 & 0 \\
0 & 0 & J_{2}
\end{array}\right),
$$

where the operator $V$ is the same as in (6.10), and $J_{2}$ is a canonical symmetry of the form $[\cdot, \cdot]$ on $\mathscr{t}_{2}$. Then, for $X$ satisfying (6.5), the elements of the matrix realization

$$
\left.E(X)\right|_{\mathscr{l}_{0}} \dot{+}_{\mathscr{H}_{1}} \dot{+}_{\mathscr{L}_{2}}=\left(\begin{array}{ccc}
0 & 0 & 0 \\
E_{10}(X) & 0 & E_{12}(X) \\
E_{20}(X) & 0 & E_{22}(X)
\end{array}\right)
$$

satisfy (cf. (6.9)) the following relations

$$
\begin{array}{cc}
E_{10}(X)=E_{12}(X) J_{2} E_{12}^{*}(X) V ; & E_{20}(X)=J_{2}^{*} E_{12}(X) V ; \\
E_{22}^{2}(X)=E_{22}^{*}(X)=E_{22}^{\#}(X)=E_{22}(X) ; & E_{12}(X) E_{22}(X)=E_{12}(X) .
\end{array}
$$

Here, $E_{22}^{\#}(X)$ is the operator $J$-adjoint to the operator $E_{22}(X)$ (note that now $\mathscr{L}_{2}$ is a Krein space). We note also that (6.11) remains valid.

Now, let $\partial-L_{\vec{\sigma}}^{2}(\varepsilon)$ be a standard Krein space (see Section 3 ) and let $\left\{\widetilde{g}_{j}(t)\right\}_{j=1}^{k}$ be a system of unbounded elements conformed with the operator-valued function $\mathrm{X}_{\tau}$ and linearly 
independent modulo $\partial-L_{\vec{\sigma}}^{2}(\varepsilon)$ (i.e., $\left\{\widetilde{g}_{j}(t)\right\}_{j=1}^{k}$ satisfies (6.16)). Denote, by $\partial-\widetilde{L}_{\vec{\sigma}}^{2}(\varepsilon)$, the linear span generated by the space $\partial-L_{\vec{\sigma}}^{2}(\varepsilon)$ and the system $\left\{\widetilde{g}_{j}(t)\right\}_{j=1}^{k}$. Define, on $\partial-\widetilde{L}_{\vec{\sigma}}^{2}(\varepsilon)$, structures of Hilbert and Krein spaces in the following way: on $\partial-L_{\vec{\sigma}}^{2}(\varepsilon)$ both structures coincide with the original structures, and functions of the system $\left\{\tilde{g}_{j}(t)\right\}_{j=1}^{k}$ are, by definition, positive (as elements of the Krein space), mutually orthogonal and $J$-orthogonal, normalized and $J$-normalized, and orthogonal and $J$-orthogonal to $\partial-L_{\vec{\sigma}}^{2}(\mathcal{\varepsilon})$. The space $\partial-\widetilde{L}_{\vec{\sigma}}^{2}(\mathcal{\varepsilon})$ is said to be the expansion of $\partial-L_{\vec{\sigma}}^{2}(\varepsilon)$ (generated by the collection $\left\{\tilde{g}_{j}(t)\right\}_{j=1}^{k}$ ).

Theorem 6.19. If a J-orth.sp.f. $E_{\lambda}$ satisfies (6.1) (but not (6.2)) and a scalar product on de is compatible with (6.66), then there are, first, a subspace $\partial-L_{\vec{\sigma}}^{2}(\mathcal{\varepsilon})$ and a system $\left\{\tilde{g}_{j}(t)\right\}_{j=1}^{k}$ with (6.16) forming together the space $\partial-\widetilde{L}_{\vec{\sigma}}^{2}(\varepsilon)$, and, second, an isometric J-isometric operator $W: \partial-\widetilde{L}_{\vec{\sigma}}^{2}(\varepsilon) \mapsto \widetilde{\mathscr{d}}$, $W L_{\vec{\sigma}}^{2}(\varepsilon)=\mathscr{\ell}_{2}$, such that, for every $\lambda \in[-1 ; 1]$,

$$
\tilde{E}_{\lambda}=W \cdot X_{\lambda}^{\#} \cdot(W)^{-1}, \quad W^{\uparrow}=\left(I_{2} \oplus V\right) W, \quad \tilde{E}_{\lambda}^{\uparrow}=W^{\uparrow} \cdot X_{\curlywedge} \cdot\left(W^{\uparrow}\right)^{-1},
$$

where $X_{\curlywedge}=\left\{X_{\curlywedge}, \partial-\widetilde{L}_{\vec{\sigma}}^{2}(\mathcal{E})\right\}, k=\operatorname{dim} \mathscr{\ell}_{0}=\operatorname{dim} \mathscr{\ell}_{1}$.

Proof. Since the scalar product $(\cdot, \cdot)$ on $\mathscr{L}_{2}$ is canonical and conformed with the decomposition $\mathscr{H}_{2}=\mathscr{L}_{2}^{+}[\dot{+}] \mathscr{H}_{2}^{-}$, the spectral function $P_{2} E_{\curlywedge}$ is simultaneously orthogonal and $J$-orthogonal. Then for $P_{2} E_{\lambda}$, by Proposition 4.3 and in concordance with Definition 4.2, there exist a model space $\partial-L_{\vec{\sigma}}^{2}(\varepsilon)$ and an operator of similarity $W_{2}$. The rest of the proof is analogous to the corresponding stage of the proof for Theorem 6.5 taking into account that (6.9) must be substituted by (6.69).

Remark 6.20. Under the conditions of Theorem 6.19, the operator function $\widetilde{E}_{\lambda}$ is similar not only to the operator function $\left\{X_{\lambda}^{\#}, \partial-\widetilde{L}_{\vec{\sigma}}^{2}(\varepsilon)\right\}$ but also to the operator-function $\left\{X_{\lambda}^{*}, \breve{L}_{\vec{\sigma}}^{2}(\varepsilon)\right\}$, where the space $\breve{L}_{\vec{\sigma}}^{2}(\varepsilon)$ is formed by the standard Hilbert space $L_{\vec{\sigma}}^{2}(\varepsilon)$ (it is the same as for $\partial-\widetilde{L}_{\vec{\sigma}}^{2}(\varepsilon)$ ) and the system of unbounded elements $\left\{\breve{g}_{j}(t)=\partial \widetilde{g}_{j}(t)\right\}_{j=1}^{k}$. Thus the properties of the operatorvalued function $\widetilde{E}_{\mathcal{\lambda}}$ (as well as for $\widetilde{E}_{\lambda}^{\uparrow}$ ) do not depend on either (6.2) is fulfilled or not for the operator-valued function $E_{\lambda}$. However this dependence exists for the $J$-orth.sp.f. $E_{\lambda}$, and this fact is connected with the different structure of the operator $E_{10}(X)$ in (6.9) and (6.69). Note, in particular, that, if (6.2) is not fulfilled, then the estimation for the norm of operator $E_{12}(X)$ given in Proposition 6.18 remains true, but at the same time, the equality for $\|E(X)\|$ from Proposition 6.17 is, generally speaking, incorrect.

Example 6.21. Take the space $\mathscr{H}$ coinciding with $\mathbf{1}_{2}$, denote $\left\{u_{m}\right\}_{m=1}^{\infty}$ the canonical basis of this space, that is, $u_{1}=(1,0,0, \ldots), u_{2}=(0,1,0, \ldots), \ldots$, and define the canonical symmetry $J$ by the equalities $J u_{0}=u_{1}, J u_{1}=u_{0}, J u_{2 m+1}=u_{2 m+1}, J u_{2 m}=-u_{2 m}, m=1,2, \ldots$, and the spectral function $E_{\curlywedge}$ by the relations

(i) $E_{\lambda}=0$ for every $\lambda<0$;

(ii) if $\lambda>0$, then $\left(I-E_{\curlywedge}\right) u_{0}=\sum_{1 / m \in[\lambda ; 1)}\left(u_{2(m-1)}-u_{2 m-1}\right),\left(I-E_{\curlywedge}\right) u_{1}=0,\left(I-E_{\curlywedge}\right) u_{2(m-1)}=$ $u_{2(m-1)}+u_{1},\left(I-E_{\lambda}\right) u_{2 m-1}=u_{2 m-1}+u_{1}$ for $1 / m \in[\lambda ; 1)$, and $\left(I-E_{\lambda}\right) u_{2(m-1)}=(I-$ $\left.E_{\curlywedge}\right) u_{2 m-1}=0$ for $1 / m \notin[\lambda ; 1)$. 
For $\Delta_{m}=[1 / m ; 1]$, one has $\left\|E_{12}\left(\Delta_{m}\right)\right\|=\sqrt{2(m-1)}$ and $\left\|E\left(\Delta_{m}\right)\right\|=\sqrt{2 m-1}$. Note that, here, the values $\left\|E_{12}\left(\Delta_{m}\right)\right\|$ and $\left\|E\left(\Delta_{m}\right)\right\|$ have the same order of growth for $m \rightarrow \infty$. Note also that $E_{10}(\Delta)=0$ for every closed segment $\Delta \subset[-1 ; 1] \backslash\{0\}$.

Definition 6.22. If, for (6.3) and (6.65), a relation between a J-orth.sp.f. $E_{\curlywedge}$ satisfying (6.1) and a space $\mathcal{\partial}-\widetilde{L}_{\vec{\sigma}}^{2}(\mathcal{\varepsilon})$ is given by $(6.70)$, then $\mathcal{\partial}-\widetilde{L}_{\vec{\sigma}}^{2}(\mathcal{E})$ is said to be a basic model space for $E_{\lambda}$ (compatible with (6.3), (6.15), and (6.66)) and the operator $W$ is said to be an operator of similarity corresponding to this space.

Note that Definition 6.22 does not contradict Definition 6.6, but amplifies it; if, for $J$ orth.sp.f. $E_{\lambda},(6.2)$ is fulfilled, then $\partial=I$, so the canonical scalar product is uniquely defined on $\mathscr{t}_{2}$ and coincides with $[\cdot, \cdot]$.

Now, we show that every space $\partial-\widetilde{L}_{\vec{\sigma}}^{2}(\mathcal{\varepsilon})$ can be considered as a basic model space for a suitable $J$-orth.sp.f. $E_{\lambda} \in D_{k}^{+}$.

Theorem 6.23. Let a space $\partial-\widetilde{L}_{\vec{\sigma}}^{2}(\mathcal{\varepsilon})$ be the expansion of a standard Krein space $\partial-L_{\vec{\sigma}}^{2}(\mathcal{E})$ generated by a collection $\left\{\tilde{g}_{j}(t)\right\}_{j=1}^{k}$ satisfying (6.16). Then there exist a Krein space $\mathscr{d}$ and a J-orth.sp.f. $E_{\lambda}$ on $d$ such that $E_{\lambda} \in D_{k}^{+}$and $2-\widetilde{L}_{\vec{\sigma}}^{2}(\mathcal{E})$ is a basic model space for $E_{\lambda}$.

Proof. Let $\mathscr{H}$ be the set of triples $(u, v, f(t))$ with $u, v \in \mathbb{C}^{k}$ and $f(t) \in \mathcal{\partial}-L_{\vec{\sigma}}^{2}(\mathcal{\varepsilon})$. For $x_{1}=$ $\left(u_{1}, v_{1}, f_{1}(t)\right)$ and $x_{2}=\left(u_{2}, v_{2}, f_{2}(t)\right)$, put (see (4.3))

$$
\begin{aligned}
& \left(x_{1}, x_{2}\right)=\left(u_{1}, u_{2}\right)_{\mathbb{C}^{k}}+\left(v_{1}, v_{2}\right)_{\mathbb{C}^{k}}+\left(f_{1}(t), f_{2}(t)\right)_{L_{\bar{\sigma}}^{2}(\varepsilon)^{\prime}} \\
& {\left[x_{1}, x_{2}\right]=\left(u_{1}, v_{2}\right)_{\mathbb{C}^{k}}+\left(v_{1}, u_{2}\right)_{\mathbb{C}^{k}}+\left[f_{1}(t), f_{2}(t)\right]_{L_{\bar{\sigma}}^{2}(\varepsilon)}}
\end{aligned}
$$

Next, let $\left\{e_{j}\right\}_{j=1}^{k}$ be the canonical basis in $\mathbb{C}^{k}$. For the intervals $\Delta=[\alpha, \beta)$, where $0 \notin \Delta$ and $\alpha \neq 0$, we put

$$
\begin{array}{r}
E(\Delta)\left(e_{j}, 0,0\right)=\left(0, \sum_{m=1}^{k}\left[\chi_{\Delta}(t) \tilde{g}_{j}(t), \chi_{\Delta}(t) \tilde{g}_{m}(t)\right]_{L_{\tilde{\sigma}}^{2}(\varepsilon)} \cdot e_{m}, \chi_{\Delta}(t) \tilde{g}_{j}(t)\right) \\
\text { for } j=1,2, \ldots, k, E(\Delta)(0, v, 0)=(0,0,0) \text { for every } v \in \mathbb{C}^{k}, \\
E(\Delta)(0,0, f(t))=\left(0, \sum_{m=1}^{k}\left[\chi_{\Delta}(t) f(t), \chi_{\Delta}(t) \tilde{g}_{m}(t)\right]_{L_{\tilde{\sigma}}^{2}(\varepsilon)} \cdot e_{m}, \chi_{\Delta}(t) f(t)\right) .
\end{array}
$$

Direct verification shows that $E(\Delta)$ generates a $J$-orth.sp.f. satisfying (6.1). The rest of this proof looks like the corresponding part of the proof of Theorem 6.7 with some evident modifications.

Next, the basic model space is constructed not only via the $J$-orth.sp.f. $E_{\lambda}$ itself and via the subspaces $\widetilde{\mathscr{C}}$ and $\mathscr{l}_{1}$ naturally generated by $E_{\mathcal{\lambda}}$ (note that, in this case, it is convenient to consider the mentioned subspaces as linear topological spaces), but also via some other subspaces and via the Hilbert structure introduced on $\widetilde{\mathscr{H}}$ and $\mathscr{H}_{1}$ that can be defined with some ambiguity. 
Proposition 6.24. Let a space $2-\widetilde{L}_{\vec{\sigma}}^{2}(\mathcal{E})$ (i.e., the expansion of standard Krein space $2-L_{\vec{\sigma}}^{2}(\mathcal{E})$ generated by a collection $\left\{\tilde{g}_{j}(t)\right\}_{j=1}^{k}$ with (6.16)) be a basic model space for a J-orth.sp.f. E $E_{\lambda}$ compatible with (6.3), (6.15), and (6.66). Then for every different decomposition

$$
\widetilde{H}=\mathscr{H}_{1} \dot{+} \mathscr{\ell}_{2}^{\prime}, \quad \mathscr{H}_{2}^{\prime}=\mathfrak{L}_{+}[\dot{+}] \mathfrak{L}_{-},
$$

where $\mathfrak{L}_{+}$and $\mathfrak{L}_{-}$are, respectively, uniformly positive and uniformly negative subspace, and $\mathfrak{L}_{1}+\mathfrak{L}_{+}$and $\mathscr{H}_{1}+\mathfrak{L}_{-}$are $E_{\lambda}$-invariant, there is a collection $\left\{\breve{g}_{j}(t)\right\}_{j=1}^{k}$ with (6.16) such that the expansion $\partial-\breve{L}_{\vec{\sigma}}^{2}(\mathcal{E})$ of $\partial-L_{\vec{\sigma}}^{2}(\varepsilon)$ generated by $\left\{\breve{g}_{j}(t)\right\}_{j=1}^{k}$ is also a basic model space for $E_{\lambda}$ compatible with (6.3), (6.15), and (6.73).

Proof. Note that a collection of unbounded elements $\left\{\breve{g}_{j}(t)\right\}_{j=1}^{k}$ is uniquely defined by a set composed by the following components: a choice of a normalized basis in $\mathscr{\ell}_{1}$, a decomposition $\widetilde{\mathscr{H}}=\mathscr{H}_{1}+\mathscr{\ell}_{2}^{\prime}$, and an isometric $J$-isometric map $W_{2}^{\prime}: \mathcal{\partial}-L_{\vec{\sigma}}^{2}(\varepsilon) \mapsto \mathscr{H}_{2}^{\prime}$. This collection always exists for the mentioned set, so it is enough to show the existence of $W_{2}^{\prime}$. Consider two cases.

(1) Let $\mathscr{\ell}_{2}^{\prime}=\mathscr{\ell}_{2}$. Thus, on the Krein space $\mathscr{\ell}_{2}$, there are two different $P_{2} E_{\lambda}$-invariant canonical decompositions. Then, thanks to Proposition 3.8 and (6.70)

$$
\left.P_{2} E_{\lambda}\right|_{\mathscr{L}_{2}}=D_{\left\{\mathscr{\ell}_{2}^{+}, \mathscr{L}_{2}^{-}\right\},\left\{\mathfrak{L}_{+}, \mathfrak{L}_{-}\right\}} W_{2} \mathrm{X}_{\mathcal{\lambda}} W_{2}^{-1}\left(D_{\left\{\mathscr{\ell}_{2}^{+}, \mathfrak{L}_{2}^{-}\right\},\left\{\mathfrak{L}_{+}, \mathfrak{L}_{-}\right\}}\right)^{-1}
$$

so one can take $W_{2}^{\prime}=D_{\left\{\mathscr{H}_{2}^{+}, \mathfrak{H}_{2}^{-}\right\},\left\{\mathfrak{L}_{+}, \mathfrak{L}_{-}\right\}} W_{2}$.

(2) Let $\mathscr{L}_{2}^{\prime} \neq \mathscr{\ell}_{2}$ and let the pairs $\left\{\mathscr{\ell}_{2}^{+}, \mathscr{L}_{2}^{-}\right\}$and $\left\{\mathfrak{L}_{+}, \mathfrak{L}_{-}\right\}$be related by a condition of the type (3.9). Then by Remark 3.6 and Proposition 3.7, one can take $W_{2}^{\prime}=\mathcal{M}_{\widetilde{\mathscr{\ell}}, \mathfrak{l}_{2}, \mathfrak{H}_{2}^{\prime}} \cdot W_{2}$.

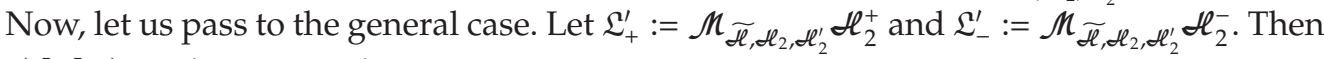
$\mathscr{H}_{2}^{\prime}=\mathfrak{L}_{+}^{\prime}[+] \mathfrak{L}_{-}^{\prime}$, and one can take

$$
W_{2}^{\prime}=D_{\left\{\mathfrak{L}_{+}^{\prime}, \mathfrak{L}_{-}^{\prime}\right\},\left\{\mathfrak{L}_{+}, \mathfrak{L}_{-}\right\}} \boldsymbol{M}_{\widetilde{\mathscr{L}}, \mathfrak{d}_{2}, \mathfrak{d}_{2}^{\prime}} \cdot W_{2}
$$

Corollary 6.25. Assume that $E_{\mathcal{\lambda}}$ is a J-orth.sp.f. satisfying (6.1), (6.3) is fixed, and $\partial-L_{\vec{\sigma}}^{2}(\varepsilon)$ is a standard Krein space. Then, independently of any realization of $(6.73)$, an expansion of $\partial-L_{\vec{\sigma}}^{2}(\varepsilon)$, that is a basic model space for $E_{\lambda}$ compatible with (6.73), exists if and only if there is a J-isometric operator $W_{2}: \partial-L_{\vec{\sigma}}^{2}(\varepsilon) \mapsto \mathscr{\ell}_{2}$ such that

$$
\left.P_{2} E_{\lambda}\right|_{\mathscr{L}_{2}}=W_{2} X_{\mathcal{\lambda}} W_{2}^{-1}
$$

Remark 6.26 (cf., with Proposition 6.8). Let $\stackrel{*}{\mathscr{\ell}}$ be the factor-space of $\mathscr{H}$ generated by $\mathscr{\ell}_{1}$. Then $\stackrel{*}{\mathscr{H}}$ is a Krein space and $\widetilde{E}_{\mathcal{\lambda}}$ induces, on this space, the $J$-orth.sp.f. $\stackrel{E}{\lambda}$. Note that the unique

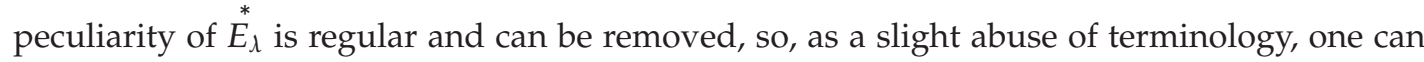

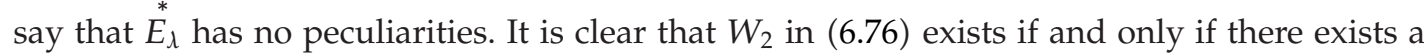
$J$-isometric operator $\stackrel{*}{W}: \partial-L_{\vec{\sigma}}^{2}(\varepsilon) \mapsto \stackrel{*}{\mathscr{\ell}}$ such that $\stackrel{*}{E_{\lambda}}=\stackrel{*}{W} \mathrm{X}_{\mathcal{\Lambda}} \stackrel{*}{W}{ }^{-1}$. Taking this reasoning and Corollary 6.25 into account, one can say that an arbitrariness in the choice of a basic model space for $J$-orth.sp.f. $E_{\mathcal{\lambda}}$ satisfying (6.1) does not depend on the choice of $P_{2}$ but essentially 
depends on an arbitrariness in the choice of a basic model space for $J$-orth.sp.f. $\stackrel{E}{\ell}_{\mathcal{*}}$ with the empty set of peculiarities. The last problem was considered in Section 4.

Proposition 6.27. Assume that spaces $2-\widetilde{L}_{\vec{\sigma}}^{2}(\varepsilon)$ and $\partial^{\prime}-\breve{L}_{\vec{\sigma}}^{2}(\varepsilon)$ are constructed on the base of the same space $L_{\vec{\sigma}}^{2}(\mathcal{E})$ passing by the following way: first, introduce on it two (maybe different) sesquilinear forms by fundamental symmetries 2 and 2 '; second, expand these two standard Krein spaces using collections of unbounded elements $\left\{\widetilde{g}_{j}(t)\right\}_{j=1}^{k}$ and $\left\{\breve{g}_{j}(t)\right\}_{j=1}^{k}$, respectively. Assume also that, first, $\partial-\widetilde{L}_{\vec{\sigma}}^{2}(\mathcal{E})$ and $\partial^{\prime}-\breve{L}_{\vec{\sigma}}^{2}(\varepsilon)$ are basic model spaces for a J-orth.sp.f. $E_{\lambda}$, second, $W^{(1)}$ and $W^{(2)}$ are corresponding operators of similarity, and, third, every function $f(t) \in L_{\vec{\sigma}}^{2}(\varepsilon)$ satisfies (6.34). Then $\partial=\partial^{\prime}$ and the spaces $\partial-\widetilde{L}_{\vec{\sigma}}^{2}(\varepsilon)$, and $\partial^{\prime}-\breve{L}_{\vec{\sigma}}^{2}(\varepsilon)$ coincide as subsets in $M_{\vec{\sigma}}(\varepsilon)$.

Proof. Condition (6.34) implies that $\left[W^{(1)} f(t), W^{(1)} f(t)\right]=\left[W^{(2)} f(t), W^{(2)} f(t)\right]$ is true for every $f(t) \in L_{\vec{\sigma}}^{2}(\varepsilon)$, so $[f(t), f(t)]_{2-\tilde{L}_{\vec{\sigma}}^{2}(\varepsilon)}=[f(t), f(t)]_{2-L_{\bar{\sigma}}^{2}(\varepsilon)}$. Hence $\mathcal{\partial}=\partial^{\prime}$. The rest of the reasoning is similar to that of Proposition 6.9.

Comparing the last proposition with Proposition 6.10, note that the fulfilment of (6.34) is essential for the simultaneous coincidence $\partial-\widetilde{L}_{\vec{\sigma}}^{2}(\mathcal{\varepsilon})$ with $\partial^{\prime}-\breve{L}_{\vec{\sigma}}^{2}(\varepsilon)$ as subsets in $M_{\vec{\sigma}}(\varepsilon)$, and $\partial-L_{\vec{\sigma}}^{2}(\mathcal{\varepsilon})$ with $\partial^{\prime}-L_{\vec{\sigma}}^{2}(\mathcal{\varepsilon})$ as standard Krein spaces, but at the same time is not necessary.

Theorem 6.28. Assume that spaces $\partial-\widetilde{L}_{\vec{\sigma}}^{2}(\mathcal{\varepsilon})$ and $\partial^{\prime}-\breve{L}_{\vec{\sigma}}^{2}(\mathcal{\varepsilon})$ are constructed on the base of the same space $L_{\vec{\sigma}}^{2}(\mathcal{E})$ passing by the following way: first, introduce on it two (maybe different) sesquilinear forms by fundamental symmetries 2 and $\partial^{\prime}$, and second, expand these two standard Krein spaces using collections of unbounded elements $\left\{\widetilde{g}_{j}(t)\right\}_{j=1}^{k}$ and $\left\{\breve{g}_{j}(t)\right\}_{j=1}^{k}$, respectively. Then $\partial-\widetilde{L}_{\vec{\sigma}}^{2}(\mathcal{E})$ and $\partial^{\prime}-\breve{L}_{\vec{\sigma}}^{2}(\mathcal{\varepsilon})$ are basic model spaces for a common J-orth.sp.f. $E_{\lambda}$ satisfying (6.1) if and only if there exist operator functions $U_{t}$ and $M_{t}, t \in[-1 ; 1]$, with (3.24) and (4.7), respectively, such that the sets $\widetilde{L}_{\vec{\sigma}}^{2}(\varepsilon)$ and $\left\{M_{t} U_{t} \tilde{f}(t)\right\}_{f(t) \in \tilde{L}_{\bar{\sigma}}^{2}(\varepsilon)}$ coincide as subsets of $M_{\vec{\sigma}}(\varepsilon)$.

The proof of this theorem is similar to that of Theorem 6.14 .

\subsection{The passage to J-symmetric operator families}

Up to this point, we discussed model representations not for a family $\mathfrak{Y} \in D_{\mathcal{K}}^{+}$but for a $J$-orth.sp.f. $E_{\curlywedge}$ with the unique spectral peculiarity in zero subjected to the condition

There exists $\mathcal{L}_{+} \in \mathfrak{M}^{+}(\mathscr{L}) \cap h^{+}$such that for every $\lambda \in \mathbb{R} \backslash\{0\}$ it is true $E_{\lambda} \mathfrak{L}_{+} \subset \mathfrak{L}_{+}$and for every closed interval $\Delta \subset \mathbb{R} \backslash\{0\}$ there is the decomposition $E(\Delta) \mathscr{\ell}=E(\Delta) \mathfrak{L}_{+}[+] E(\Delta) \mathfrak{L}_{-}$.

Now, let $\mathfrak{Y} \in D_{\mathcal{\kappa}}^{+}$be a $J$-symmetric commutative operator family. Then its linear span contains a family (not uniquely defined) $\mathfrak{Y}_{r}$ of $J$-s.a. operators such that the linear span of $\mathfrak{Y}_{r}$ coincides with the linear span of $\mathfrak{Y}$. In [20], it was proved that there is the decomposition $\mathscr{H}=\mathscr{H}_{r e}[\dot{+}] \mathscr{H}_{i m}$, where $\mathscr{H}_{r e}$ and $\mathscr{H}_{i m}$ are $\mathfrak{Y}$-invariant, all operators from $\left.\mathfrak{Y}_{r}\right|_{\mathscr{d}_{r e}}$ have real spectra, $\operatorname{dim} \mathscr{L}_{i m}<\infty$, and, at least, one $J$-s.a. operator from Alg $\left.\mathfrak{Y}\right|_{\mathscr{l}_{i m}}$ has no real points in its spectrum. Let $E_{\curlywedge}$ be an e.s.f. for $\left.\mathfrak{Y}_{r}\right|_{\mathscr{d}_{r e}}$. Extend the action of $E_{\lambda}$ for all $\mathscr{d}$ setting $\left.E_{\lambda}\right|_{\mathscr{d}_{i m}}=0$. This extended function $E_{\lambda}$ is said to be an e.s.f. of $\mathfrak{Y}$. Comparing Theorem 3.11 and Theorem 6.19, it is easy to obtain the following result. 
Theorem 6.29. If $\mathfrak{Y} \in D_{\kappa}^{+}$is a commutative J-symmetric family, its e.s.f. $E_{\curlywedge}$ satisfies (6.1), a canonical scalar product on th is compatible with (6.66), $2-\widetilde{L}_{\vec{\sigma}}^{2}(\mathcal{\varepsilon})$ is a basic model space for $E_{\lambda}$, and $W$ is a corresponding operator of similarity, then, for every operator $A \in \mathfrak{Y}$, there is a function $\varphi(t)$ such that

$$
\tilde{A}^{\uparrow}=W^{\uparrow} \cdot \Phi \cdot\left(W^{\uparrow}\right)^{-1}, \quad \tilde{A}=W \cdot \overline{\Phi^{\#}} \cdot W^{-1},
$$

where $\tilde{A}^{\uparrow}:=\left.\left(P_{0} \oplus P_{1}\right) A\right|_{\widetilde{\mathbb{R}^{\top}}}$, the space $\widetilde{\mathscr{A l}^{\uparrow}}$ and the operator $W^{\uparrow}$ are defined via (6.15) and (6.70), $\Phi=\left\{\Phi, \partial-L_{\vec{\sigma}}^{2}(\varepsilon)\right\}$, and $\bar{\Phi}$ is the multiplication operator by $\bar{\varphi}(t)$ also acting in the space $\partial-\widetilde{L}_{\vec{\sigma}}^{2}(\varepsilon)$.

Remark 6.30. If, under the conditions of Theorem 6.29, J-orth.sp.f. $E_{\lambda}$ satisfies (6.2), then the space $2-\widetilde{L}_{\vec{\sigma}}^{2}(\varepsilon)$ must be substituted by the space $\widetilde{L}_{\vec{\sigma}}^{2}(\mathcal{\varepsilon})$, and, respectively, the operator $\bar{\Phi}^{\#}$ must be substituted by the operator $\bar{\Phi}^{*}$.

Definition 6.31. If $\mathfrak{Y} \in D_{\kappa}^{+}$is a commutative $J$-symmetric family and $E_{\lambda}$ (satisfying (6.1)) is its e.s.f., then a basic model space for $E_{\curlywedge}$ is said to be also a basic model space for $\mathfrak{Y}$.

Remark 6.32. Assume that $\mathfrak{Y} \in D_{\kappa}^{+}$is a commutative $J$-symmetric family, its e.s.f. $E_{\curlywedge}$ satisfies (6.1), and $2-\widetilde{L}_{\vec{\sigma}}^{2}(\mathcal{E})$ and $W$ are, respectively, a basic model space and a corresponding operator of similarity for $\mathfrak{Y}$. If, under these conditions, an operator $A \in \mathfrak{Y}$ and a function $\varphi(t)$ are related by (6.78), then, according to Definition 3.12, the function $\varphi(t)$ is a portrait of the operator $A$ (with respect to $E_{\curlywedge}$ ), and the operator $A$ is an original (not unique in the general case) of $\varphi(t)$ in $\mathfrak{Y}$. It is clear that the portrait of an operator does not depend on the choice of the basic model space $2-\widetilde{L}_{\vec{\sigma}}^{2}(\mathcal{E})$ and can be found via (3.15)(c), but at the same time depends on the choice of $E_{\lambda}$.

Remark 6.33. Theorem 6.29 brings a natural problem concerning a characterization of functions that can be portraits for operators from a given commutative $J$-symmetric operator family $\mathfrak{Y} \in D_{\kappa}^{+}$with a fixed choice of $J$-orth.sp.f. $E_{\lambda}$. A partial answer to the problem is contained by Propositions 5.4 and 5.5. Indeed, under the present conditions, the function $v(t)$ from (5.5) has the form

$$
v(t)=\left\{\begin{array}{ll}
\int_{-1}^{t} G(t) d \sigma(t), & t \in[-1 ; 0), \\
-\int_{t}^{1} G(t) d \sigma(t), & t \in(0 ; 1],
\end{array} \quad \text { where } G(t)=1+\sum_{j=1}^{k}\left\|\widetilde{g}_{j}(t)\right\|_{\varepsilon}^{2} .\right.
$$

\section{Closing remarks}

Spectral functions are a traditional source for constructions of model spaces for different classes of normal operators in Hilbert spaces, so it is natural to use the same approach in Krein spaces. The first theorem concerning the existence of spectral functions for $\pi$-s.a. operators was published by Krěn and Langer in [28] (see also [6] for detailed proofs). The spectral functions introduced in Section 3.2 are a particular case of generalized spectral functions [1]. Our definition is inspired by spectral measures arising in the operator theory in indefinite metric spaces (see $[3,6,8,29]$ ) but formally, independent (see [30]) of this Theory. Another development related with generalized spectral functions can be found in [31-34]. There are some works on model representations for self-adjoint operators and algebras in Pontryagin 
spaces (the majority of them consider the case with the rank of indefiniteness 1) [35-40], (see also [41] for more references). In [12], the case of a self-adjoint cyclic operator acting in an arbitrary Pontryagin space was considered. The class $D_{\kappa}^{+}$that is investigated in the present paper differs from the well-known class of definitizable operators (see [42] for discussion) and represents another natural generalization of the class of self-adjoint operators in Pontryagin spaces. The notion of unbounded elements was given by the author in [43], thereupon, this notion was used in [9], where the existence of a basic model space for a single $\pi$-self-adjoint operator was proved. The latter result was applied in [10] (devoted to a description of a broad functional calculus for $\pi$-self-adjoint operators) and [11], where the bicommutant problem for a $\pi$-self-adjoint operator was studied. Some remarks concerning a model representation of $\pi$ unitary operators were given in [44]. A connection between the spaces of the type $L_{\sigma}^{\infty} \cap L_{v}^{2}$ and operator algebras was pointed out for the first time in [45] (for Pontryagin spaces with the rank of indefiniteness 1) and [46]. On this subject, see also [47, 48]. Although the concept of basic model spaces for commutative families of $\pi$-self-adjoint or $J$-self-adjoint operators of the $D_{\kappa}^{+}$-class was used by the author in some previous papers, its complete description and corresponding proofs as well as the discussion of the ambiguity of basic model spaces are given here for the first time.

\section{Acknowledgments}

This work was completed with the support of Project FONACIT (Venezuela) G-97000668. The author is very grateful to Tom Berry and Luís Mata Lorenzo for their assistance in making this paper more readable. The author would like also to thank the referee of this paper for his/her very useful comments.

\section{References}

[1] I. Colojoară and C. Foiaş, Theory of Generalized Spectral Operators, Gordon and Breach, New York, NY, USA, 1968.

[2] V. Strauss, "The structure of a family of commuting J-selfadjoint operators," Ukrainskiu Matematicheskiu Zhurnal, vol. 41, pp. 1431-1433, 1989 (Russian), English translation in Ukrainian Mathematical Journal, vol. 41, no. 10, pp. 1232-1234, 1989.

[3] J. Bognár, Indefinite Inner Product Spaces, Springer, New York, NY, USA, 1974.

[4] T. Ya. Azizov and I. S. Iohvidov, "Linear operators in spaces with an indefinite metric and their applications," in Mathematical Analysis, vol. 17 of Itogi Nauki i Tekhniki, pp. 113-205, Vserossiisky Institut Nauchnoi i Tekhnicheskoi Informatsii, Moscow, Russia, 1979.

[5] I. S. Iohvidov, M. G. Krern, and H. Langer, Introduction to the Spectral Theory of Operators in Spaces with an Indefinite Metric, vol. 9 of Mathematical Research, Akademie, Berlin, Germany, 1982.

[6] H. Langer, "Spectral functions of definitizable operators in KreIn spaces," in Functional Analysis (Dubrovnik, Yugoslavia, 1981), vol. 948 of Lecture Notes in Mathematics, pp. 1-46, Springer, Berlin, Germany, 1982.

[7] I. Gohberg, P. Lancaster, and L. Rodman, Indefinite Linear Algebra and Applications, Birkhäuser, Basel, Switzerland, 2005.

[8] T. Ya. Azizov and I. S. Iokhvidov, Foundations of the Theory of Linear Operators in Spaces with Indefinite Metric, Nauka, Moscow, Russia, 1986.

[9] V. Strauss, "A model representation for a simplest $\pi$-selfadjoint operator," in Collection: Funktsionalnii Analiz, Spectral Theory, pp. 123-133, State Pedagogical Institute of Uliyanovsk, Ulyanovsk, Russia, 1984. 
[10] V. Strauss, "Functional representation of an algebra generated by a selfadjoint operator in a Pontryagin space," Funktsional'ny̆ Analiz i ego Prilozheniya, vol. 20, no. 1, pp. 91-92, 1986 (Russian), English translation: Functional Analysis and Applications.

[11] V. Strauss, "Functional representation of operators that doubly commute with a selfadjoint operator in a Pontryagin space," Sibirskiu Matematicheskiu Zhurnal, vol. 29, no. 6, pp. 176-184, 1988 (Russian), English translation: Siberian Mathematical Journal, vol. 29, no. 6, pp. 1012-1018, 1988.

[12] P. Jonas, H. Langer, and B. Textorius, "Models and unitary equivalence of cyclic selfadjoint operators in Pontrjagin spaces," in Operator Theory and Complex Analysis (Sapporo, Japan, 1991), vol. 59 of Operator Theory: Advances and Applications, pp. 252-284, Birkhäuser, Basel, Switzerland, 1992.

[13] I. Gohberg and B. Reichstein, "On classification of normal matrices in an indefinite scalar product," Integral Equations and Operator Theory, vol. 13, no. 3, pp. 364-394, 1990.

[14] O. V. Holtz and V. Strauss, "Classification of normal operators in spaces with indefinite scalar product of rank 2," in Proceedings of the 4th Conference of the International Linear Algebra Society, vol. 241-243 of Linear Algebra and Its Applications, pp. 455-517, Rotterdam, The Netherlands, 1996.

[15] T. Ya. Azizov and V. Strauss, "Spectral decompositions for special classes of self-adjoint and normal operators on Krein spaces," in Spectral Analysis and Its Applications, vol. 2 of Theta Series in Advanced Mathematics, pp. 45-67, Theta, Bucharest, Romania, 2003.

[16] A. Gheondea, "Pseudo-regular spectral functions in Krĕn spaces," Journal of Operator Theory, vol. 12, no. 2, pp. 349-358, 1984.

[17] N. Dunford and J. T. Schwartz, Linear Operators. I. General Theory, Interscience, New York, NY, USA, 1958.

[18] E. Kissin and V. Shulman, Representations on Krein Spaces and Derivations of C*-Algebras, vol. 89 of Pitman Monographs and Surveys in Pure and Applied Mathematics, Longman, Harlow, UK, 1997.

[19] N. Dunford and J. T. Schwartz, Linear Operators-Part III: Spectral Operators, John Wiley \& Sons, New York, NY, USA, 1971.

[20] T. Ya. Azizov and V. Strauss, "On a spectral decomposition of a commutative family of operators on spaces with indefinite metric," Methods of Functional Analysis and Topology, vol. 11, no. 1, pp. 10-20, 2005.

[21] S. G. KreIn, Y. I. Petunin, and E. M. Semënov, Interpolyatsiya Lineinykh Operatorov, Nauka, Moscow, Russia, 1978.

[22] M. A. Naĭmark, Normed Algebras, Wolters-Noordhoff, Groningen, The Netherlands, 3rd edition, 1972.

[23] M. Š. Birman and M. Z. Solomjak, Spectral Theory of Self-Adjoint Operators in Hilbert Space, Leningrad University, Leningrad, Russia, 1980.

[24] O. Bratteli and D. W. Robinson, Operator Algebras and Quantum Statistical Mechanics, vol. 1, Springer, New York, NY, USA, 1979.

[25] M. Reed and B. Simon, Methods of Modern Mathematical Physics. I: Functional Analysis, Academic Press, New York, NY, USA, 2nd edition, 1980.

[26] N. I. Akhiezer and I. M. Glazman, Theory of Linear Operators in Hilbert Space, Monographs and Studies in Mathematics, Pitman, London, UK, 1981.

[27] D. A. Suprunenko and R. I. Tyškevič, Perestanovochnye Matritsy, Nauka i Tehnika, Minsk, Russia, 1966.

[28] M. G. Kreln and G. K. Langer, "On the spectral function of a self-adjoint operator in a space with indefinite metric," Doklady Akademii Nauk SSSR, vol. 152, pp. 39-42, 1963 (Russian).

[29] P. Jonas, "On the functional calculus and the spectral function for definitizable operators in KreIn space," Beiträge zur Analysis, no. 16, pp. 121-135, 1981.

[30] V. Strauss, "Integro-polynomial representation of regular functions of an operator whose spectral function has critical points," Akademiya Nauk Ukrainskŏ SSR. Doklady, Seriya A, vol. 8, no. 85, pp. 26-29, 1986 (Russian).

[31] I. Bacalu, "S-decomposable operators in Banach spaces," Revue Roumaine de Mathématiques Pures et Appliquées, vol. 20, no. 10, pp. 1101-1107, 1975.

[32] V. E. Lyantze, "On one generalization of the notion of spectral measure," Matematicheskiı Sbornik, vol. 61, no. 1, pp. 80-120, 1983 (Russian).

[33] B. Nagy, "Operators with spectral singularities," Journal of Operator Theory, vol. 15, no. 2, pp. 307-325, 1986.

[34] B. Nagy, "Spectral measures with singularities," Acta Mathematica Hungarica, vol. 49, no. 1-2, pp. 51-64, 1987. 
[35] M. A. NaĬmark, "Commutative algebras of operators in a space $\Pi_{1}$, " Revue Roumaine de Mathématiques Pures et Appliquées, vol. 9, pp. 499-528, 1964 (Russian).

[36] A. I. Loginov, "Complete commutative symmetric operator algebras in a Pontrjagin space $\Pi_{1}$," Matematicheskiu Sbornik, vol. 84 (126), pp. 575-582, 1971 (Russian).

[37] V. S. Šul'man, "Symmetric Banach algebras of operators in a space of type $\Pi_{1}$," Matematicheskiu Sbornik. Novaya Seriya, vol. 89(131), pp. 264-279, 1972 (Russian).

[38] S. N. Litvinov, "Description of commutative symmetric algebras of operators in the Pontryagin space $\Pi_{1}, "$ Doklady Akademii Nauk UzSSR, no. 1, pp. 9-12, 1987 (Russian).

[39] H. Langer and B. Textorius, "L-resolvent matrices of symmetric linear relations with equal defect numbers; applications to canonical differential relations," Integral Equations and Operator Theory, vol. 5, no. 2, pp. 208-243, 1982.

[40] P. Jonas and H. Langer, "A model for $\pi$-selfadjoint operators in $\pi_{1}$-spaces and a special linear pencil," Integral Equations and Operator Theory, vol. 8, no. 1, pp. 13-35, 1985.

[41] M. A. Nămark and R. S. Ismagilov, "Representations of groups and algebras in spaces with indefinite metric," in Mathematical Analysis, pp. 73-105, Vserossiisky Institut Nauchnoi i Tekhnicheskoi Informatsii, Moscow, Russia, 1969.

[42] T. Ya. Azizov, L. I. Sukhocheva, and V. A. Shtraus, "Operators in a Kreŭn space," Matematicheskie Zametki, vol. 76, no. 3, pp. 324-334, 2004 (Russian).

[43] V. Strauss, "Some singularities of the spectral function of a $\pi$-selfadjoint operator," in Functional Analysis, No. 21, pp. 135-146, State Pedagogical Institute of Uliyanovsk, Uliyanovsk, Russia, 1983.

[44] V. Strauss, "On an analogue of the Wold decomposition for a $\pi$-semi-unitary operator and its model representation," in Harmonic Analysis and Operator Theory (Caracas, Venezuela, 1994), vol. 189 of Contemporary Mathematics, pp. 473-484, Proceedings of the American Mathematical Society, Providence, RI, USA, 1995.

[45] M. A. Naĭmark, "Commutative operator algebras in the space $\Pi_{1}$," Doklady Akademii Nauk SSSR, vol. 156, pp. 734-737, 1964 (Russian).

[46] H. Langer, "Spectraltheorie linearer Operatoren in J-räumen und enige Anwendungen auf die Shar $L(\lambda)=\lambda^{2} I+\lambda B+C$," Habilitationsschrift, Dresden University of Technology, Dresden, Germany, 1965.

[47] V. Strauss, "A functional description for the commutative $W J^{*}$-algebras of the $D_{\kappa}^{+}$-class," in Operator Theory and Indefinite Inner Product Spaces (Vienna, Austria, 2004), vol. 163 of Operator Theory: Advances and Applications, pp. 299-335, Birkhäuser, Basel, Switzerland, 2006.

[48] V. Strauss, "On models of function type for a special class of normal operators in Kreln spaces and their polar representation," Methods of Functional Analysis and Topology, vol. 13, no. 1, pp. 67-82, 2007. 


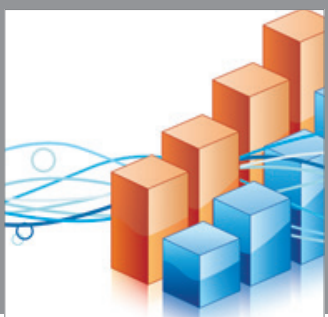

Advances in

Operations Research

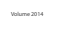

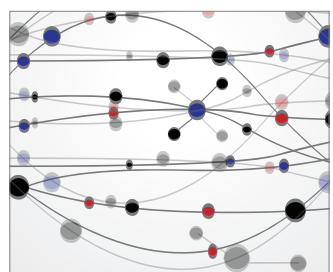

\section{The Scientific} World Journal
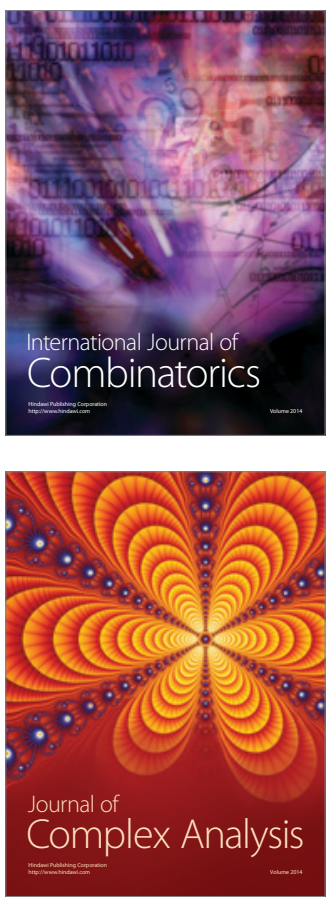

International Journal of

Mathematics and

Mathematical

Sciences
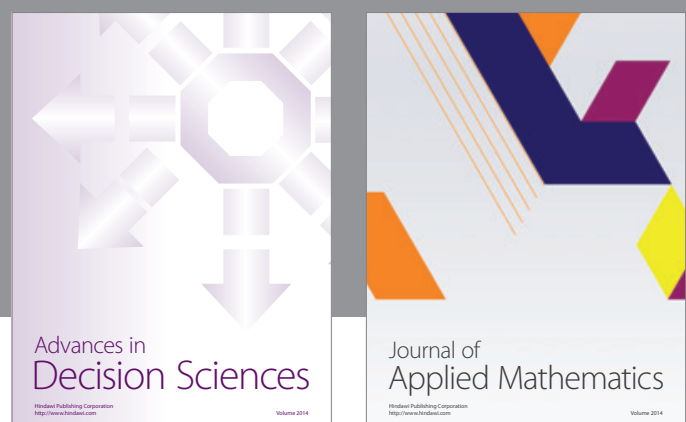

Journal of

Applied Mathematics
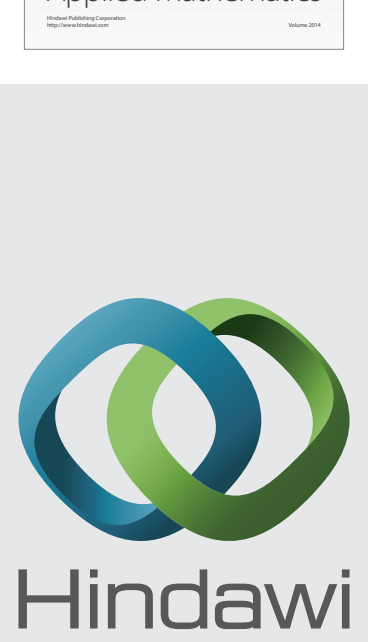

Submit your manuscripts at http://www.hindawi.com
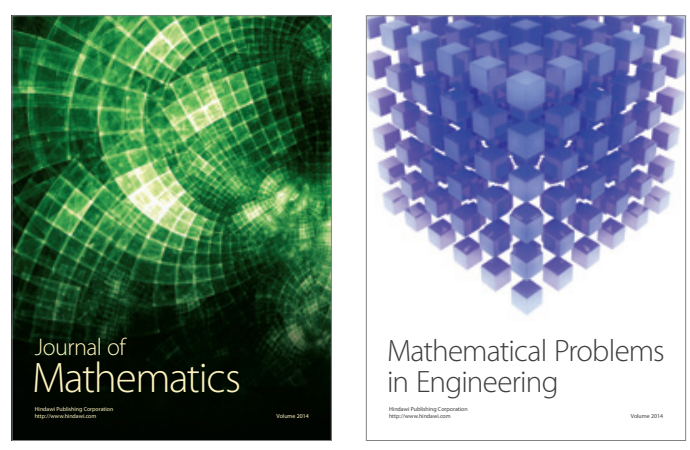

Mathematical Problems in Engineering
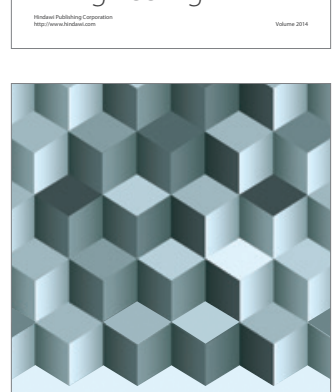

Journal of

Function Spaces
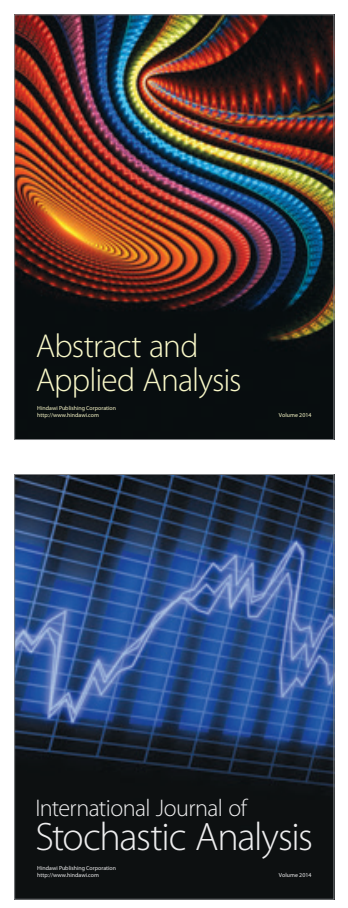

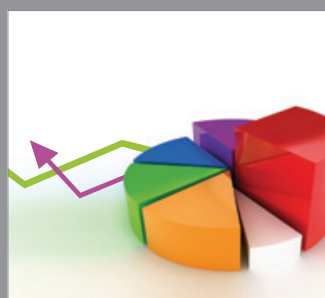

ournal of

Probability and Statistics

Promensencen
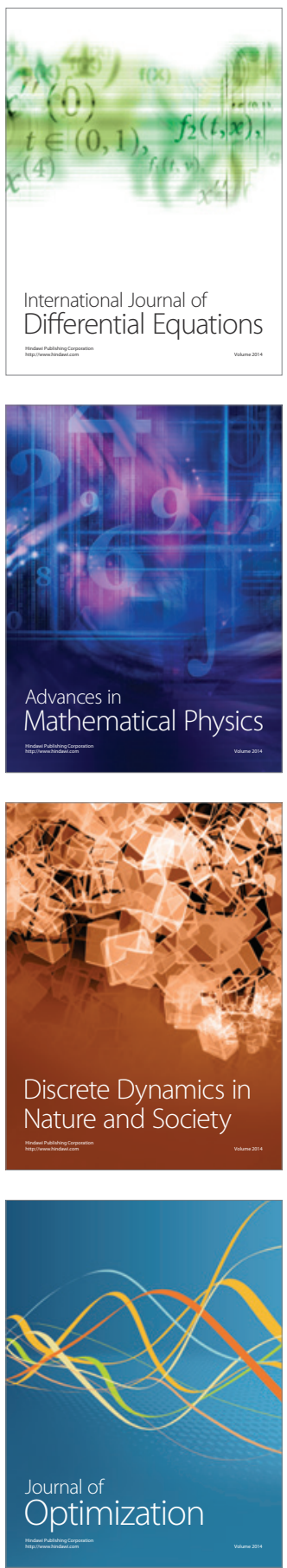